Experimental results on synchronization control of ship rendezvous operations

M. Wondergem

DCT.2004.30

Traineeship report $\quad: 8^{\text {th }}$ March 2004

Supervisor NTNU : prof. dr. K. Y. Pettersen

Supervisor TU/e : prof. dr. H. Nijmeijer

Advisor NTNU : siv. ing. E. Kyrkjebø 


\section{Acknowledgements}

I am very grateful that prof.dr. Henk Nijmeijer and prof.dr. Kristin Y. Pettersen have given me the opportunity to do my second traineeship in Norway on the subject of synchronization control of ship rendezvous operations.

I like to thank prof.dr. Kristin Y. Pettersen for her hospitality, her enthusiasm, her advice and comments and all the other things she arranged for me during my stay in Norway. This applies also to siv.ing. Erik Kyrkjebø and siv.ing. Roger Skjetne, who helped me a lot during this project. Every question I had, I could ask to one of them. It has been a great pleasure to work in this group.

I like to thank prof.dr. Henk Nijmeijer for his very nice contribution on the CAMS article, which was maybe the finishing touch we needed.

I should thank all the people in my office as well. Espen, Andreas and Anne Marthine for their friendship and nice talks during the lunch and dinner. 


\section{Abstract}

This report presents experimental results on rendezvous control of ships using an external synchronization control scheme. In particular, a leader-follower synchronization scheme for underway replenishment of ships is verified through a back-to-back comparison between experimental results and ideal simulations. The experiments illustrate that the synchronization controller yields exponential convergence of the closed-loop errors for position keeping, and uniform ultimate boundedness of the closed-loop errors during trajectory tracking. The gain tuning process is motivated, and the effects of measurement noise and environmental disturbances on the control scheme performance are discussed. 


\section{Contents}

Acknowledgements $\quad 1$

Abstract $\quad 2$

Contents

Chapter 1 Introduction $\quad 5$

1.1 Scope of the assignment 5

$\begin{array}{lr}1.2 \text { Outline } & 6\end{array}$

Chapter 2 Kinematics and dynamics of ships 7

$\begin{array}{llr}2.1 & \text { Coordinate systems } & 7\end{array}$

2.2 Ship dynamics $r$

$\begin{array}{ll}\text { Chapter } 3 \text { The observer-controller scheme used for ship rendezvous operations } & 10\end{array}$

$\begin{array}{llr}3.1 & \text { Observer-controller scheme } & 10\end{array}$

3.2 Practical remarks on the proposed observer-controller scheme 12

$\begin{array}{ll}3.3 & \text { Summary of sufficient conditions } \\ \end{array}$

Chapter 4 The MClab: Simulation and experimental setup $\quad 15$

4.1 The MClab: equipment $\quad 15$

$\begin{array}{llr}4.2 & \text { Simulation and experimental setup } & 17\end{array}$

$\begin{array}{lr}\text { Chapter } 5 \text { Underway replenishment: position keeping } & 19\end{array}$

$\begin{array}{llr}5.1 & \text { Behaviour of ships } & 19\end{array}$

5.2 Influence of observer and controller gains on the performance of the scheme 21

5.3 Experimental results during position keeping: gain set $1 \quad 22$

5.4 Experimental results during position keeping: gain set $2 \quad 25$

$\begin{array}{lll}5.5 & \text { Performance during position keeping } & 28\end{array}$

Chapter 6 Underway replenishment: Trajectory tracking 31

6.1 Experimental results trajectory tracking gain set $1 \quad 31$

6.2 Experimental results trajectory tracking gain set $3 \quad 34$

$\begin{array}{lll}6.3 & \text { Performance during trajectory tracking } & 37\end{array}$

$\begin{array}{lr}\text { Conclusion and recommendations } & 40\end{array}$

$\begin{array}{ll}\text { Bibliography } & 41\end{array}$

Appendix A: Full scale replenishment, some background. $\quad 42$

Appendix B: Gain tuning procedure for observer-controller scheme. $\quad 44$

Appendix C: Screw and rudder models. $\quad 46$ 
Appendix D: List of errors, problems and possible solutions during the simulations and experiments at the MCLab.

$\begin{array}{ll}\text { Appendix E: IP address and password. } & 49\end{array}$

Appendix F: Influence of the different observer and controller gains. 50

Appendix G: Linearized observer-controller scheme. $\quad 51$

Appendix H: Figures position keeping gain set 1 and set $2 . \quad 52$

Appendix I: Figures trajectory tracking gain set 1 and set $3 . \quad 60$

Appendix J: Table performance tracking straight line 68

Appendix K: Overview of the simulations and experiments $\quad 69$

$\begin{array}{ll}\text { Appendix L: Conference article } & 74\end{array}$ 


\section{Chapter 1}

\section{Introduction}

\subsection{Scope of the assignment}

To avoid or shorten port time it is necessary for ships to do underway replenishment (UNREP) at sea. The primary aim of an UNREP operation is the safe delivery of the maximum amount of cargo and fuel from one ship to another ship in the minimum amount of time.

There are two methods to replenish a ship, vertical replenishment (VERTREP) and connected replenishment (CONREP). During VERTREP operations helicopters are used to transport the cargo from the supply vessel or tanker to the replenished ship and vice versa. The ships are in close proximity, or miles apart depending on the tactical situation for military purposes and economic aspects for civil purposes.

During connected replenishment (CONREP) operations, two ships steam side by side and hoses and lines are used to transfer fuel, cargo, ammunition or personnel from one ship to the other. In appendix A some background information is given about full scale replenishment operations.

An external synchronization scheme was recently proposed by Kyrkjebø and Pettersen (2003) to dynamically control the supply ship to the position and velocity of a desired trajectory created on basis of the actual position of the replenished ship during CONREP operations. The trajectory for the supply ship is only based on the actual position of the replenished ship, which means that the supply ship is controlled with respect to the actual position of the main ship. This in contradiction to schemes where the ships track each their own predefined trajectory to do a replenishment operation. The supply ship is then controlled to the position where the replenished ship is supposed to be, but not necessarily is.

Using the proposed external synchronization scheme the supply ship synchronizes itself to the actual position of the replenished ship. So the error between the reference trajectory of the replenished ship and the actual position of the replenished ship can easily be removed. The intention is that this will result in lower errors between the ships, which will increase the safety during CONREP operations.

Another big advantage of this scheme is that the observer only needs the dynamic model of the supply ship and the actual positions of the ships. This means that it is possible to replenish different ships with one supply vessel without any knowledge of the dynamical behaviour of the replenished ships. This makes underway replenishment more interesting for civil applications.

Further in this report the term "main ship" will be used instead of replenished ship and the term "supply ship" will be used for the replenishment ship.

Of course this scheme is not only suitable for underway replenishment operations, but also suitable for all purposes where there is one leader and one or more followers. The only restriction is that the dynamic model(s) of the follower(s) and position information of the leader and follower(s) is available. This means that this scheme can be used in all kind of formation control purposes, like trajectory tracking as well as position keeping.

The goal of this project is to experimentally verify the theoretical results of the observer controller scheme presented by Kyrkjebø and Pettersen (2003) during underway replenishment at sea. In particular, the experiments aim at obtaining an increased understanding of the proposed observer-controller scheme; as well as to get insight in its advantages and its shortcomings. 


\subsection{Outline}

In chapter 2 a short overview is given about the kinematics and dynamics in ship control. The different coordinate systems and the relations between them are discussed. In addition the dynamic model of the ship is given in the different coordinate systems and the properties of the dynamic model are discussed.

Chapter 3 presents the proposed observer-controller scheme and the idea of leader-follower synchronization is explained. The assumptions and the conditions among which stability and boundedness of the closed loop errors is guaranteed are given. Besides practical remarks are made about the use of this scheme during an underway replenishment operation at sea.

The keynote of chapter 4 is to give an overview of the experimental environment and present the simulation and experimental setup. An overview of the equipment available at the MClab is given. The implementation of the scheme, the task of the main ship and the interpretation of the simulation and experimental results are described.

The topic of chapter 5 is position keeping. Here the tuning of the scheme is covered in detail. The theoretical results are verified with the experimental results obtained using a back-to-back comparison with simulation results under ideal conditions. The problems with some set of gains are explained, how these problems are caused and how these problems are solved. Finally the performance of the observercontroller scheme using different sets of gains during position keeping is assessed.

The experimentally obtained results during trajectory tracking are presented in chapter 6 . Again the theoretic results are verified with the experimental obtained results using a back-to-back comparison with simulation results under ideal conditions. Furthermore the problems with some set of gains are explained and how they are soived. This chapter finishes with an overview of the performance of the scheme with different set of gains.

Finally, some conclusions are drawn and recommendations are given for further work. 


\section{Chapter 2}

\section{Kinematics and dynamics of ships}

This chapter gives a short overview of the kinematics and dynamics in ship control. In particular the kinematics and dynamics, which are used in this report will be discussed. In section 2.1 the different coordinate systems and the relations between them are discussed. The 3 DOF nonlinear manoeuvring model for surface vessels is given and discussed in section 2.2.

\section{$2.1 \quad$ Coordinate systems}

In this report two different coordinate systems are used. The body fixed coordinate system is used, which is a body fixed frame on the ship. While the NED (North East Down) coordinate system is a fixed coordinate system in space. In this case the NED frame is a fixed frame in the basin of the MClab. In Figure 2.1 the different coordinate systems are shown.

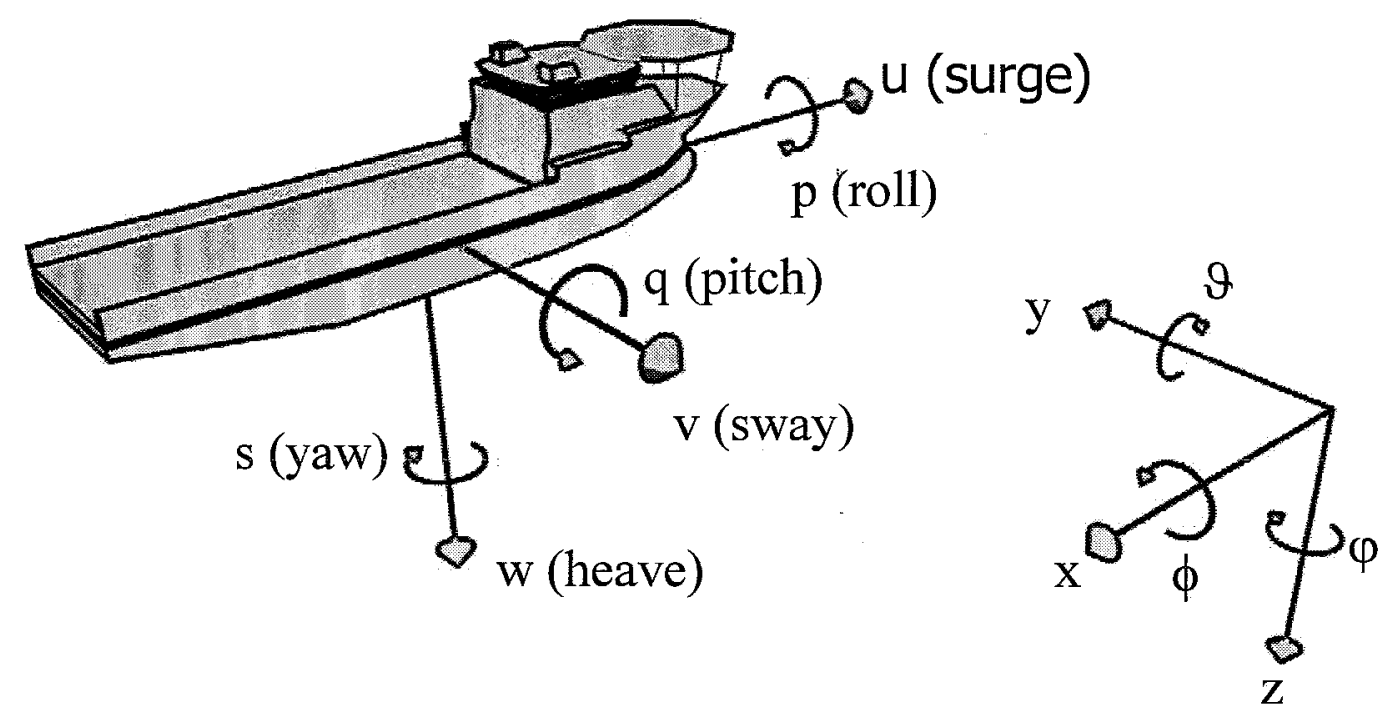

Figure 1.1 NED frame and body-fixed frame (by Andreas Lund Danielsen)

Here $p^{n}=\left[\begin{array}{lll}x & y & z\end{array}\right]^{T}$ represents the earth fixed position of the ship, while $\theta=\left[\begin{array}{lll}\phi & \vartheta & \varphi\end{array}\right]^{T}$ represents the attitude of the ship. The vector $v_{o}{ }^{b}=\left[\begin{array}{lll}u & v & w\end{array}\right]^{T}$ holds the body fixed linear velocities of the ship and $\omega_{o}{ }^{b}=\left[\begin{array}{lll}p & q & r\end{array}\right]^{T}$ the body fixed angular velocities of the ship. In this report the 3 DOF nonlinear manoeuvring model for surface vessels (Fossen, 2002) is used, which means that only the surge, sway and yaw direction of the ship are controlled.

$\eta=\left[\begin{array}{lll}x & y & \varphi\end{array}\right]^{T}$ and $v=\left[\begin{array}{lll}u & v & r\end{array}\right]^{T}$ 
The rotation matrix around the $z$-direction (2.3) defines the transformation from body fixed coordinates to the NED coordinates:

$\dot{\eta}=R(\varphi) v$

$R(\varphi)=\left[\begin{array}{ccc}\cos (\varphi) & -\sin (\varphi) & 0 \\ \sin (\varphi) & \cos (\varphi) & 0 \\ 0 & 0 & 1\end{array}\right]$

It is proved that $R(\varphi)^{-1}=R(\varphi)^{T}$ (Fossen, 2002), which results in the inverse transformation:

$v=R(\varphi)^{T} \dot{\eta}$

The time derivative of the rotation matrix can be calculated using the angular velocity matrix:

$$
\begin{aligned}
& \dot{R}(\varphi)=R(\varphi) S(r) \\
& S(r)=\left[\begin{array}{ccc}
0 & -r & 0 \\
r & 0 & 0 \\
0 & 0 & 0
\end{array}\right]
\end{aligned}
$$

\subsection{Ship dynamics}

In ship dynamics it is common to use the dynamics with respect to the body fixed frame, because then the entries of the matrices are independent of the position and heading of the ship. The dynamics of a surface ship can be described in the body fixed frame by using the 3 DOF nonlinear manoeuvring model for surface vessels (Fossen, 2002):

$$
M \dot{v}+C(v) v+D(v) v=\tau_{v}
$$

The matrix $M$ is the matrix of inertia and added mass. The $C$ matrix represents the Coriolis and Centripetal forces, but contains also added mass effects. The $D$ matrix contains the hydrodynamic damping coefficients. For a rigid body moving through an ideal fluid the $D$ matrix is non-symmetric and strictly positive (Fossen, 2002). This is motivated by energy dissipation. All marine vehicles/vessels dissipates energy thanks to the damping forces, $\tau=D v$, created by the ocean. This requires that the damping matrix should be strictly positive. The $\mathrm{C}$ matrix for a rigid body moving through an ideal fluid is skewsymmetric and can be calculated from the so-called Christoffel symbols (Fossen, 2002).

$$
\begin{aligned}
& x^{T} D(v) x>0 \text { for all } x \neq 0 \quad \forall \quad x \in R^{3} \quad v \in R^{3} \\
& C(v)=-C(v)^{T} \quad \forall \quad v \in R^{3}
\end{aligned}
$$

Using the relations (2.3) and (2.4) between the body fixed frame and NED frame, the dynamics in the NED frame can be derived.

$$
\dot{\eta}=R(\varphi) v \Leftrightarrow v=R(\varphi)^{T} \dot{\eta}
$$


$\ddot{\eta}=R(\varphi) \dot{v}+\dot{R}(\varphi) v \Leftrightarrow \dot{v}=R(\varphi)^{T}\left(\ddot{\eta}-\dot{R}(\varphi) R^{T}(\varphi) \dot{\eta}\right)$

$R(\varphi) M R(\varphi)^{T}\left(\ddot{\eta}-\dot{R}(\varphi) R^{T}(\varphi) \dot{\eta}\right)+R(\varphi) C(v) R(\varphi)^{T} \dot{\eta}+R(\varphi) D(v) R(\varphi)^{T} \dot{\eta}=R(\varphi) \tau_{v}$ (2.12)

Define:

$M^{*}(\eta)=R(\varphi) M R(\varphi)^{T}$

$C^{*}(v, \eta)=R(\varphi)(C(v)-M S(r)) R(\varphi)^{T}$

$D^{*}(v, \eta)=R(\varphi) D(v) R(\varphi)^{T}$

Using the notation (2.13) in (2.12) the dynamics in NED coordinates can be written as:

$M^{*}(\eta) \ddot{\eta}+C^{*}(v, \eta) \dot{\eta}+D^{*}(v, \eta) \dot{\eta}=\tau_{\eta}$

with the following properties (Fossen, 2002):

$M^{*}(\eta)=M^{*}(\eta)^{T} \quad \forall \quad \eta \in R^{3}$

$s^{T}\left[M^{*}(\eta)-2 C^{*}(v, \eta)\right] s=0 \quad \forall \quad s \in R^{3}, v \in R^{3}, \eta \in R^{3}$

$x^{T} D^{*}(v, \eta) x>0 \quad \forall x \in R^{3} \quad v \in R^{3}, \eta \in R^{3}$

It should be noticed that, in contrast to the dynamics in the body fixed frame, the entries of the matrices describing the dynamics in the NED frame are dependent of the position and heading of the ship. In chapter 5 it is seen that this is for tuning of the scheme a very important difference. 


\section{Chapter 3}

\section{The observer-controller scheme used for ship rendezvous operations}

In this chapter the external synchronization observer-controller scheme will be presented. In section 3.1 the idea of external synchronization is explained and the observer-controller synchronization scheme is given. The assumptions and the conditions to guarantee stability, convergence and boundedness of the synchronization system are given. In section 3.2 practical remarks are made about the use of this scheme during an underway replenishment operation at sea, while in section 3.3 the conditions to guarantee convergence and boundedness of the synchronization system are summarized.

\subsection{Observer-controller scheme}

The proposed synchronization scheme by Kyrkjebø and Pettersen (2003) is a leader-follower scheme, developed by Rodriguez-Angeles (2002) (Nijmeijer and Rodriguez-Angeles, 2003), which controls the supply ship to the desired position and desired velocity based on the actual state of the main ship. So basically the main ship creates a trajectory for the supply ship, which the supply ship is supposed to follow. It is assumed that only position measurements are available, the dynamic model of the supply ship is known and the supply ship is a fully actuated ship.

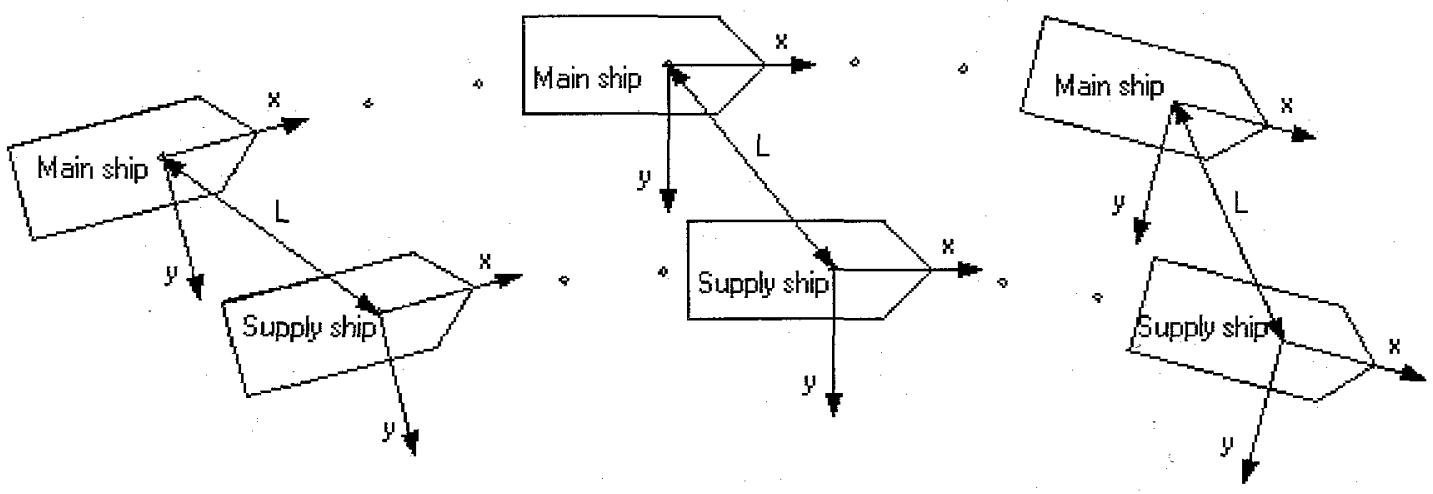

Figure 3.1 Trajectory main ship and desired trajectory supply ship.

In Figure 3.1 the idea of leader-follower synchronization is visualized. The main ship tracks a more or less arbitrarily path, while the supply ship moves at a predefined distance with respect to the main ship. This means that the desired trajectory is defined on the actual position of the main ship plus a predefined distance in the body fixed frame of the main ship:

$$
\eta_{d}=\eta_{m}+R\left(\varphi_{m}\right) L
$$

where the vector $\eta_{m}$ is the actual position and heading of the main ship, the vector $\eta_{d}$ is the actual desired position and heading for the supply ship, the vector $L$ is defined as: $L=\left[\begin{array}{lll}x_{b} & y_{b} & d \varphi\end{array}\right]^{T}$ and $R\left(\varphi_{m}\right)$ is the rotation matrix from the body fixed frame of the main ship to the NED frame.

The vector $\mathrm{L}$ represents the distance between the ships, defined by $x_{b}$ and $y_{b}$, and a difference in the heading is defined by $d \varphi$. The difference in the heading would usually set to zero, but for example in towing operations it can be useful to use different headings. 
However, if the goal is to do underway replenishment it is assumed that the ships must move side by side, which means that $L$ is chosen as:

$$
L=\left[\begin{array}{lll}
0 & y_{b} & 0
\end{array}\right]^{T}
$$

Because only the positions of the ships can be measured an observer scheme is used to estimate the synchronization position error, synchronization velocity error, the position and velocity of the supply ship and the position, velocity and acceleration of the desired trajectory, which are necessary in the feedback control law.

The feedback control law depends on the estimated velocity and acceleration of the desired trajectory, the estimated synchronization velocity error and the measured synchronization position error, respectively $\hat{\dot{\eta}}_{d}, \hat{\vec{\eta}}_{d}, \hat{\dot{e}}$ and $e$. The synchronization errors $e$ and $\hat{\dot{e}}$ are defined as:

$$
e=\eta_{s}-\eta_{d} \quad \hat{\dot{e}}=\hat{\dot{\eta}}_{s}-\hat{\dot{\eta}}_{d}
$$

Furthermore the control law utilizes the dynamic model of the supply ship, depending on the measured position and heading of the supply ship $\eta_{s}$ and the velocity of the supply ship in the body fixed frame $\hat{v}$. The velocity of the supply ship in the body fixed frame is based on the estimated velocity and measured heading of the supply ship in the NED frame:

$$
\hat{v}=R\left(\varphi_{s}\right)^{T} \hat{\dot{\eta}}_{s}
$$

It is assumed that the damping is linear, therefore only the first order terms of the nonlinear damping matrix are used in the damping matrix $D_{s}^{*}\left(\eta_{s}\right)$.

$$
\tau_{s}=M_{s}^{*}\left(\eta_{s}\right) \hat{\ddot{\eta}}_{d}+C_{s}^{*}\left(\hat{v}, \eta_{s}\right) \hat{\dot{\eta}}_{d}+D_{s}^{*}\left(\eta_{s}\right) \hat{\dot{\eta}}_{d}-K_{d} \hat{\dot{e}}-K_{p} e
$$

The gain matrices $K_{p}$ and $K_{d}$ are chosen positive definite and symmetric.

The synchronization position and velocity errors are estimated using a full state nonlinear Luenberger observer.

$$
\begin{aligned}
& \frac{d}{d t} \hat{e}=\hat{\dot{e}}+\Lambda_{1} \widetilde{e} \\
& \frac{d}{d t} \hat{\dot{e}}=-M_{s}^{*}\left(\eta_{s}\right)^{-1}\left[C_{s}^{*}\left(\hat{v}, \eta_{s}\right) \hat{\dot{e}}+D_{s}^{*}\left(\eta_{s}\right) \hat{\dot{e}}+K_{d} \hat{\dot{e}}+K_{p} \hat{e}\right]+\Lambda_{2} \tilde{e}
\end{aligned}
$$

where $\widetilde{e}$ is defined as: $\widetilde{e}=e-\hat{e}$ 
A full state nonlinear observer estimates the position and velocity of the supply ship.

$$
\begin{aligned}
& \frac{d}{d t} \hat{\eta}_{s}=\hat{\dot{\eta}}_{s}+L_{p 1} \widetilde{\eta}_{s} \\
& \frac{d}{d t} \hat{\dot{\eta}}_{s}=-M_{s}^{*}\left(\eta_{s}\right)^{-1}\left[C_{s}^{*}\left(\hat{v}, \eta_{s}\right) \hat{\dot{e}}+D_{s}^{*}\left(\eta_{s}\right) \hat{\dot{e}}+K_{d} \hat{\dot{e}}+K_{p} e\right]+L_{p 2} \widetilde{\eta}_{s}
\end{aligned}
$$

where $\widetilde{\eta}_{s}=\eta_{s}-\hat{\eta}_{s}$

Because the dynamic model of the main ship is unknown, the position, velocity and acceleration should be reconstructed using the estimated states of the supply vessel and the estimated states of the synchronization error. This results in the following estimates:

$$
\begin{aligned}
& \hat{\eta}_{d}=\hat{\eta}_{s}-\hat{e} \\
& \hat{\dot{\eta}}_{d}=\hat{\dot{\eta}}_{s}-\hat{e} \\
& \hat{\ddot{\eta}}_{d}=-\left(M_{s}^{*}\left(\eta_{s}\right)^{-1} K_{p}+\Lambda_{2}\right) \widetilde{e}+\Lambda_{2} \widetilde{\eta}_{s}
\end{aligned}
$$

Rodriguez-Angeles (2002), Nijmeijer and Rodriguez-Angeles (2003) and Kyrkjebø and Pettersen (2003) proved the following. Assume there exist $V_{m}, A_{m}>0$ such that

$$
\begin{aligned}
& \sup _{t}\left\|\dot{\eta}_{d}(t)\right\|=V_{m}<\infty \\
& \sup _{t}\left\|\ddot{\eta}_{d}(t)\right\|=A_{m}<\infty .
\end{aligned}
$$

Assuming that $\Lambda_{1}=L_{p 1}, \Lambda_{2}=L_{p 2}$ and the gain matrices $L_{p 1}, L_{p 2}, K_{p}$ and $K_{d}$ are chosen symmetric and positive definite, the synchronization closed loop error is semi-globally uniformly ultimately bounded when $\left(\hat{\dot{\eta}}_{d}, \hat{\vec{\eta}}_{d}\right) \neq(0,0)$. If the main ship achieves a steady state point in finite time, then $\left(\hat{\dot{\eta}}_{d}(t), \hat{\ddot{\eta}}_{d}(t)\right)=(0,0)$ for $t \in\left(t_{s}, \infty\right)$, after $t_{s} \geq t_{0}$, being the convergence time of the main ship trajectories. Than semi globally exponential convergence of the synchronization closed loop error is achieved after $t \geq t_{s}$ by using this observer-controller scheme.

\subsection{Practical remarks on the proposed observer-controller scheme}

Using this scheme means that the supply ship is controlled to a desired trajectory, which is based on the actual position of the main ship. This means also that there is only communication from the leader to the follower and not vice versa. So the leader will not react on movements of the follower, which can lead to dangerous situations, for example if the supply ship has a black out on the propulsion system or has problems with the rudders or tunnel thruster.

The only restriction of the desired trajectory is that the velocity and acceleration are bounded to satisfy (3.11) and (3.12). Often the velocity and acceleration are bounded by the maximum velocity and maximum acceleration given by the actuators of the mechanical system. This means that these bounds have a physical interpretation.

If there is a distance between the ships required, as during a replenishment operation, the trajectory of the main ship and the supply ship are not necessarily the same. In Figure 3.2 and Figure 3.3 two 
situations are sketched where the trajectory for the main ship and the desired trajectory for the supply ship are not the same.

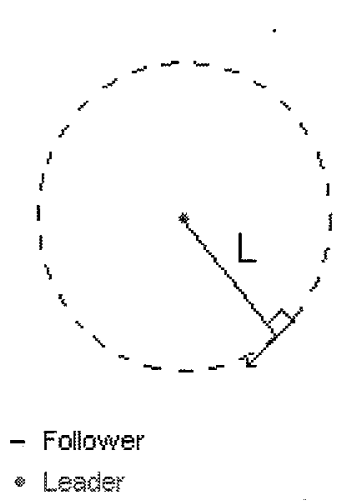

Figure 3.2 Main ship rotates around own centre of gravity.

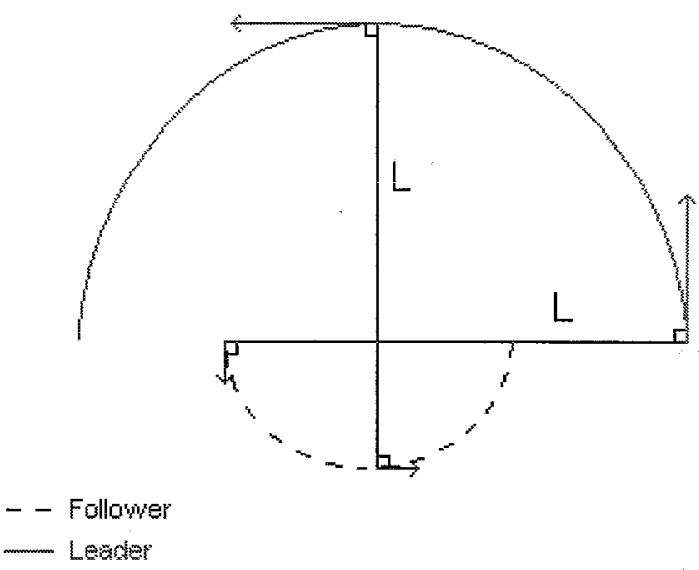

Figure 3.3 Main ship tracks circle with radius smaller than distance between the ships.

In Figure 3.2 the main ship rotates around his own centre of gravity and the supply ship has to follow at a predefined distance $L$ alongside of the main ship. This means that the supply ship has besides a rotation equal to $\dot{\varphi}_{m}$ also a forward velocity of $u_{s}=\dot{\varphi}_{m} L$, while the forward velocity of the main ship is equal to zero. More general, if the main ship trajectory has a non-zero curvature, the supply ship velocity and acceleration will differ from that of the main ship due to the non-zero curvature. If the ships move side by side like by a replenishment operation and track a non-zero curvature, only the forward velocity and forward acceleration of the main ship and supply ship will differ. Care has to be taken to make sure that the desired trajectory for the supply ship is within the physical limitations of this ship otherwise it is impossible to achieve synchronization.

In figure 3.3 the situation is sketched where the distance between the two ships is larger than the radius of the inner bend. If the radius of the inner bend is smaller than the distance between the two ships the supply ship has to move backward to satisfy the synchronization goal. Backward movements should be prevented, because only the dynamic model for forward motion is included.

It is in ship control common to specify and measure the heading between $\pi$ and $-\pi$. This means that there is a discontinuity at $\pi$ and $-\pi$, which can disturb the system. This is a classical problem in ship control and there are no objections to use the standard solution to handle the $\pi /-\pi$ problem in this observer-controller scheme. The standard solution is to define all the errors, control errors as well as observer errors, between $\pi$ and $-\pi$. However in practice after every calculation the heading is specified between $\pi$ and $-\pi$, to make sure that there are no $\pi /-\pi$ problems. If a desired trajectory is defined for the supply ship with a difference in the heading, $\varphi_{d}=\varphi_{m}+d \varphi$, than this desired heading should be specified also between $\pi$ and $-\pi$.

In full scale and during the experiments it is sure that position measurements are not always available. This will disturb the observer scheme and can cause an instable system. In case there are no measurements available the estimated positions, instead of the measured positions, are used in the observer scheme. This means that the observer errors $\widetilde{e}$ and $\widetilde{\eta}_{s}$ become zero and the observations are then only based on the dynamic model of the supply ship. The stability of the observer scheme depends besides the observer gains and the availability of the position measurements on the quality of the dynamical model that is used. 
The proposed observer-controller scheme is a leader-follower scheme. A result of this set up with main ship leader and supply ship follower is that it is not possible to do underway replenishment operations where the supply ship should replenish two main ships. There are than two leaders and one follower, which is not in agreement with this leader-follower synchronization setup. However it is possible to replenish one main ship by two or more supply ships. So, it is important to think about which ship should be the leader.

The advantage of making the supply ship the leader is that with only the dynamic model of the supply ship available, it is possible to replenish all kinds of other ships. So it is possible to replenish different ships with still one supply ship, where only the position of the replenished ship is known and not the dynamic model of this ship.

\subsection{Summary of sufficient conditions}

The goal of this section is to summarize the conditions, which are sufficient to guarantee that the synchronization closed loop error is semi-globally uniformly ultimately bounded during trajectory tracking and semi globally exponential convergence of the synchronization closed loop error is achieved during set point regulation.

If it is possible to satisfy these conditions, this observer-controller scheme can be useful to use.

The dynamic models of the foliowers are available.

The followers are fully actuated systems.

Position measurements of the followers and position information of the desired trajectory must be available.

- $\quad$ The gain matrices $L_{p 1}, L_{p 2}, K_{p}$ and $K_{d}$ are chosen symmetric, positive definite and with respect to the conditions given by the gain tuning procedure of Roderiguez-Angeles (2002) (Nijmeijer and Rodriguez-Angeles, 2003). The gain tuning procedure of Roderiguez-Angeles is given in appendix $B$.

The velocity and acceleration of the desired trajectory are bounded, i.e. the conditions given by (3.11) and (3.12) are satisfied.

- The velocity and acceleration of the desired trajectory are within the physical limitations of the follower. 


\section{Chapter 4}

\section{The MClab: Simulation and experimental setup}

In section 4.1 an overview is given about the equipment, which is used during the experiments. The implementation of the scheme, the task of the main ship and the interpretation of the simulation and experimental results are described in section 4.2 .

\subsection{The MClab: equipment}

The experiments are carried out in the MClab located at NTNU Tyholt, Trondheim. The MClab consist of a $40 \mathrm{~m} \times 6.45 \mathrm{~m}$ basin, a measurement system, a laptop to run the user interface, a wave maker and a model ship. The model ship used during these experiments is Cybership II.

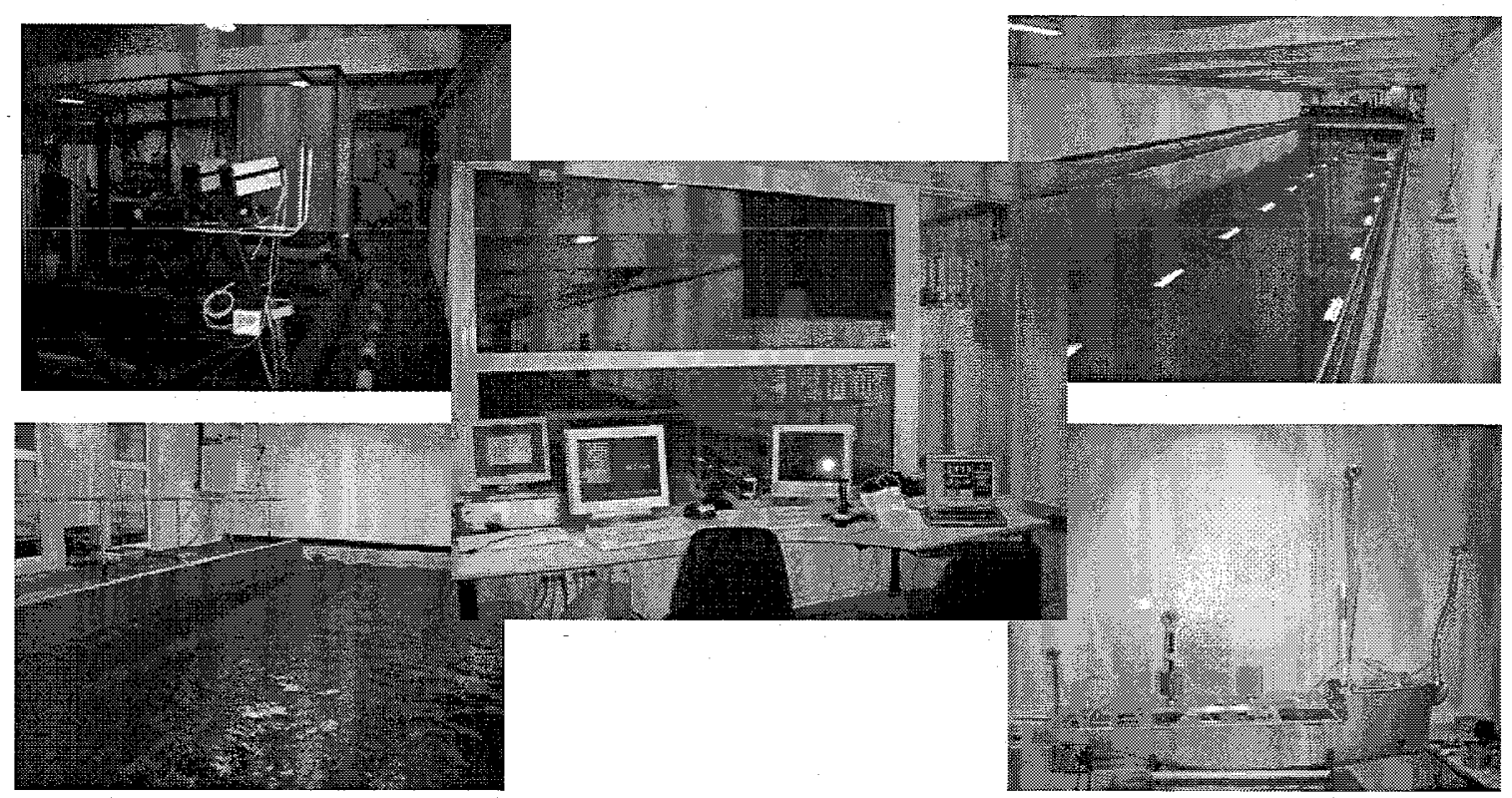

Figure 4.1 System overview of the MCLab.

The position of the ship is measured by a Proreflex motion capture system. This system consists of 4 cameras with a Proreflex Processor Unit, 4 active/passive responders on board Cybership II and a position measurement program NyPOS running on a computer. During the experiments the measurement frequency is $15 \mathrm{~Hz}$. Because only position measurements are available in an area of $5 \mathrm{~m} \times 12 \mathrm{~m}$, only this area is used to carry out the experiments.

A Dell Latitude D800 laptop with a $1.60 \mathrm{GHz}$ Intel Pentium M processor and 512 MB RAM, working under Microsoft Windows XP Professional ver. 2002, is used to control the experiments. With a Labview ver. 6.2 interface it is possible to control the ship by manual inputs, a joystick or an automatic controller. The laptop is also used to build the observer-controller scheme in Matlab ver. 6.5.0 Release 13 and Simulink ver. 5.0. OPAL-RT ver. 6.2 is used to generate make-files and transmits these make-files over a wireless network to the computer onboard Cybership II. Thereafter these files are compiled onboard Cybership II. 
Eventually the system build in Simulink is running onboard Cybership II at $20 \mathrm{~Hz}$, while OPAL-RT is working on the laptop and takes care for the interconnection between Simulink and the Labview interface.

The wireless network is a Breezecom network with a bandwidth of $2 \mathrm{Mbit} / \mathrm{s}$.

The basin is also equipped with a DHI wave maker system. This system can generate predefined, regular or irregular, 2D waves in the basin.

Cybership II is a model supply ship Froude scaled 1:70.

$$
F r=\frac{u}{\sqrt{g L}}
$$

The length of the model ship is $1.3 \mathrm{~m}$ and the weight is about $24 \mathrm{~kg}$. The experiments are carried out at speeds between the $0.1 \mathrm{~m} / \mathrm{s}$ and $0.4 \mathrm{~m} / \mathrm{s}$. In case Cybership II is length scaled it represents a supply vessel of about 87.5 meter. The actual speed in full scale will be than approximately between the 0.84 $\mathrm{m} / \mathrm{s}$ and $3.3 \mathrm{~m} / \mathrm{s}$, respectively. If Cybership II is velocity scaled it represents a supply vessel with a length of more than 6 kilometres, which is not realistic. To verify these experiments in full scale a supply vessel of about 87.5 meter is needed.

Five actuators actuate the ship. At the stern there are two rpm controlled screws with rudders attached. In the bow a two blade rpm controlled tunnel thruster is located. The maximum actuated surge force is 2 $\mathrm{N}$, the maximum sway force is $1.5 \mathrm{~N}$ and the maximum yaw moment is about $1.5 \mathrm{Nm}$. The dynamics of Cybership II can be modelled in the body fixed frame by:

$$
M \dot{v}+C(v) v+D v=\tau_{v}
$$

$M=\left[\begin{array}{ccc}25,8 & 0 & 0 \\ 0 & 33,8 & 1,0115 \\ 0 & 1,0115 & 2,76\end{array}\right]$

$C(v)=\left[\begin{array}{ccc}0 & 0 & -33,8 v-1,0115 r \\ 0 & 0 & 25,8 u \\ 33,8 v+1,0115 r & -25,8 u & 0\end{array}\right]$

$D=D(v)=\left[\begin{array}{ccc}0,72+1,33|u|+5,87 u^{2} & 0 & 0 \\ 0 & 0,86+36,28|v| & -0,11 \\ 0 & -0,11-5,04|v| & 0,5\end{array}\right]$

Because linear damping is assumed only the first order terms of the nonlinear D matrix (4.3) are used. The dynamic model presented here is only valid for forward velocities, which means that backward motion of the supply ship as described in section 3.2 should be prevented. 
There are more actuators than degrees of freedom and the forces of the actuators are not always in the controlled directions, this needs that the calculated control forces have to be distributed to the actuators.

$$
\tau_{v}=T u
$$

$$
\left[\begin{array}{c}
\tau_{x} \\
\tau_{y} \\
\tau_{\varphi}
\end{array}\right]=\left[\begin{array}{ccccc}
1 & 1 & 0 & 0 & 0 \\
0 & 0 & 1 & 1 & 1 \\
L_{y} T_{1} & L_{y} T_{2} & L_{x} T_{3} & L_{x} R_{1} & L_{x} R_{2}
\end{array}\right]\left[\begin{array}{c}
T_{1}\left(\omega_{1}, \delta_{1}\right)-D_{1}\left(\omega_{1}, \delta_{1}\right) \\
T_{2}\left(\omega_{2}, \delta_{2}\right)-D_{2}\left(\omega_{2}, \delta_{2}\right) \\
T_{3}\left(\omega_{3}\right) \\
L_{1}\left(\omega_{1}, \delta_{1}\right) \\
L_{2}\left(\omega_{2}, \delta_{2}\right)
\end{array}\right]
$$

Here $T_{i}$ represents the nominal thrust (rudder angles $\delta_{i}=0$ ) for the left and right screw/rudder pair and the tunnel thruster, $i=1,2,3$ respectively. The additional rudder lift and drag forces are represented by $L_{i}$ and $D_{i}$ for $\mathrm{i}=1,2$. More details about the nominal thrust and lift and drag forces are given in appendix $C$.

To distribute the forces to the different actuators a force allocation algorithm is used. During the simulations and experiments the thrust allocation developed by Karl-Petter Lindegaard, called the KPL thrust allocation, is used. This thrust allocation can be divided into two parts: Force allocation and Inverse mapping. The force allocation distributes the computed control forces to the available actuators. The inverse mapping deals with finding the actuator set points, which will actuate the desired force. The KPL thrust allocation is designed for a ship with rudders at low speeds and tries to minimize the energy consumption. (Lindegraad K.-P., 2003)

During the simulations and experiments it is seen that this force allocation does not work correctly in the backward motion. The problem is that the rudders work like there is a forward motion, while if there is a backward motion the rudders should work in the opposite way. This means that the forces actuated by the rudders in the backward motion have the wrong sign and amplify the error. In contradiction to the rudders the thrusters work correct in the backward motion.

On board Cybership II a $300 \mathrm{MHz}$ computer is located which runs the QNX 6.2 real-time operating system. This computer runs the observer-controller scheme and communicates with the steppermotors of the rudders and the servomotors controlling the rpms of the screws and tunnel thruster through an $\mathrm{H}$ bridge circuit.

As described above the MCLab is build up about different systems, which have to communicate with each other. If there are problems during the experiments it is often a communication problem or it is a computer problem. Many of these problems are easily solved if known what to do. Therefore in appendix $D$ all errors, occurred during the simulations and experiments, and possible solutions are listed. Appendix E gives the IP address, usernames and passwords of the different computers.

\subsection{Simulation and experimental setup}

In Figure 4.1 the Labview screen is shown during a simulation. The trajectory for the main ship is defined by waypoints, where a $7^{\text {th }}$ order polynomial is fitted through. For additional information about the Labview interface and the guidance system the reader is referred to Corneliussen (2003). 


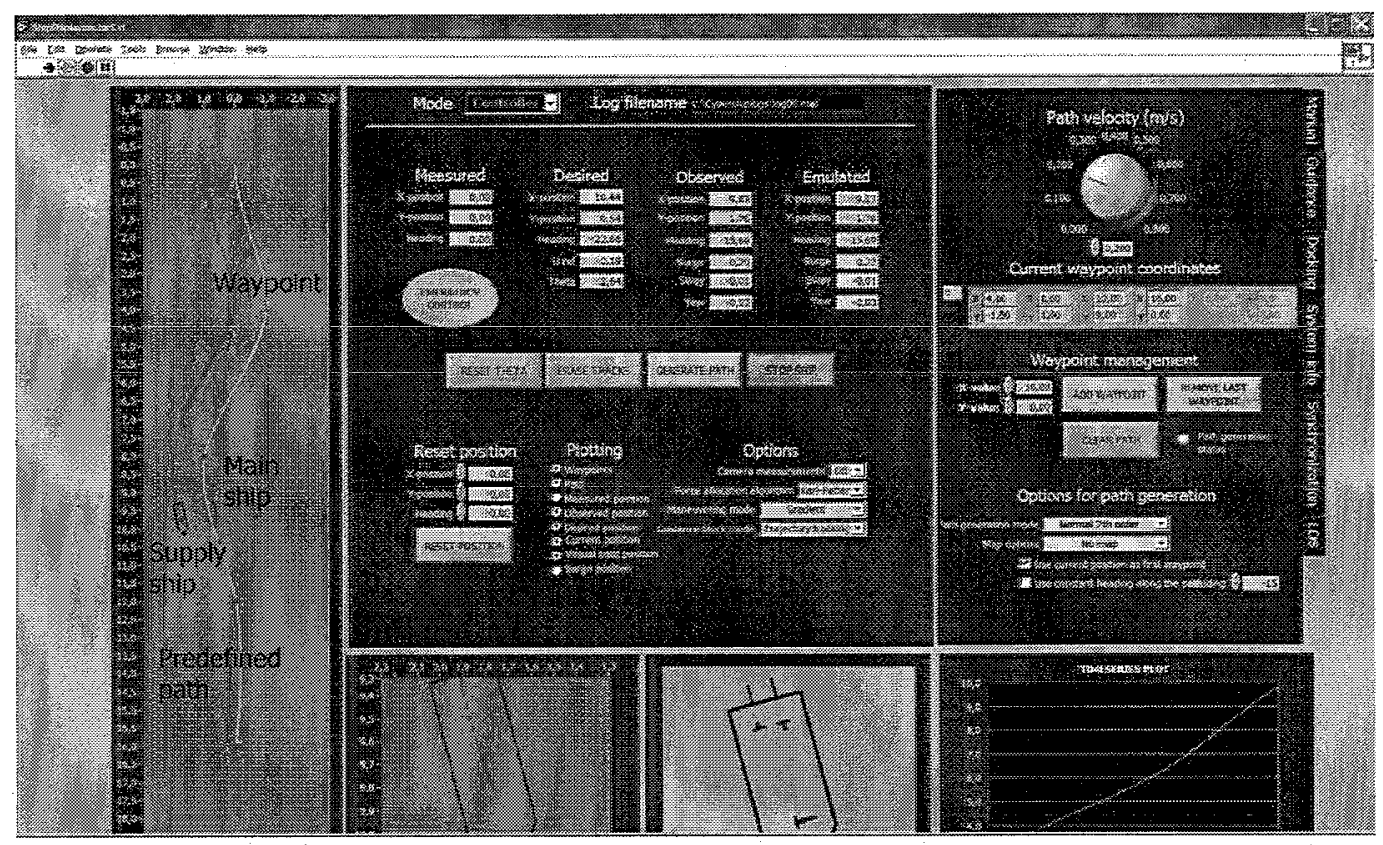

Figure 4.2 Labview interface during a simulation.

Because only one model ship is available at the MClab a virtual ship on the laptop is used to simulate the main ship, while Cybership II is used to represent the supply vessel. The virtual main ship is based on the theoretical ship model of Cybership II ((4.2) and (4.3)) and tracks a predefined path using a backstepping controller (Skjetne et al. 2003a) (Skjetne et al. 2003b).

The position "measurement" of the virtual main ship is used in the observer-controller scheme to define the desired trajectory for the supply ship. "These "measurements" are given without disturbances to the observer controller scheme. In Figure 4.1 the main ship tracks the predefined path, while the supply ship follows at 1 meter alongside the main ship.

Because the main ship is a virtual ship during the experiments, there are no physical interactions between the main ship and supply ship, like for example Venturi effects and forces actuated by the cables and hoses of the replenishment rig. This means that the experiments are not representative for a real underway replenishment operation, but should be interpreted as regular trajectory tracking and position keeping. Of course there is synchronization, because the desired trajectory for the supply ship is based on the actual position of the virtual main ship. The synchronization is created by the definition of the desired trajectory for the supply ship and has nothing to do with the observer-controller scheme itself. If an arbitrarily trajectory is defined with only positions and the conditions given in section 3.3 are satisfied the observer-controller scheme should be able to follow this path.

During the experiments with waves only the supply ship experiences these waves, because the main ship moves in a virtual calm sea. The waves can be interpreted as disturbances on the observer-controller scheme, which means that these experiments give some information about the robustness of the scheme. 


\section{Chapter 5}

\section{Underway replenishment: position keeping}

In this chapter some background will be given about the tuning of the proposed observer-controller scheme and the performance during position keeping. At the moment there is no systematic gain tuning procedure available, this means that the tuning of the scheme is an arduous and time-consuming task. In section 5.1 it is explained why it is necessary to choose the control gains in the body fixed frame, while in section 5.2 the influence of the different observer and controller gains on the performance of the observer-controller scheme is discussed. In section 5.3 the results obtained with the gain set for trajectory tracking as well as position keeping are presented, while in section 5.4 the results obtained with the gain set developed especially for position keeping are presented. To verify the exponential convergence during position keeping, the experimental results are compared with simulation results under ideal conditions. Furthermore the problems with the gain set for trajectory tracking as well as position keeping are explained and how these problems are caused and solved. In section 5.5 the performance of both gain sets during position keeping is assessed.

\subsection{Behaviour of ships}

Before tuning the observer-controller scheme some remarks about the behaviour of a ship are made. Because some directions of a ship are directly actuated $\left(\mathrm{x}_{\mathrm{b}}\right.$ and $\varphi$ ), whereas other directions are indirectly actuated $\left(y_{b}\right)$, a ship moves in some directions easier than in other directions. Therefore it will be preferable to use a specific sequence of control to reach a desired point (position keeping). If a ship should move to a desired point in the $y_{b}$-direction, it will be easy to have some forward speed. If the desired $y_{b}$ point is reached it will be preferable to sail in a straight line to the desired $x_{b}$ point. This is only possible if the $\varphi$ direction can be chosen arbitrarily. If the ship has arrived the desired point, it can use its tunnel thruster to reach its desired orientation $\varphi$. So the preferred specific sequence of control to reach a desired point would be $y_{b}, x_{b}$ and finally the $\varphi$ orientation. During trajectory tracking this specific sequence of control is not so clear. If the ship tracks the path with an error in the heading, there occurs an error in the sideward direction $y_{b}$ as well. This means that during trajectory tracking the control of the heading is more important than during position keeping.
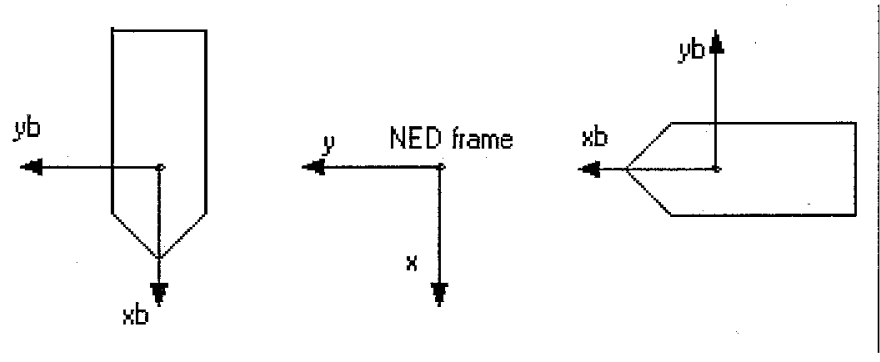

Figure 5.1a and 5.1b Different orientations in the NED frame

The controller is specified as:

$$
\begin{aligned}
& \tau_{s}=M_{s}\left(\eta_{s}\right) \hat{\ddot{\eta}}_{d}+C_{s}\left(\eta_{s}, \hat{\dot{\eta}}_{s}\right) \hat{\dot{\eta}}_{d}+D_{s}\left(\eta_{s}, \hat{\dot{\eta}}_{s}\right) \hat{\dot{\eta}}_{d}-K_{d} \hat{\dot{e}}-K_{p} e \\
& \eta=\left[\begin{array}{lll}
x & y & \varphi
\end{array}\right]^{T}
\end{aligned}
$$


This implies that the gains $K_{d}$ and $K_{p}$ are constant and independent of the position of the ship, while the dynamic model is dependent on the position of the ship (2.14). It is very difficult to tune the observercontroller scheme using constant control gains in the NED frame. The best results are obtained by using the same control gains for the $x$ - and $y$-directions. The best results represent here more something like the least bad results. This can be explained by the dynamics of the ship in the NED coordinates.

If a ship moves in the position as sketched in Figure 5.1a then the differential equation in the $x$-direction of the dynamic model in the NED frame represents the forward motion of the ship. This means that the control law in the $x$-direction controls the forward motion of the ship. On the other hand in the $y$-direction the dynamic model in the NED frame represents the sideward motion of the ship, so the control law in ydirection controls the sideward motion of the ship. If the position of the ship changes to the position sketched in Figure 5.1b, than the dynamic model in the NED frame describes in the $x$-direction the sideward motion of the ship and in the $y$-direction the forward motion of the ship. This means that the control law in the $x$-direction now controls the sideward motion of the ship and the control law in the $y$ direction now controls the forward motion of the ship, while the gains in the $x$ - as well as the $y$-direction are independent of the position of the ship (5.1).

If the gains are chosen independent of the position of the ship as in (5.1), then the gain in the $x$-direction as well as the gain in the $y$-direction should be able to control all different motions. Therefore the gains in the $x$ - and $y$-direction have to be the same. It may be clear that this is not optimal.

Because the dynamics in the body fixed frame are independent of the position of the ship, it may be an option to specify the control gains in the body fixed frame and transiate them to the NED frame. It is then possible to specify gains for the forward motion, the sideward motion as well as for the heading of the ship. In addition it is now possible to give a physical interpretation on the gains, which makes the tuning procedure more understandable. To transform the control gains with respect to the body fixed frame to control gains in the NED frame, the rotation matrix $R\left(\varphi_{s}\right)$ is used.

In the observer-controller scheme $((3.5),(3.6),(3.8)$ and $(3.10))$ the $K_{p}$ gain as well as the $K_{d}$ gain has to be replaced by:

$$
\begin{aligned}
& K_{p}=R(\varphi) K_{p, b} R(\varphi)^{T} \\
& K_{d}=R(\varphi) K_{d, b} R(\varphi)^{T}
\end{aligned}
$$

where $K_{p, b}$ and $K_{d, b}$ are respectively the proportional control gain and the differential control gain in the body fixed frame.

For the observer gains it is not so evident to choose the gains in the body fixed frame or the NED frame. The observer gains will be chosen directly with respect to the control gains to make sure that the observer is faster than the controller. This means that the observer gains should be chosen in the body fixed frame, because then the observer gains depend on the position of the ship in the same way as the controller gains in (5.3) and (5.4). However, this means that the estimated values in the $x$-direction of the NED frame also depend on the estimation errors in the $y$-direction of the NED frame, which is not desirable.

This means that there is a contradiction: The observer gains chosen in the body fixed frame are chosen directly with respect to the control gains, but the estimated values depend also on the estimation errors in the other directions. Or the observer gains chosen in the NED frame, which are chosen not directly with respect to the control gains, but the estimated values do not depend on the observer errors in the other directions.

In case of very different control gains in the forward and sideward direction of the body fixed frame, it might be useful to choose the observer gains in the body fixed frame and transform them to the NED frame. The observer gains depend now on the position of the ship in the same way as the controller gains 
((5.3) and (5.4)), which means that the observer gains can be chosen directly with respect to the controller gains. However, during the tuning of the scheme it is seen that choosing the observer gains in the NED frame is not a problem. Therefore the observer gains are chosen in the NED frame.

\subsection{Influence of observer and controller gains on the performance of the scheme}

The scheme is tuned during simulations, where nonlinear damping is assumed for the simulated Cybership II. During the simulations it is seen that for practical applications it is useless to tune without measurement noise in the system. It is known that the standard deviation of the position measurements in the $x$-direction is about $0.81 \mathrm{~mm}$ (Sveen, 2003). This makes it possibie to model the measurement noise as white noise with a noise power equals to:

$P_{\omega n}=s t d^{2} t_{c}$

where $t_{c}$ is the correlation time, which is equal to the sampling time. Because simulink is working at 20 $\mathrm{Hz}$, the noise power during the simulations with measurement noise on the position measurements of the supply ship is set as $3.280510^{-8}$. It is seen that the measurement noise causes much larger and highly fluctuating control actions than is expected on the basis of the simulations without measurement noise.

In Rodriguez-Angeles (2002) and Nijmeijer and Rodriguez-Angeles (2003) conditions for the observer gains and control gains are given to ensure stability and boundedness of the synchronization system (appendix B). Because this theorem proves only stability and boundedness of the synchronization system and says nothing about the size of the bound, the tuning of the observer controller scheme is trial and error based.

One of the difficulties during the tuning procedure is how to choose $L_{p 1}$. If the $L_{p 1}$ gain is chosen too low, it causes slightly damped oscillations in the system. In addition increasing $L_{p 1}$ will result in a larger position error bound. This means that the $L_{p 1}$ gain should be chosen as low as possible, but not so low that the slightly damped oscillations in the system occur.

There is also a difficulty in the $L_{p 2}$ gain. Higher $L_{p 2}$ gain will result in better estimates of the velocity. Increasing accuracy of the estimated velocities will result in better estimated positions and lower position error bounds. However increasing the $L_{p 2}$ gain will also introduce measurement noise to the observed velocity, which can lead to highly fluctuating control actions.

The error bound can be made smaller by increasing the $K_{p}$ gain. The $K_{d}$ gain is chosen to ensure that there is enough damping in the system. Increasing the $K_{d}$ gain will in addition increase the region of attraction, but will also amplify the measurement noise in the system. In appendix $\mathrm{F}$ a table is presented where the influence of changing the different observer and controller gains can be seen.

A rule of thumb in control engineering is that the observer is faster than the controller. Since the system is nonlinear it is not possible to verify this directly. Therefore the system is linearized around its defined set point, which in this case is the origin: $\eta=\left[\begin{array}{ccc}0 & 0 & 0\end{array}\right]^{T}, \dot{\eta}=\left[\begin{array}{lll}0 & 0 & 0\end{array}\right]^{T}$. This linearized system is used to calculate the controller and observer poles (appendix $G$ ) to verify if the observer is faster than the controller.

There are several sets of gains possible. The gain sets used are chosen by trial and error. Since the system is nonlinear and coupled, the only way to improve the performance of the system is by online tuning, i.e. tuning while the system is running. This makes the gain tuning procedure an arduous task. In Manssouri (2002) a systematic way to improve the performance of the system is described. This systematic way is based on tuning the linearized system and after that improving the system by online tuning. This systematic way can be seen as the first step in the way to get a systematic gain tuning procedure, which satisfies the conditions given by Rodriguez-Angeles (2002) (Nijmeijer and RodriguezAngeles, 2003) and predicts the size of the error bounds. 
After a lot of simulations (appendix $K$ ) the following set of gains is found for position keeping and trajectory tracking:

Set 1:

$$
\begin{array}{ll}
K_{p, b}=\operatorname{diag}\left[\begin{array}{lll}
150 & 35 & 5
\end{array}\right] & K_{d, b}=\operatorname{diag}\left[\begin{array}{lll}
200 & 70 & 10
\end{array}\right] \\
L_{p 1}=\operatorname{diag}\left[\begin{array}{lll}
8 & 8 & 2
\end{array}\right] & L_{p 2}=\operatorname{diag}\left[\begin{array}{lll}
100 & 100 & 5
\end{array}\right]
\end{array}
$$

\begin{tabular}{|c|c|c|c|}
\hline direction & poles controller & poles observer supply ship & poles observer synchronization error \\
\hline $\mathrm{x}$ & -0.8375 & $-4.0000+9.1652 \mathrm{i}$ & $-7.8899+10.2860 \mathrm{i}$ \\
& -6.9424 & $-4.0000-9.1652 \mathrm{i}$ & $-7.8899-10.2860 \mathrm{i}$ \\
\hline $\mathrm{y}$ & -0.8174 & $-4.0000+9.1652 \mathrm{i}$ & $-5.0633+9.6080 \mathrm{i}$ \\
& -1.2492 & $-4.0000-9.1652 \mathrm{i}$ & $-5.0633-9.6080 \mathrm{i}$ \\
\hline$\varphi$ & -0.5550 & $-1.0000+2.0000 \mathrm{i}$ & $-2.9210+2.4465 \mathrm{i}$ \\
& -3.3471 & $-1.0000-2.0000 \mathrm{i}$ & $-2.9210-2.4465 \mathrm{i}$ \\
\hline
\end{tabular}

Table 5.1 Poles observer and controller gain set 1 .

In Table 5.1 it is seen that the observer gains are not always faster than the controller gains. It might be better to tune first the linear system to satisfy that the observer gains are faster than the controller gains, then use these gains as starting point and improve the performance of the nonlinear system by online tuning.

\subsection{Experimental results during position keeping: gain set 1}

To verify the theoretical result of global exponential convergence during dynamical positioning the main ship is held at a constant position and heading, while the supply ship is synchronized to a position alongside given by $\eta_{d}=[0,0,0]^{T}$. The main ship is in position $\eta_{m}=[0,-1,0]^{T}$. This means that the supply ship is controlled to a position 1 meter alongside the starboard side of the main ship. The supply ship starts in a position around $\eta_{0}=\left[-1,-1, \frac{1}{2} \pi\right]^{T}$, with initial velocities $\dot{\eta}_{0}=[0,0,0]^{T}$.

Only the position of the main ship can be measured, which implies that it is difficult to verify the velocity synchronization directly. Therefore a back-to-back comparison with simulations under ideal conditions is done. Ideal conditions mean that the model used in the observer-controller scheme is the same as the model used to simulate Cybership II. Cybership II is consequently simulated with only first order damping. Effects of environmental disturbances like wind, waves and current are neglected. While the position "measurements" of the main ship and the supply ship are given without simulated measurement noise to the observer-controller scheme.

The experimentally obtained $e, \widetilde{e}, \widetilde{\eta}_{d}$ and $\widetilde{\dot{\eta}}_{d}$, that are available from the experimental data, are compared to the results of the simulation under ideal conditions. If the results of $e, \widetilde{e}, \widetilde{\eta}_{d}$ and $\widetilde{\dot{\eta}}_{d}$ are similar, it is plausible to think that $\dot{e}$ and $\tilde{e}$ during the experiment are similar to the simulation results under ideal conditions as well. The presented observer errors $\tilde{e}, \tilde{e}, \widetilde{\eta}_{d}$ and $\tilde{\eta}_{d}$ and the control errors $e$ and $\dot{e}$ are calculated in the NED frame. The experimentally obtained $e, \widetilde{e}, \widetilde{\eta}_{d}$ and $\widetilde{\dot{\eta}}_{d}$ are presented in Figure 5.2. The simulation results of the observer errors are presented in Figure 5.3, while Figure 5.4 shows the control errors during the simulation under ideal conditions. 

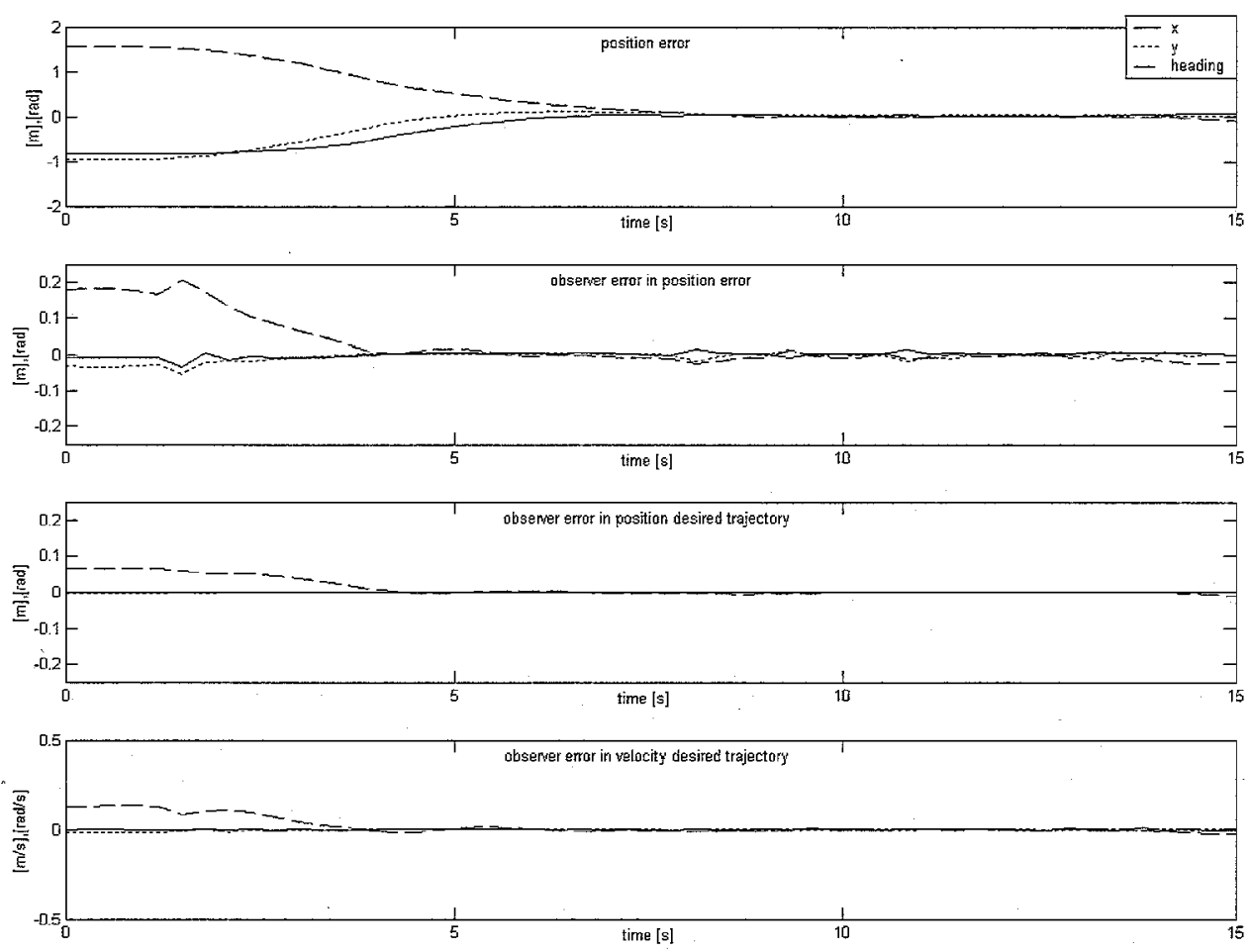

Figure 5.2 Experimental obtained $e, \widetilde{e}, \widetilde{\eta}_{d}$ and $\widetilde{\dot{\eta}}_{d}$ during position keeping: gain set 1 .
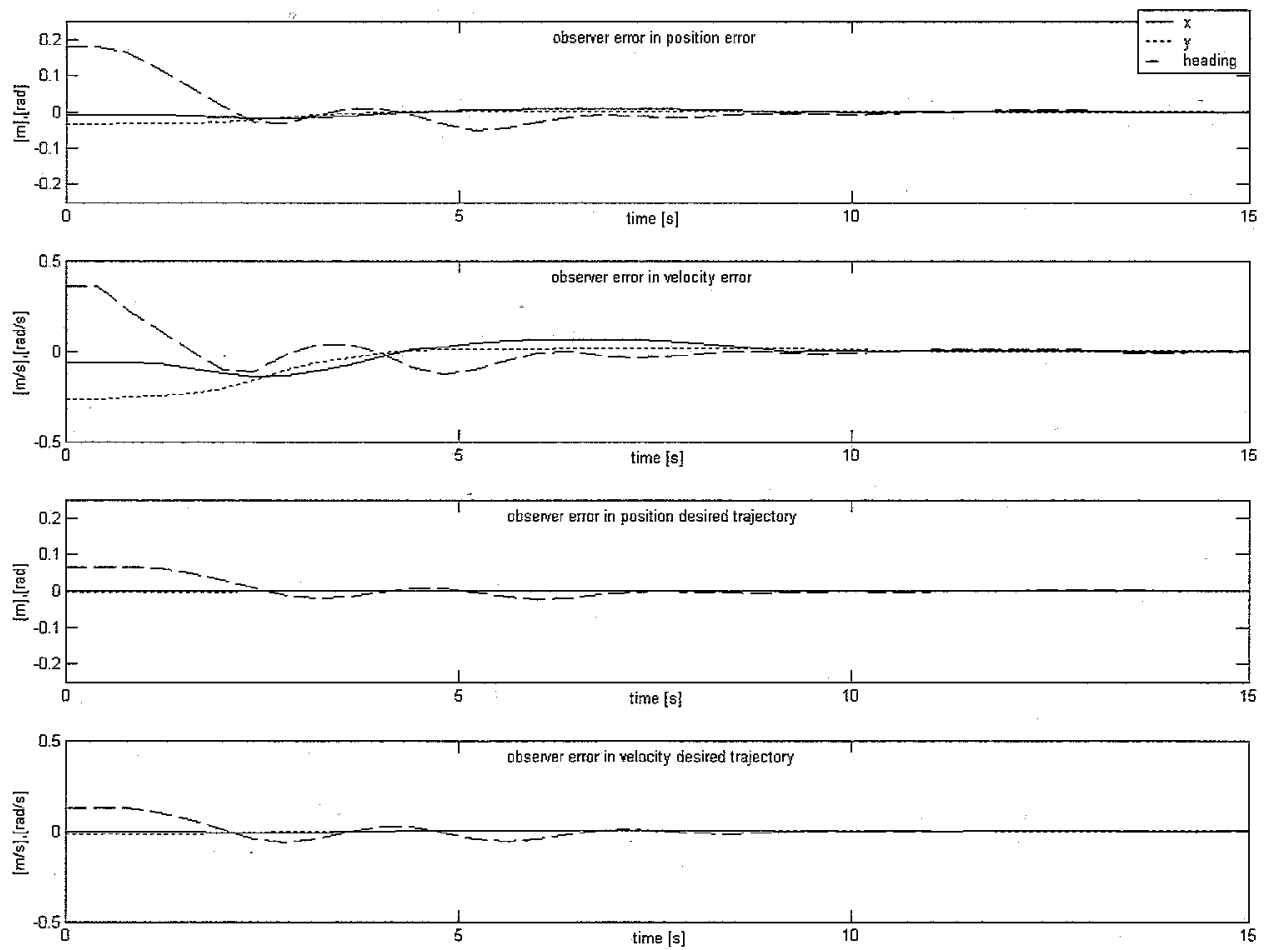

Figure 5.3 Simulation results of $\widetilde{e}, \widetilde{e}, \widetilde{\eta}_{d}$ and $\tilde{\dot{\eta}}_{d}$ during position keeping: gain set 1 . 

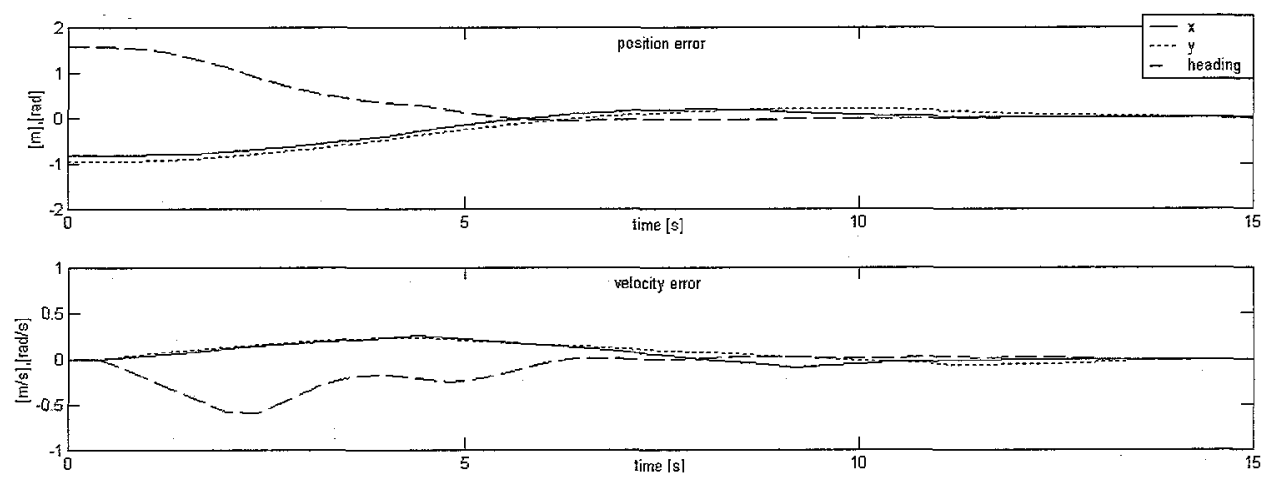

Figure 5.4 Simulation results of $e$ and $\dot{e}$ during position keeping: gain set 1 .

To make the comparison between the experiment and the simulation under ideal conditions quantitative an index is used. The index used in this report is the IAE value (5.7) (Dorf and Bishop, 1998).

$$
L A E=\int_{t_{o}}^{t_{e}}|e| d t
$$

In Table 5.1 the IAE values are given for the closed loop errors $e, \widetilde{e}, \widetilde{\eta}_{d}$ and $\widetilde{\eta}_{d}$ during the experiment and the simulation under ideal conditions. The IAE values are calculated during the first 15 seconds of the experiment and simulation. This means that the calculated IAE values correspond with the total integrated errors in Figure 5.2, Figure 5.3 and Figure 5.4.

\begin{tabular}{|c|l|l|l|l|l|l|l|l|l|l|l|l|}
\hline & IAE $e_{x}$ & IAE $e_{y}$ & IAE $e_{\varphi}$ & IAE $\widetilde{e}_{x}$ & IAE $\tilde{e}_{y}$ & IAE $\widetilde{e}_{\varphi}$ & IAE $\tilde{\eta}_{d x}$ & IAE $\widetilde{\eta}_{d y}$ & IAE $\widetilde{\eta}_{d \varphi}$ & IAE $\tilde{\dot{\eta}}_{d x}$ & IAE $\tilde{\dot{\eta}}_{d y}$ & IAE $\tilde{\dot{\eta}}_{d \varphi}$ \\
\hline experiment & 4.1299 & 3.1920 & 6.9276 & 0.0732 & 0.1389 & 0.5962 & 0.0034 & 0.0061 & 0.2218 & 0.0314 & 0.0475 & 0.4165 \\
simulation & 4.8286 & 3.2540 & 4.6463 & 0.0730 & 0.0990 & 0.4026 & 0.0037 & 0.0055 & 0.1938 & 0.0299 & 0.0418 & 0.3775 \\
\hline
\end{tabular}

Table 5.2 IAE values for the closed loop synchronization errors: $e, \widetilde{e}, \widetilde{\eta}_{d}$ and $\widetilde{\dot{\eta}}_{d}$

It seems that the experimental results agree well with the simulation results. There is not much overshoot during the transient response (Figure 5.2 and Figure 5.4). This is desirable, because of the distance between the two ships is limited and collisions should be avoided. Besides that the lower overshoot is an indication for stability margins with this set of gains for the observer-controller scheme. The overshoot during the simulation under ideal condition is more, because the ship is modelled with only first order damping.

The steady state errors in the experiment do not go to exactly zero, but oscillate around zero. This is caused by the measurement noise, which disturbs the observer-controller scheme. During the simulation under ideal conditions the observer and controller errors go to exactly zero (Table 5.5a and appendix $\mathrm{H}$ ), which agrees with exponential convergence of the synchronization closed loop errors. The experimental results seem to agree with the simulation results under ideal conditions. Therefore the experimental results seem to agree with the theoretic result of exponential convergence as well.

The disadvantage of using gain set 1 , high $L_{p 2}$ gain and high $K_{d}$ gain, is the high control action during position keeping, which can be seen in Figure 5.5. Here the problem of saturated forces is clearly seen. 

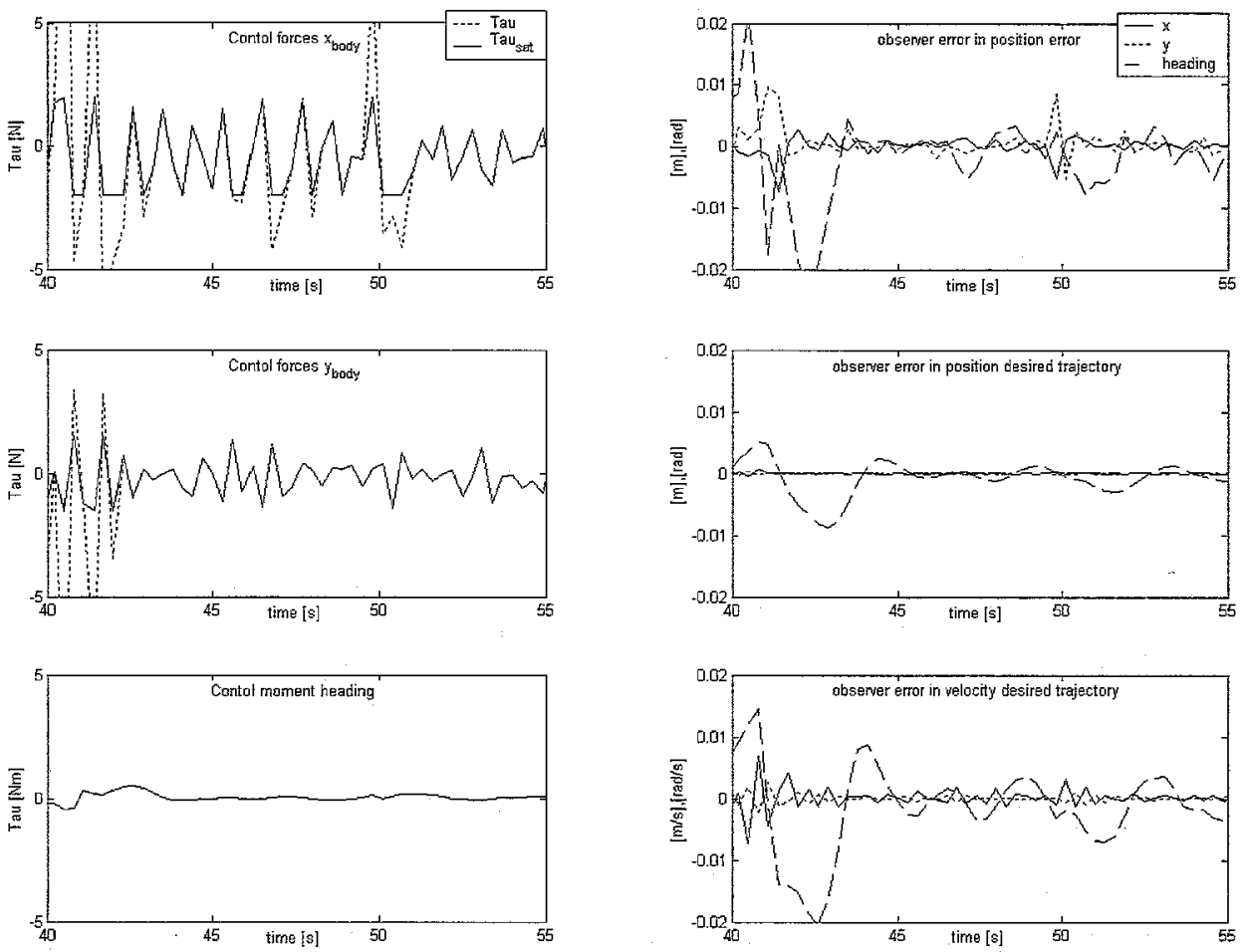

Figure 5.5 Control forces and observer errors $\widetilde{e}, \widetilde{\eta}_{d}$ and $\widetilde{\dot{\eta}}_{d}$ during steady state response: gain set 1 .

The control forces are saturated, which means that the controller has not anymore the full control over the system. Thereafter saturated forces can disturb the observer because in the observer the not saturated control forces are used to calculate the observed states. This means that if the calculated forces are larger than the saturated forces an error is introduced in the observer. This can be seen in Figure 5.5. The large errors in the observer arise at the same time that the control forces are saturated. Besides an error in the observer, larger control errors $e$ and $\dot{e}$ arise as well.

Boundedness of the controls is an important open problem in control theory, since it can generate instability in the closed loop system (Rodrigues-Angeles, 2002) (Nijmeijer and Rodriguez-Angeles, 2003). This problem is beyond the scope of this report, but clearly care has to be taken with saturated and bounded control forces.

\subsection{Experimental results during position keeping: gain set 2}

To reduce the high control forces a set of gains is developed for only position keeping. Important during position keeping is that there is enough damping in the system to get the overshoot in an acceptable range. However not too much damping is desired, because the settling time should be in an acceptable range as well. Because during position keeping there is exponential convergence of the closed- loop errors, the gain $K_{p}$ and $L_{p 2}$ can be chosen lower. This in contradiction with boundedness of the closed-loop errors, where the gains $K_{p}$ and $L_{p 2}$ should be chosen high to make the bound smaller. After a lot of simulations (appendix $\mathrm{K}$ ) the following set of gains is found especially for position keeping:

Set 2:

$K_{p, b}=\operatorname{diag}\left[\begin{array}{lll}35 & 15 & 5\end{array}\right] \quad K_{d, b}=\operatorname{diag}\left[\begin{array}{lll}70 & 40 & 10\end{array}\right]$ 
$\mathrm{L}_{\mathrm{p} 1}=\operatorname{diag}\left[\begin{array}{lll}8 & 8 & 2\end{array}\right] \quad \mathrm{L}_{\mathrm{p} 2}=\operatorname{diag}\left[\begin{array}{lll}10 & 10 & 10\end{array}\right]$

\begin{tabular}{|c|c|c|c|}
\hline direction & poles controller & poles observer supply ship & poles observer synchronization error \\
\hline$x$ & -0.6482 & -1.5505 & $-5.3705+2.1077 \mathrm{i}$ \\
& -2.0929 & -6.4495 & $-5.3705-2.1077 \mathrm{i}$ \\
\hline $\mathrm{y}$ & $-0.6023+0.2863 \mathrm{i}$ & -1.5505 & -3.6096 \\
& $-0.6023-0.2863 \mathrm{i}$ & -6.4495 & -5.6139 \\
\hline$\varphi$ & -0.5514 & $-1.0000+3.0000 \mathrm{i}$ & $-2.9238+3.3005 \mathrm{i}$ \\
& -3.3153 & $-1.0000-3.0000 \mathrm{i}$ & $-2.9238-3.3005 \mathrm{i}$ \\
\hline
\end{tabular}

Table 5.3 Poles observer and controller gain set 2 .

In the $x$-direction and $y$-direction the observer gains are faster than the controller gains of the linearized system. In the $\varphi$ direction, the rule of thumb, faster observer gains than controller gains, is not satisfied with this gain set.

The same experiment as described in section 5.3 is carried out, to verify the theoretical result and assess the performance and control actions during position keeping with this set of gains.

The presented observer errors $\widetilde{e}, \widetilde{\dot{e}}, \widetilde{\eta}_{d}$ and $\widetilde{\dot{\eta}}_{d}$ and the control errors $e$ and $\dot{e}$ are calculated in the NED frame. The figures are scaled similar as in section 5.3. The experimental results of $e, \widetilde{e}, \widetilde{\eta}_{d}$ and $\widetilde{\eta}_{d}$ are presented in Figure 5.6. The simulation results of the observer errors are presented in Figure 5.7, while Figure 5.8 shows the control errors during the simulation under ideal conditions.
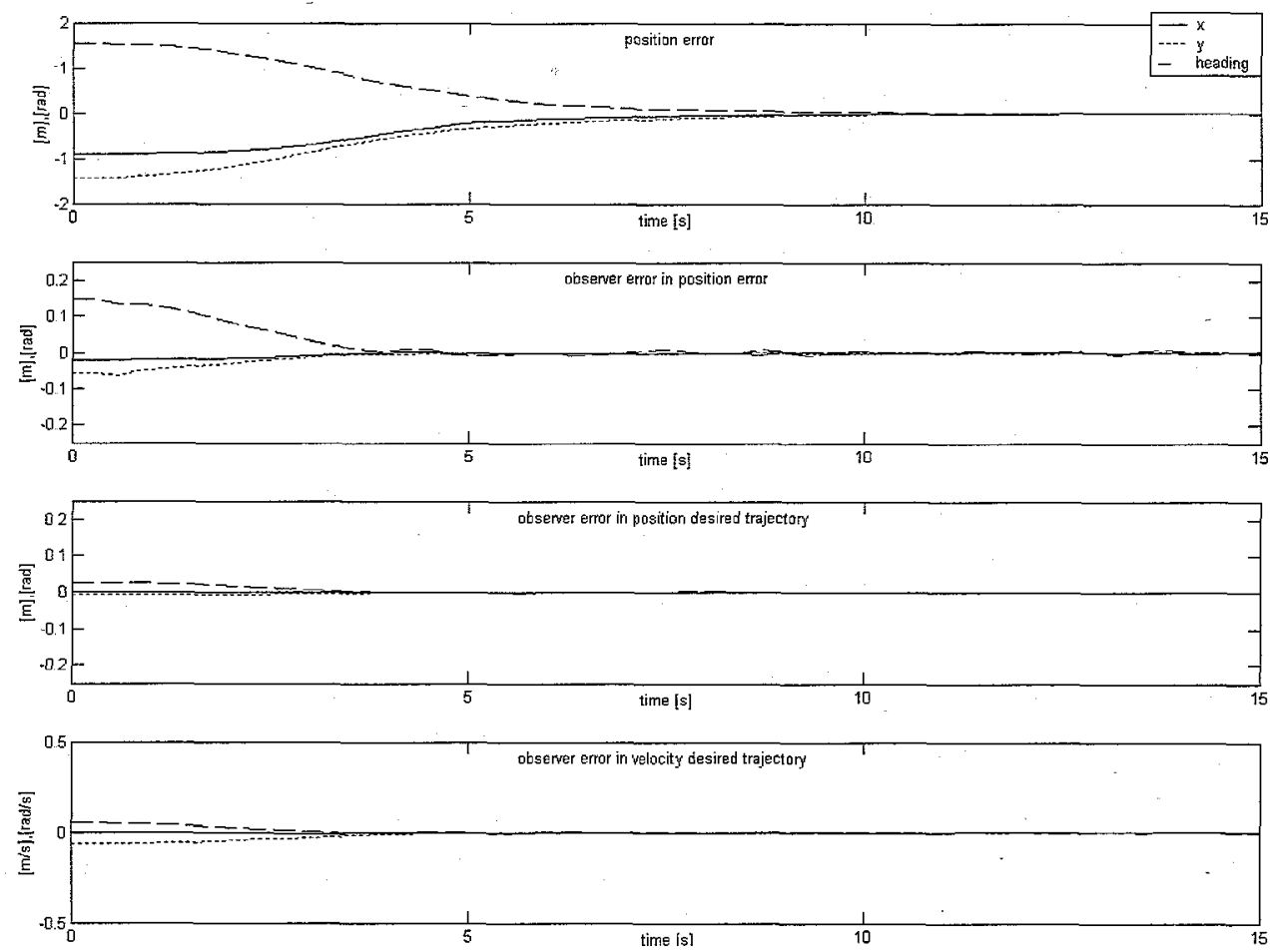

Figure 5.6 Experimental obtained $e, \widetilde{e}, \widetilde{\eta}_{d}$ and $\widetilde{\dot{\eta}}_{d}$ during position keeping: gain set 2 . 

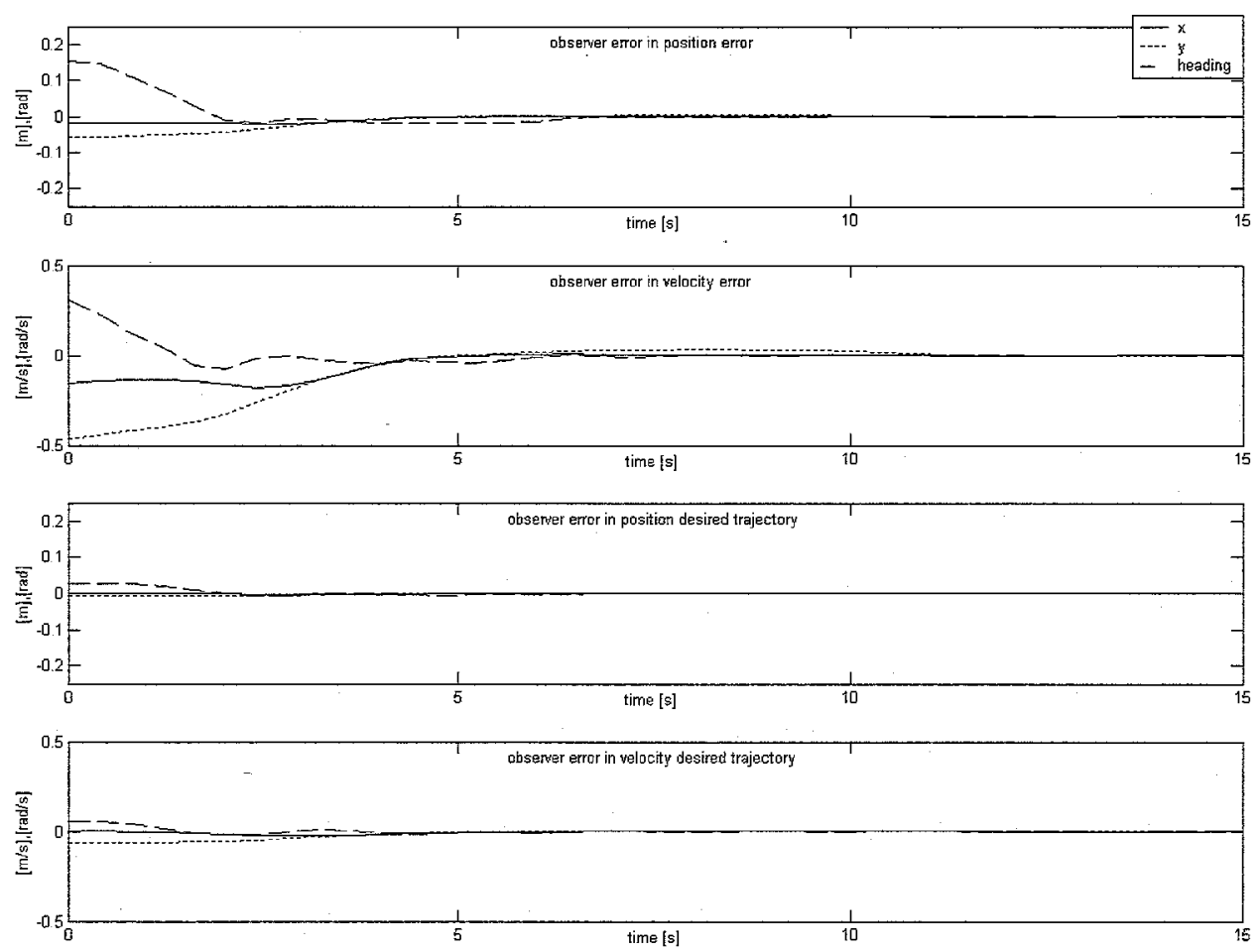

Figure 5.7 Simulation results of $\widetilde{e}, \widetilde{\dot{e}}, \widetilde{\eta}_{d}$ and $\widetilde{\dot{\eta}}_{d}$ during position keeping: gain set 2 .
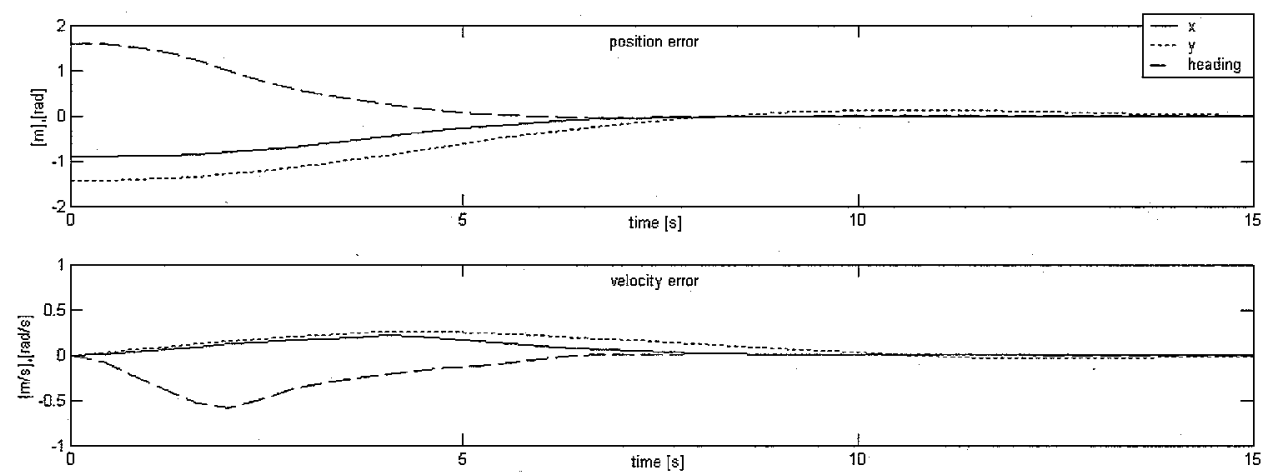

Figure 5.8 Simulation results of $e$ and $\dot{e}$ during position keeping: gain set 2 .

\begin{tabular}{|l|c|c|c|c|c|c|c|c|c|c|c|c|}
\hline & IAE $e_{x}$ & IAE $e_{y}$ & IAE $e_{\varphi}$ & IAE $\widetilde{e}_{x}$ & IAE $\widetilde{e}_{y}$ & IAE $\widetilde{e}_{\varphi}$ & IAE $\tilde{\eta}_{d x}$ & IAE $\widetilde{\eta}_{d y}$ & IAE $\widetilde{\eta}_{d \varphi}$ & IAE $\tilde{\dot{\eta}}_{d x}$ & IAE $\tilde{\dot{\eta}}_{d y}$ & IAE $\tilde{\dot{\eta}}_{d \varphi}$ \\
\hline experiment & 5.9885 & 2.8533 & 6.2076 & 0.0642 & 0.1332 & 0.3602 & 0.0037 & 0.0235 & 0.0718 & 0.0296 & 0.1804 & 0.1360 \\
simulation & 6.0477 & 4.4320 & 4.2070 & 0.0760 & 0.1723 & 0.2491 & 0.0084 & 0.0262 & 0.0551 & 0.0674 & 0.2015 & 0.1065 \\
\hline
\end{tabular}

Table 5.4 IAE values for the closed loop synchronization errors: $e, \widetilde{e}, \widetilde{\eta}_{d}$ and $\widetilde{\dot{\eta}}_{d}$ 
In Table 5.4 the IAE values are given for the closed loop errors $e, \widetilde{e}, \widetilde{\eta}_{d}$ and $\widetilde{\eta}_{d}$ during the experiment and the simulation under ideal conditions. If the experimental results and the simulation results under ideal conditions are compared, it seems that they agree well. The effect of the unmodelled nonlinear damping in the simulated Cybership II during the simulation under ideal conditions is less visible, which indicates that the damping causes by the differential gain dominates the damping in the system. The position error seems to go to zero (Table 5.5b) (appendix $\mathrm{H}$ ) and in contradiction with gain set 1 there is much less oscillation in the position error around zero. It seems that the $L_{p 2}$ gain in the heading is chosen a little too high, which causes the oscillation in the observed states of the heading.
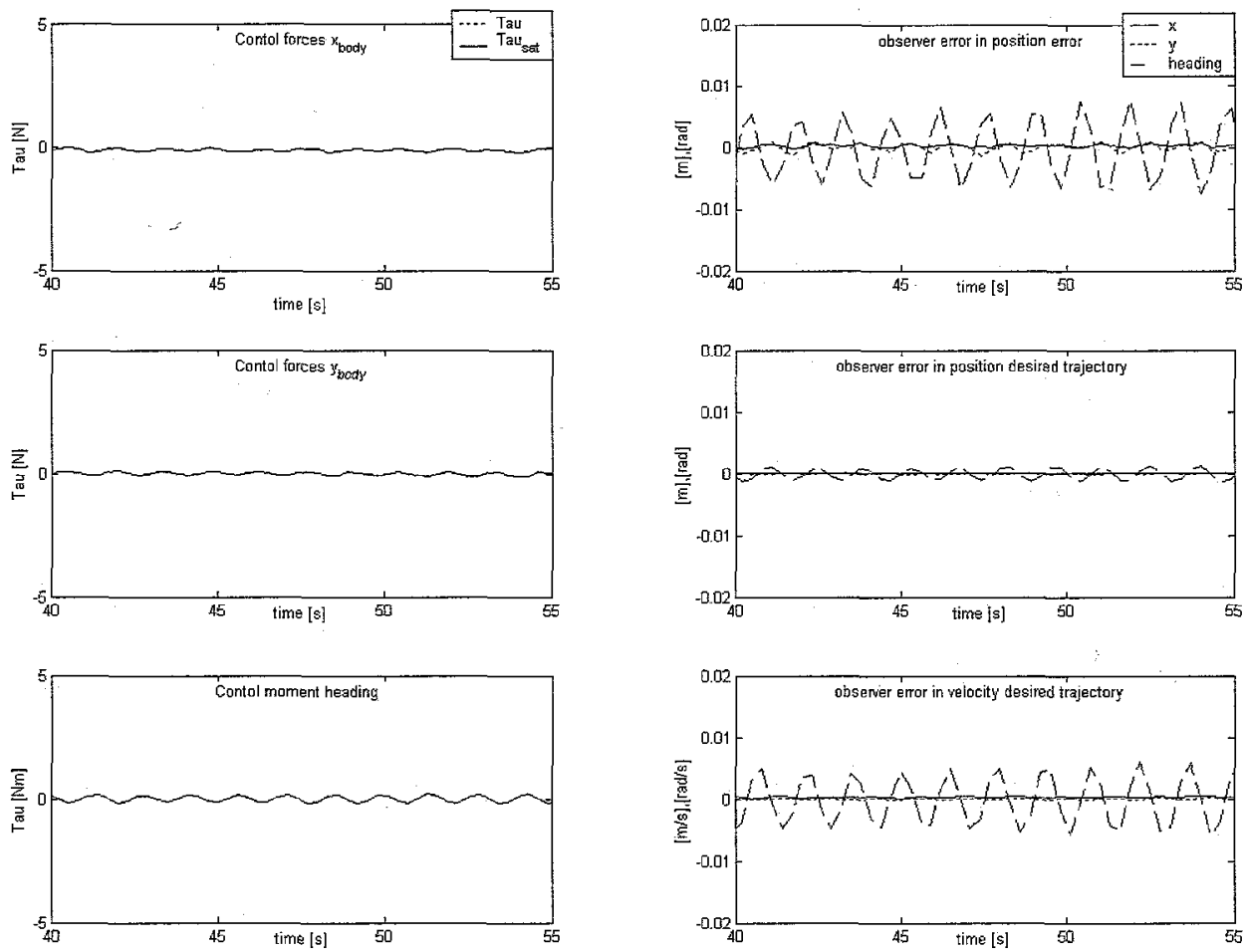

Figure 5.9 Control forces and observer errors $\widetilde{e}, \widetilde{\eta}_{d}$ and $\widetilde{\eta}_{d}$ during steady state response: gain set 2 .

In Figure 5.9 the control action and the observer errors $\widetilde{e}, \widetilde{\eta}_{d}$ and $\widetilde{\dot{\eta}}_{d}$ during the steady state response are shown. It is seen that there is much less control action necessary and the control actions are not saturated during the steady state response. This means that this controller has all the time the full control over the system. The behaviour of the ship with this set of gains is much nicer than with gain set 1 . The behaviour is smoother, errors are smaller, the control actions are smaller and especially the control actions are not saturated, which causes that the system is better in control and makes the system more predictable.

\subsection{Performance during position keeping}

To assess the performance of the system the time mean of the absolute error and the maximum of the absolute error are calculated. For practical purposes it is important to know what the maximum amplitude of the error is, because this indicates if there is a potential danger for collision. However the maximum value tells nothing about the global error, therefore the time mean of the absolute error is used (5.8). This value is derived from the IAE value (5.7) (Dorf and Bishop, 1998). This value is preferred above other values, because this value has a clear physical interpretation and is therefore more intuitive. 
$\bar{e}=\frac{1}{T} \int_{i_{o}}^{t_{0}}|e| d t$

In Table $5.5 \mathrm{a}$ and $5.5 \mathrm{~b}$ the time mean of the absolute position error and the maximum amplitude of the position error are given under different conditions. The position error is calculated in the body-fixed frame, which means that the errors can be explained as errors in the forward movement, sideward movement and heading of the ship.

The same experiment as described in section 5.3 and 5.4 is carried out under different conditions, to show the robustness of the scheme. Waves are used to disturb the system. With the DHI wave-maker system waves using the so-called JONSWAP (Joint North Sea Wave Project) distribution are used. The JONSWAP spectrum is used to describe non-fully developed seas, which means that the spectral density function will be more peaked than those representing fully developed seas. In figure 5.10 the spectral density function for the JONSWAP distribution with time mean period of $0.75 \mathrm{~s}$ and $\gamma=3.3$ is seen.

$$
T_{0}=\frac{T_{1}}{0.834}
$$

where $T_{1}$ represents the time mean period.

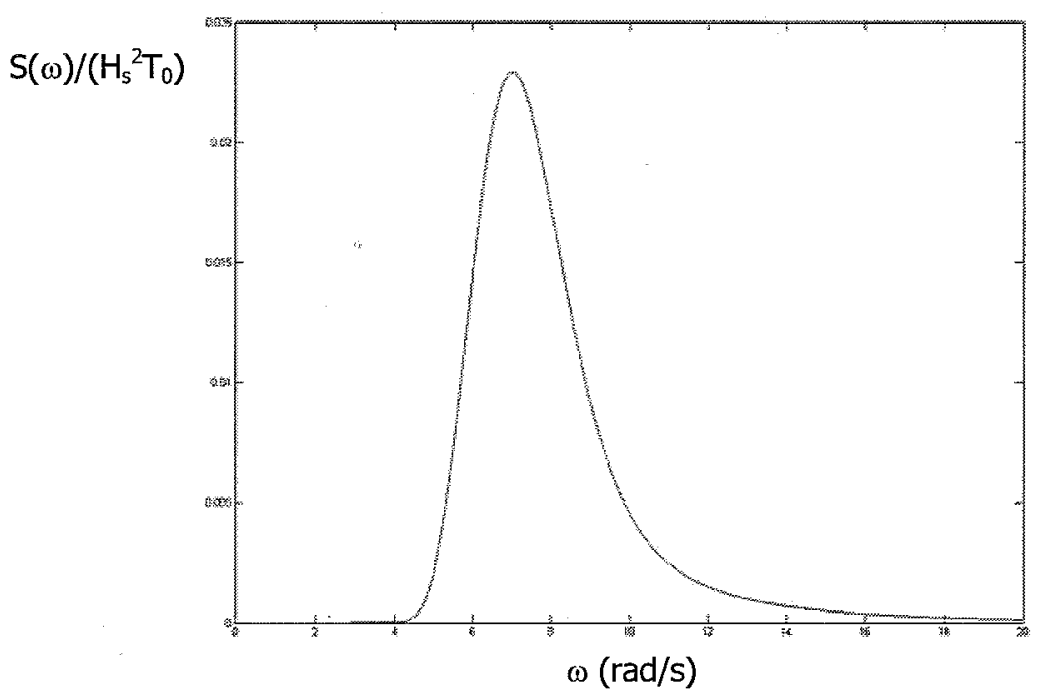

Figure 5.10 Spectral density function of the JONSWAP distribution with $\mathrm{T}_{1}=0.75 \mathrm{~s}$ and $\gamma=3.3$.

In the second experiment waves with a significant wave height of $0.01 \mathrm{~m}$ and a mean period of $0.75 \mathrm{~s}$ are used, which suits with Sea State Code 3 in full scale. Waves with a significant wave height of $0.02 \mathrm{~m}$ and a mean period of $0.75 \mathrm{~s}$ are used in the third experiment. This corresponds with Sea State Code 4 in full scale. The angle between the heading of the ship and the direction of the waves during position keeping is $180^{\circ}$, which corresponds with a head sea. This means that the experiment is done in the most ideal situation, because courses against the waves will moderate the sea effects. 


\begin{tabular}{|c|c|c|c|c|c|}
\hline \multicolumn{6}{|c|}{ Position keeping } \\
\hline \multicolumn{2}{|c|}{ x_body $[\mathrm{m}]$} & \multicolumn{2}{|c|}{ y_body $[\mathrm{m}]$} & \multicolumn{2}{|c|}{ heading [deg] } \\
\hline mean IeI & max IeI & mean IeI & $\max$ IeI & mean IeI & $\max$ IeI \\
\hline \multicolumn{6}{|c|}{ Simulation under ideal conditions } \\
\hline 0 & 0 & 0 & 0 & 0 & 0 \\
\hline \multicolumn{6}{|c|}{ Experiment without waves } \\
\hline 0.0141 & 0.0623 & 0.0125 & 0.0532 & 0.7448 & 3.5810 \\
\hline \multicolumn{6}{|c|}{ Experiment with waves ( Johnswap: $\mathrm{Hs}=0.01 \mathrm{~m}, \mathrm{Ts}=0.75 \mathrm{~s}$ ) } \\
\hline 0.0657 & 0.1406 & 0.0171 & 0.0352 & 3.2372 & 7.1276 \\
\hline \multicolumn{6}{|c|}{ Experiment with waves (Johnswap: $\mathrm{Hs}=0.02 \mathrm{~m}, \mathrm{Ts}=0.75 \mathrm{~s}$ ) } \\
\hline 0.0571 & 0.1309 & 0.0160 & 0.0402 & 3.0596 & 6.5546 \\
\hline
\end{tabular}

Table 5.5a Experimental results during position keeping: gain set 1

\begin{tabular}{|c|c|c|c|c|c|}
\hline \multicolumn{6}{|c|}{ Position keeping } \\
\hline \multicolumn{2}{|c|}{ x_body $[\mathrm{m}]$} & \multicolumn{2}{|c|}{$y_{-}$body $[\mathrm{m}]$} & \multicolumn{2}{|c|}{ heading [deg] } \\
\hline mean IeI & $\max \mathrm{IeI}$ & mean IeI & $\max I e I$ & mean IeI & $\max I e I$ \\
\hline \multicolumn{6}{|c|}{ Simulation under ideal conditions } \\
\hline 0 & 0 & 0 & 0 & 0 & 0 \\
\hline \multicolumn{6}{|c|}{ Without waves } \\
\hline 0.0068 & 0.0086 & 0.0047 & 0.0155 & 0.2865 & 0.8251 \\
\hline \multicolumn{6}{|c|}{ With waves ( Johnswap: $\mathrm{Hs}=0.01 \mathrm{~m}, \mathrm{Ts}=0.75 \mathrm{~s}$ ) } \\
\hline 0.0039 & 0.0023 & 0.0050 & 0.0060 & 0.2865 & 0.8881 \\
\hline \multicolumn{6}{|c|}{ With waves (Johnswap: $\mathrm{Hs}=0.02 \mathrm{~m}, \mathrm{Ts}=0.75 \mathrm{~s}$ ) } \\
\hline 0.0053 & 0.0099 & 0.0110 & 0.0250 & 0.4125 & 13.522 \\
\hline
\end{tabular}

During the experiments the errors increase mostly slightly with increasing disturbances, which is expected. Because the errors just slightly increase, some robustness in the scheme is shown. The errors obtained using gain set 1 are much larger than the errors obtained using gain set 2 . It is seen that during the experiments with the wave disturbance, the control action increases. This means that the control action using gain set 1 is still more saturated. This can explain the big differences in the errors in the $x-$ direction and the heading with and without waves using gain set 1 . While these big differences in the error of the $x$-direction and heading are not seen using gain set 2 . However, from gain set 2 it is known that the forces are smoother and less saturated. Here again the influence of the saturated forces is seen.

To compare these values with a full-scale operation the errors in the $x$-direction and $y$-direction have to be multiplied by the Froude number. This means that the errors from Table $5.5 \mathrm{a}$ and $5.5 \mathrm{~b}$ should be multiplied with 70 . With gain set 2 the error in the sideward movement is within 1 meter and in the forward movement the error is within 1 meter as well.

Of course the question is how comparable the full-scale situation is with the experimental set up. For example, is it possible to obtain the same measurement accuracy and same measurement frequency, is it possible for the actuators to react as fast as the actuators of Cybership II can? So a lot of remarks can be made about the compatibility of the full-scale situation and the experimental environment at the MCLab. 


\section{Chapter 6}

\section{Underway replenishment: Trajectory tracking}

In this chapter the experimental results during trajectory tracking are presented: In section 6.1 the results obtained with gain set 1 are presented and in section 6.2 the results with gain set 3 are presented. To verify the ultimately global boundedness during trajectory tracking, the experimental results are again compared with simulation results under ideal conditions. Furthermore the problems with gain set 1 are explained and how these problems are caused and solved. In section 6.3 the performance of both gain sets during trajectory tracking is assessed.

\subsection{Experimental results trajectory tracking gain set 1}

The ultimately boundedness of the synchronization system is shown during dynamical trajectory tracking. The trajectory for the main ship is defined in the Labview interface, as described in section 4.2 , by the following waypoints: [-1 0$],\left[\begin{array}{ll}-2 & 4\end{array}\right],\left[\begin{array}{ll}0 & 8\end{array}\right],\left[\begin{array}{ll}-1 & 12\end{array}\right]$ and [-1 16$]$. This trajectory can be seen in Figure 6.1. This trajectory is illustrative for a situation where the ships are given greater manoeuvrability and allows a replenishment operation in close waters. The main ship tracks this predefined path with a desired velocity of $0.2 \mathrm{~m} / \mathrm{s}$, which is equal to 3.5 knots in full scale. The main ship starts after the observer is converged. After that the supply ship is started using the button start/stop in the Labview interface. The supply ship starts in a position around $\eta_{0}=\left[-1,2,-\frac{1}{2} \pi\right]^{T}$, with initial velocity $\dot{\eta}_{0}=[0,0,0]^{T}$

In Figure 6.1 the $X Y$ plot during this experiment is given. The errors during the experiment are plotted in Figure 6.2. In Figure 6.3 the observer errors $\widetilde{e}, \widetilde{\dot{e}}, \widetilde{\eta}_{m}$ and $\widetilde{\dot{\eta}}_{m}$ are shown, while Figure 6.4 shows the control errors during the simulation under ideal conditions. In Table 6.1 the IAE values of the controller and observer errors during the experiment and simulation under ideal conditions are given. The IAE values are calculated during the first 30 seconds of the experiment and simulation. This means that the calculated IAE values correspond with the total errors in Figure 6.2, Figure 6.3 and Figure 6.4.

It is seen that the path of the supply ship depends on what point the supply ship is started with respect to the main ship. It is seen that starting the supply ship in another point with respect to the main ship, the supply ship tracks another path to the desired point. This causes some differences between the simulation results and experimentally obtained results during the transient response, because it is difficult to start the supply ship in exactly the same point with respect to the main ship. However it is seen that the experiment and simulation under ideal conditions agree.

The errors during the transient behaviour do not go to zero, but behave in a bound around zero (appendix J). This means that during trajectory tracking the experimental results and simulation results under ideal conditions seem to compare with the theoretical result of ultimately global boundedness of the synchronization closed loop errors.

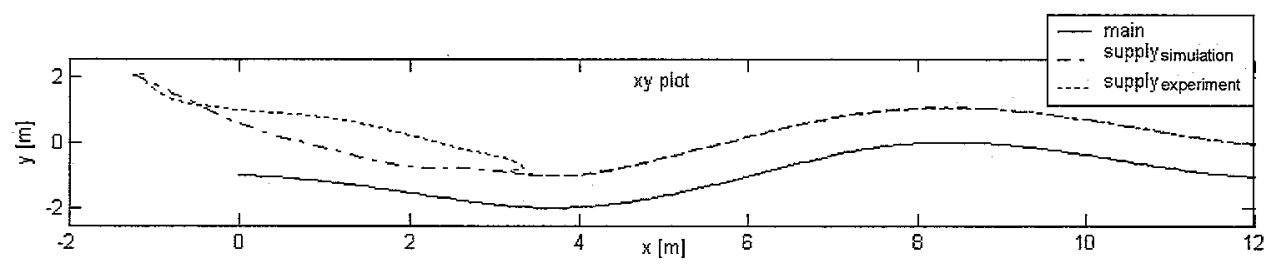

Figure 6.1 xy plot of both ships during the experiment and the simulation under ideal conditions gain set 1 . 

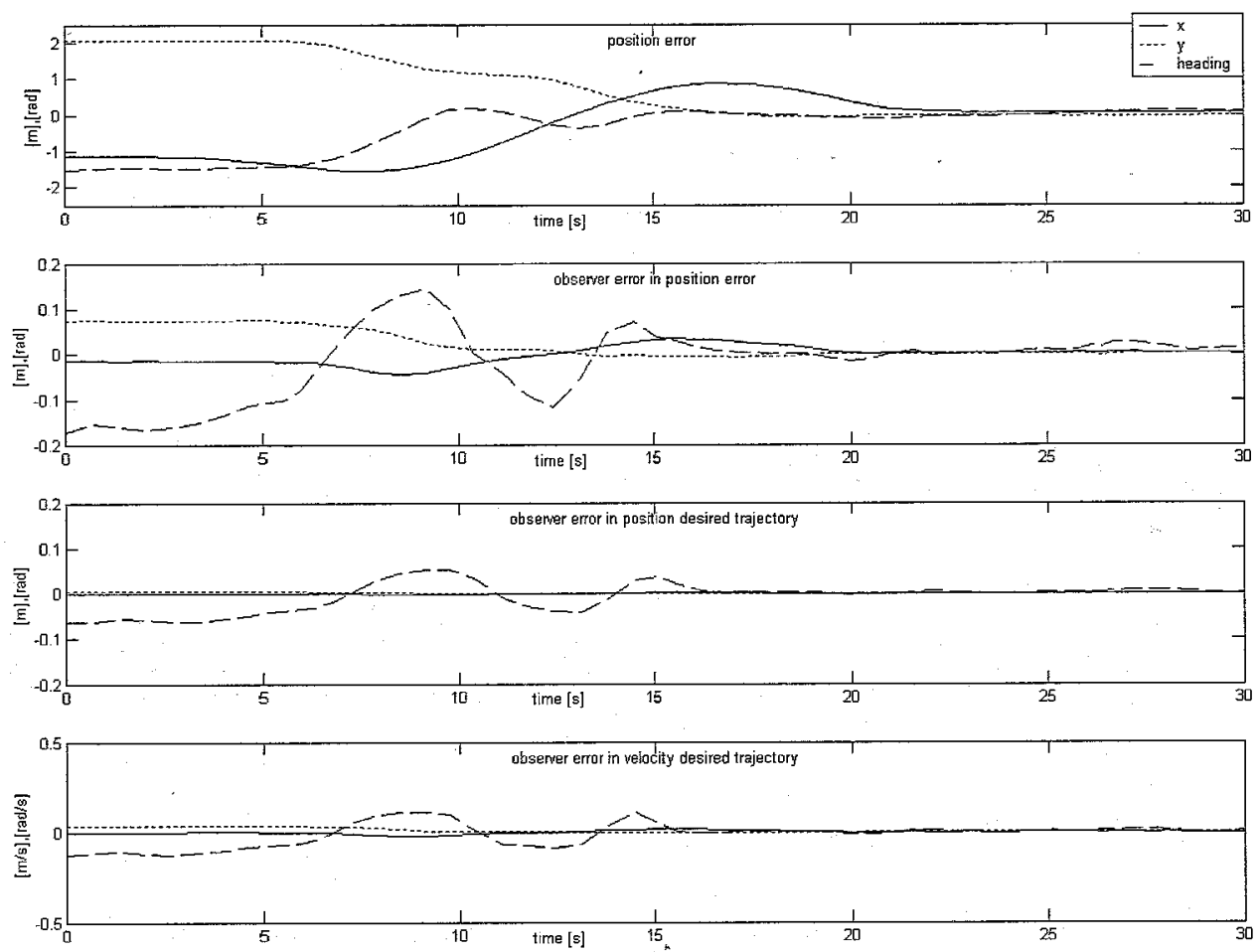

Figure 6.2 Experimental obtained $e, \widetilde{e}_{l} \widetilde{\eta}_{d}$ and ${\widetilde{\dot{\eta}_{d}}}_{d}$ during trajectory tracking: gain set 1 .
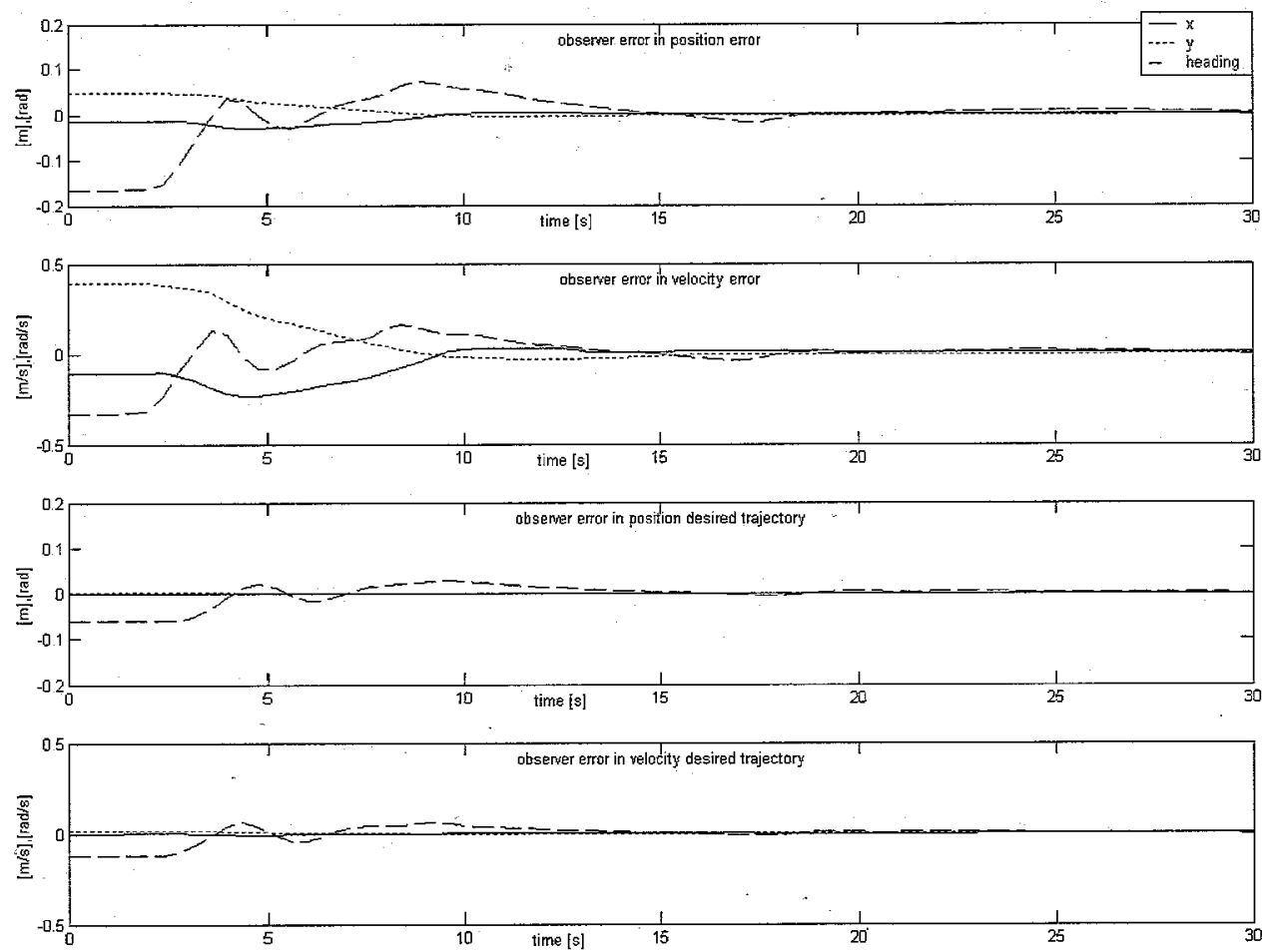

Figure 6.3 Simulation results of $\widetilde{e}_{i}, \widetilde{e}_{,} \widetilde{\eta}_{d}$ and $\widetilde{\dot{\eta}}_{d}$ during trajectory tracking: gain set 1 . 

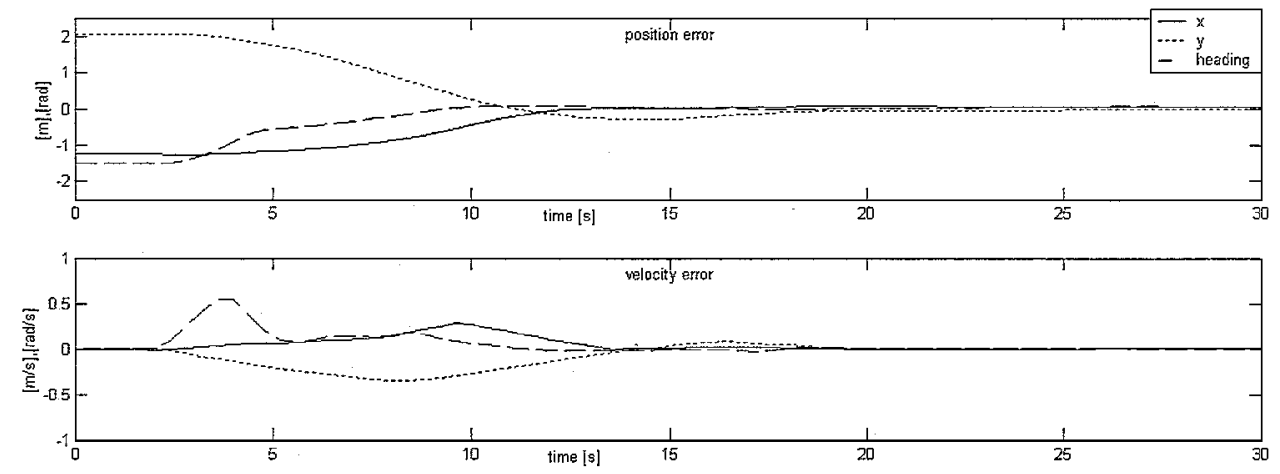

Figure 6.4 Simulation results of $e$ and $\dot{e}$ during trajectory tracking: gain set 1 .

\begin{tabular}{|l|l|l|l|l|l|l|l|l|l|l|l|l|}
\hline & IAE $e_{x}$ & IAE $e_{y}$ & IAE $e_{\varphi}$ & IAE $\widetilde{e}_{x}$ & IAE $\widetilde{e}_{y}$ & IAE $\widetilde{e}_{\varphi}$ & IAE $\tilde{\eta}_{d x}$ & IAE $\widetilde{\eta}_{d y}$ & IAE $\widetilde{\eta}_{d \varphi}$ & IAE $\tilde{\dot{\eta}}_{d x}$ & IAE $\tilde{\dot{\eta}}_{d y}$ & IAE $\tilde{\dot{\eta}}_{d \varphi}$ \\
\hline $\begin{array}{l}\text { experiment } \\
\text { simulation }\end{array}$ & 28.1696 & 14.6623 & 13.4237 & 0.4258 & 0.6848 & 1.6496 & 0.0186 & 0.0396 & 0.6754 & 0.1491 & 0.3151 & 1.3643 \\
\hline
\end{tabular}

Table 6.1 IAE values for the closed loop synchronization errors: $e, \widetilde{e}_{t} \widetilde{\eta}_{d}$ and $\widetilde{\dot{\eta}}_{d}$

In Figure 6.5 the control action during the transient response is shown. Here the saturated forces are seen again. Besides the saturation of the forces, the forces are fluctuating a lot as well. During the experiments it is seen that this causes cavitation on the screws, which causes an ineffective use of the available forces and causes a lot of noise. In addition high fluctuations in the body fixed $y$-direction can cause some roll of the ship. This is very natural because if the ship needs control action in the body fixed $y$-direction, it makes a moment with its screws and counteracts this moment with the bow thruster. To reduce the control action another set of gains is developed especially for trajectory tracking. This set will be discussed in section 6.2 .
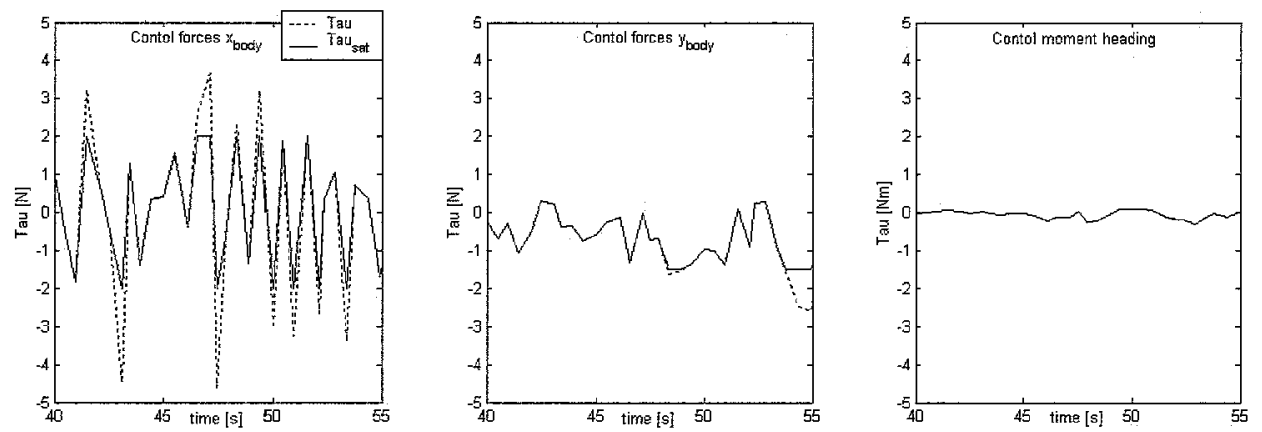

Figure 6.5 Control forces and moment during experiment: gain set 1 . 


\subsection{Experimental results trajectory tracking gain set 3}

During trajectory tracking the errors are bounded, while during position keeping there is exponential convergence of the synchronization system. To make the bound smaller during trajectory tracking, the $L_{p 2}$ gain should be chosen as high as possible. This means that the $K_{d}$ gain should be chosen as low as possible. This is in agreement what is expected, faster observers during trajectory tracking. In addition the $K_{p}$ gain should be chosen as high as possible to make the error bound as small as possible. After a lot of simulations (appendix L) the following set of gains is found especially for trajectory tracking:

Set 3:

$$
\begin{aligned}
& \mathrm{K}_{\mathrm{p}, \mathrm{b}}=\operatorname{diag}\left[\begin{array}{lll}
100 & 40 & 10
\end{array}\right] \quad \mathrm{K}_{\mathrm{d}, \mathrm{b}}=\operatorname{diag}\left[\begin{array}{lll}
30 & 20 & 5
\end{array}\right] \\
& L_{p 1}=\operatorname{diag}\left[\begin{array}{lll}
8 & 8 & 2
\end{array}\right] \quad L_{p 2}=\operatorname{diag}\left[\begin{array}{lll}
100 & 100 & 5
\end{array}\right]
\end{aligned}
$$

\begin{tabular}{|c|c|c|c|}
\hline direction & poles controller & poles observer supply ship & poles observer synchronization error \\
\hline $\mathrm{x}$ & $-0.5953+1.8766 \mathrm{i}$ & $-4,0000+9,1652 \mathrm{i}$ & $-4.5953+9.6065 \mathrm{i}$ \\
& $-0.5953-1.8766 \mathrm{i}$ & $-4,0000-9,1652 \mathrm{i}$ & $-4.5953-9.6065 \mathrm{i}$ \\
\hline $\mathrm{y}$ & $-0.3065+1.0408 \mathrm{i}$ & $-4,0000+9,1652 \mathrm{i}$ & $-4.3135+9.3589 \mathrm{i}$ \\
& $-0.3065-1.0408 \mathrm{i}$ & $-4,0000-9,1652 \mathrm{i}$ & $-4.3135-9.3589 \mathrm{i}$ \\
\hline$\varphi$ & $-1.0141+1.6292 \mathrm{i}$ & $-1,0000+2,0000 \mathrm{i}$ & $-2.0071+2.9431 \mathrm{i}$ \\
& $-1.0141-1.6292 \mathrm{i}$ & $-1,0000-2,0000 \mathrm{i}$ & $-2.0071-2.9431 \mathrm{i}$ \\
\hline
\end{tabular}

Table 6.2 Poles observer and controller gain set 3 .

The observer gains are faster than the controller gains of the linearized system around $\eta=\left[\begin{array}{lll}0 & 0 & 0\end{array}\right]^{T}, \dot{\eta}=\left[\begin{array}{lll}0 & 0 & 0\end{array}\right]^{T}$ (appendix $\mathrm{H}$ ). Only the $\varphi$ direction of the supply ship observer is a little bit slower than the controller.

The same experiment is carried out as with gain set 1. In Figure 6.6 the XY plot during this experiment is given. The errors available from the experimental data are plotted in Figure 6.7. In Figure 6.8 the observer errors $\widetilde{e}, \widetilde{\dot{e}}, \widetilde{\eta}_{m}$ and $\widetilde{\dot{\eta}}_{m}$ are plotted and in Figure 6.9 the control errors are shown during the simulation under ideal conditions.

The simulated path under ideal conditions seems not to fit with the experimental path. It seems that there is much more damping in the system during the experiment than during the simulation. This can be explained by using only the linear damping in the simulated ship during the simulation under ideal conditions, while Cybership II during the experiment is of course subject to nonlinear damping. If the experiment is compared with a simulation where the ship is simulated with nonlinear damping (appendix I), the results seem to fit well. The reason that this is so clearly seen during this experiment is because a very low $K_{d}$ gain is used. This means that the ship damping is the dominant damping in this system. However even here the experimental results support the theoretic results of ultimately global boundedness.

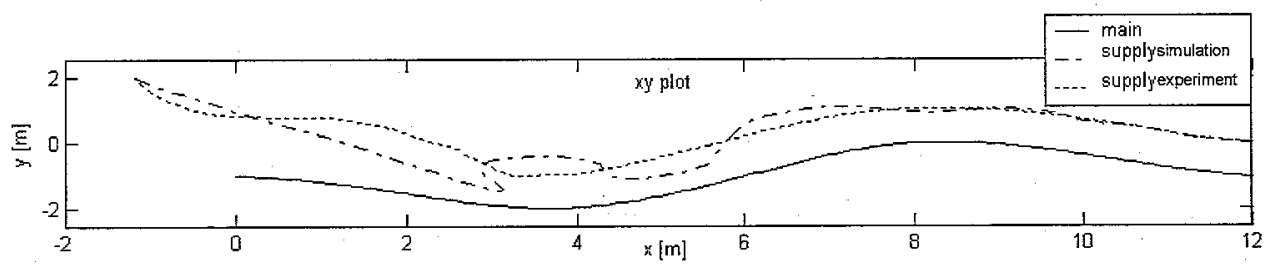

Figure $6.6 \mathrm{xy}$ plot of both ships during the experiment and the simulation under ideal conditions: gain set 3 . 

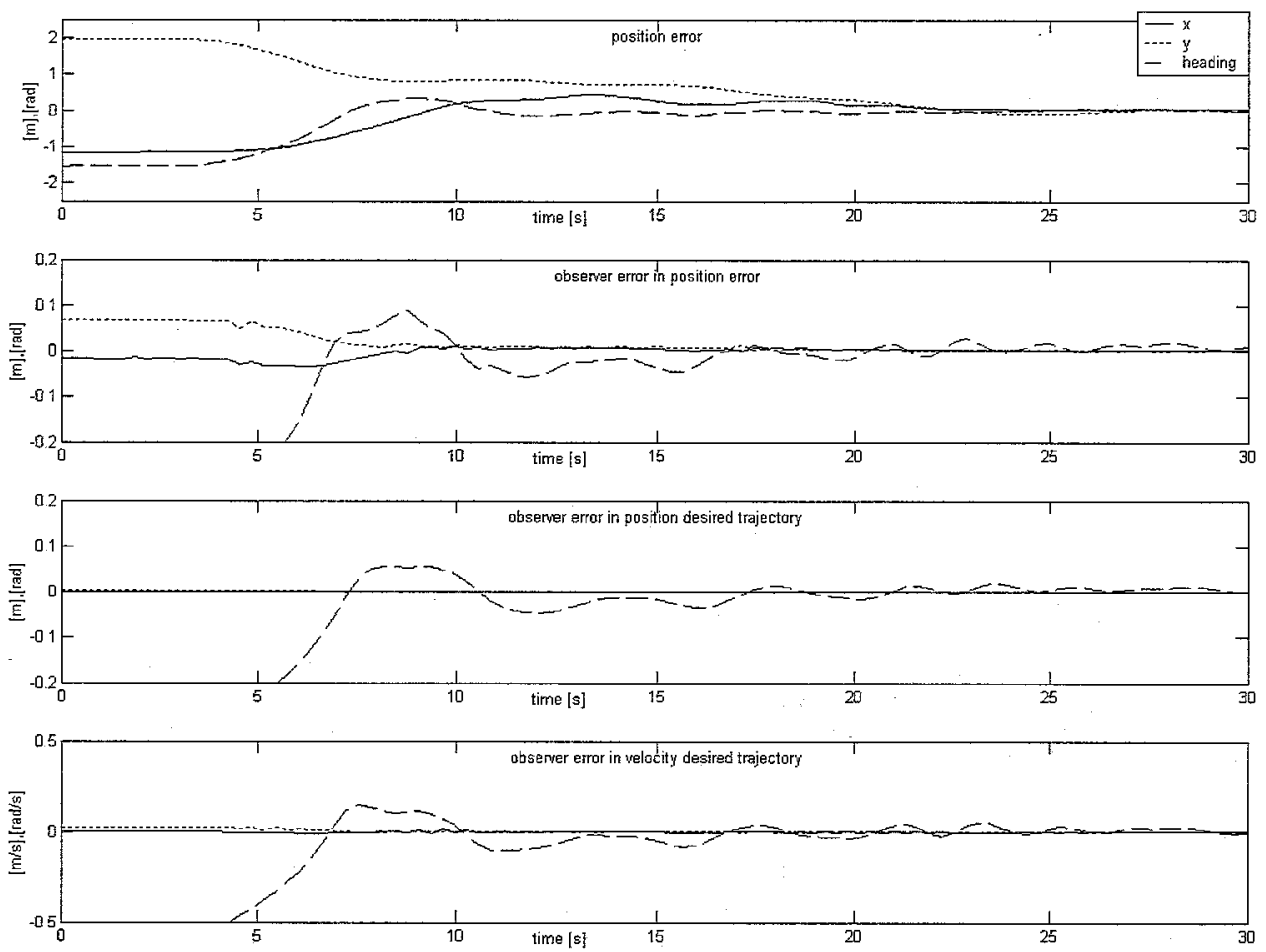

Figure 6.7 Experimental obtained $e, \widetilde{e}, \widetilde{\eta}_{d}$ and $\widetilde{\eta}_{d}$ during trajectory tracking: gain set 3 .
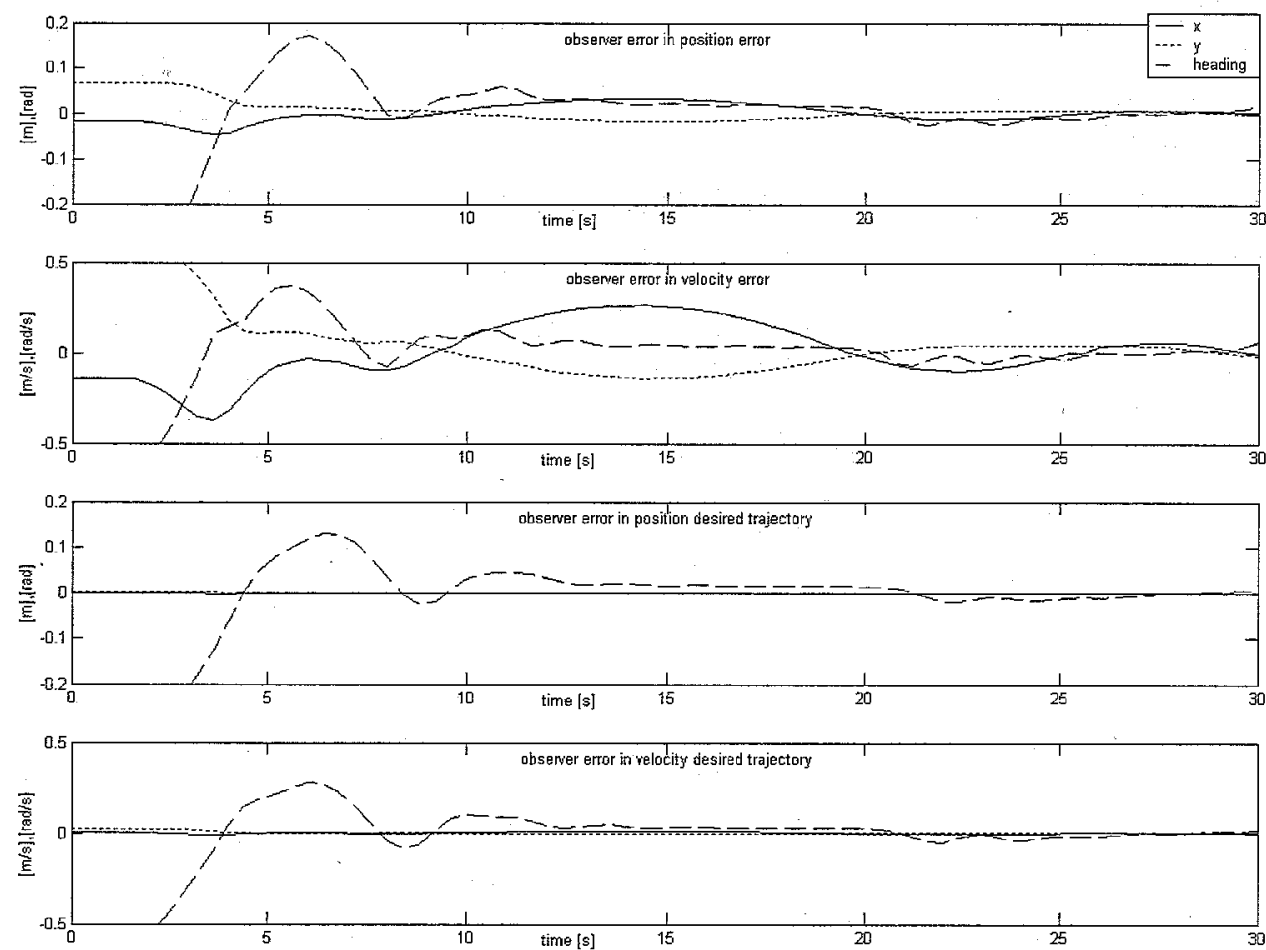

Figure 6.8 Simulation results of $\widetilde{e}, \widetilde{\dot{e}}, \widetilde{\eta}_{d}$ and $\widetilde{\dot{\eta}}_{d}$ during trajectory tracking: gain set 3 . 

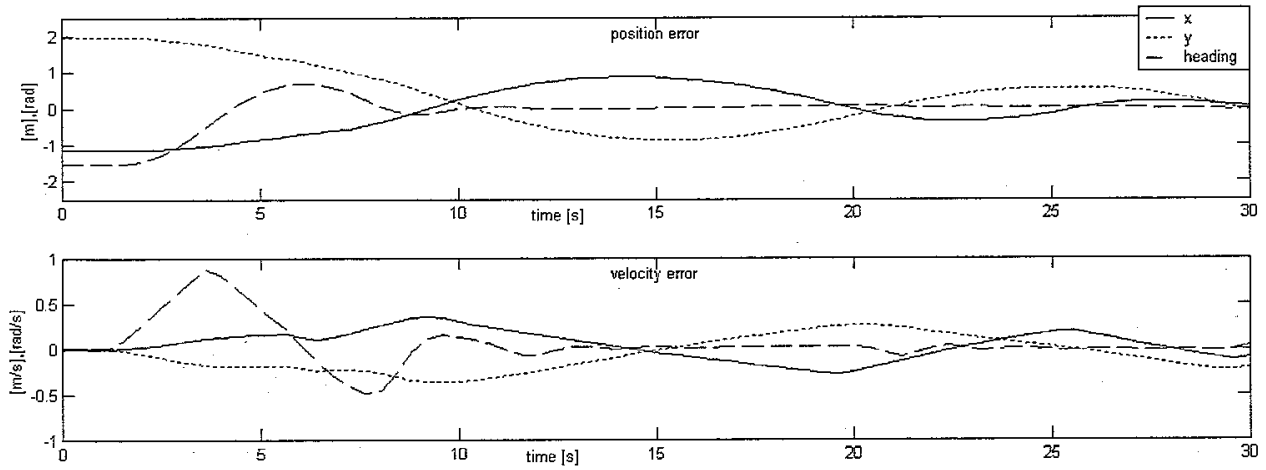

Figure 6.9 Simulation results of $e$ and $\dot{e}$ during trajectory tracking: gain set 3 .

\begin{tabular}{|c|c|c|c|c|c|c|c|c|c|c|c|c|}
\hline & $\operatorname{IAE} e_{x}$ & $\operatorname{IAE} e_{y}$ & $\operatorname{|AE} e_{\varphi}$ & IAE $\widetilde{e}_{x}$ & $\operatorname{IAE} \widetilde{e}_{y}$ & $\operatorname{IAE} \widetilde{e}_{\varphi}$ & IAE $\widetilde{\eta}_{d x}$ & $\operatorname{IAE} \widetilde{\eta}_{d y}$ & $\operatorname{IAE} \widetilde{\eta}_{d \varphi}$ & IAE $\widetilde{\dot{\eta}}_{d x}$ & IAE $\dot{\eta}_{d y}$ & IAE $\dot{\eta}_{d \varphi}$ \\
\hline & & & & 0.2517 & 0.5134 & 2.7038 & 0.0063 & 0.0183 & 2.1587 & 0.0568 & 0.1452 & 41894 \\
\hline & 19.5537 & & & & & 1 & & & 1.6596 & 0.1265 & 0.1252 & \\
\hline & & & & & & & & & & 0.0716 & & \\
\hline
\end{tabular}

Table 6.3 IAE values for the closed loop synchronization errors: $e, \widetilde{e}, \widetilde{\eta}_{d}$ and $\widetilde{\dot{\eta}}_{d}$

The most important thing is to see if the control action is smoother and lower and that the errors are in an acceptable range. In Figure 6.10 the control forces during transient behaviour is shown.
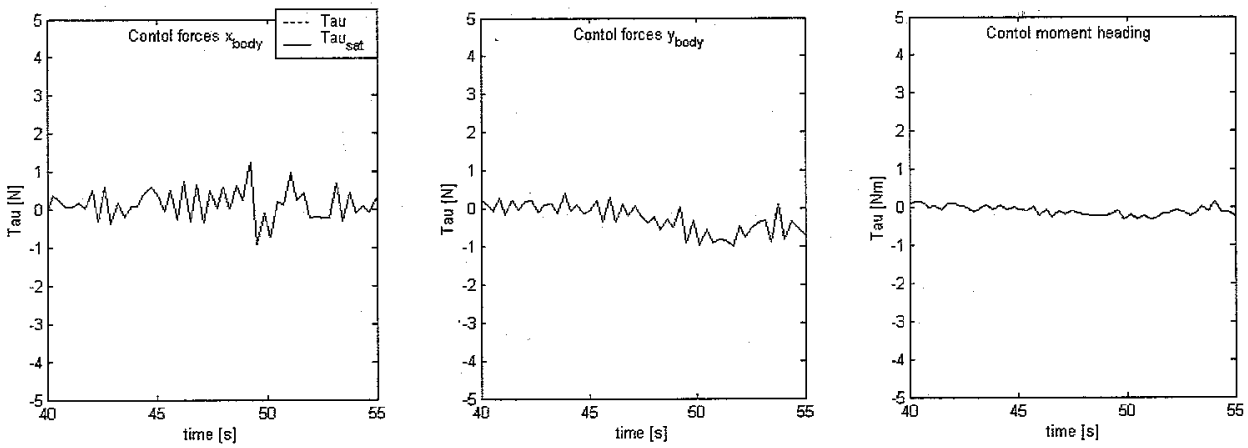

Figure 6.10 Control forces and moment during experiment: gain set 3 .

If Figure 6.10 is compared with Figure 6.5 and the mean absolute errors of gain set 1 and gain set 3 in Table $6.4 \mathrm{a}$ and Table $6.4 \mathrm{~b}$ are compared, it is seen that the control forces as well as the errors are smaller and smoother, which gives a much nicer behaviour of the ship. Thereafter the calculated control forces are not saturated, which means that the system is in full control of the observer controller system. Besides that it will not introduce errors in the observer. 


\subsection{Performance during trajectory tracking}

Table 6.4a and 6.4b show the different values during tracking the path described in the experiments above. The calculation starts at $x=2 \mathrm{~m}$ and stops at $x=10 \mathrm{~m}$, so the same part of the trajectory is used to calculated the time mean of the absolute error and the maximum of the absolute error. The disadvantage of using the same path is that if there is still no steady state behaviour at $x=2 \mathrm{~m}$, the calculated mean absolute error and maximum absolute errors give information.

The waves during the experiments are waves using the JONSWAP distribution with a significant wave height of $0.01 \mathrm{~m}$ and a mean period of $T_{\mathrm{s}}=0.75 \mathrm{~s}$. This corresponds with Sea State Code 3 in full scale, which are waves with a wave height between $0.5 \mathrm{~m}$ and $1.25 \mathrm{~m}$.

\begin{tabular}{|c|c|c|c|c|c|c|}
\hline \multicolumn{7}{|c|}{ Experiments:tracking predefined path } \\
\hline & \multicolumn{2}{|c|}{$\mathrm{x}$ body $[\mathrm{m}]$} & \multicolumn{2}{|c|}{$y \_$body $[\mathrm{m}]$} & \multicolumn{2}{|c|}{ heading [deg] } \\
\hline $\mathrm{Ud}[\mathrm{m} / \mathrm{s}]$ & mean IeI & Max IeI & mean IeI & $\max \mathrm{IeI}$ & mean IeI & $\max$ IeI \\
\hline \multirow{3}{*}{0.2} & \multicolumn{6}{|c|}{ Simulation under ideal conditions } \\
\hline & 0.0770 & 0.0969 & 0.0358 & 0.2669 & 2.1485 & 4.1768 \\
\hline & \multicolumn{6}{|c|}{ Experiment without waves } \\
\hline 0.1 & 0.0307 & 0.0515 & 0.0077 & 0.0226 & 1.4897 & 4.7154 \\
\hline 0.2 & 0.0594 & 0.0791 & 0.0177 & 0.0422 & 1.6730 & 4.4462 \\
\hline \multirow[t]{2}{*}{0.3} & 0.0753 & 0.0939 & 0.0465 & 0.1515 & 2.8533 & 8.9494 \\
\hline & \multicolumn{6}{|c|}{ With waves ( Johnswap: Hs $=0.01 \mathrm{~m}, \mathrm{Ts}=0.75 \mathrm{~s}$ ) } \\
\hline 0.1 & 0.0386 & 0.0598 & 0.0101 & 0.0273 & 1.6845 & 5.7926 \\
\hline 0.2 & 0.0606 & 0.0875 & 0.0221 & 0.0441 & 2.0798 & 6.0275 \\
\hline 0.3 & 0.0767 & 0.1001 & 0.0572 & 0.1543 & 3.3403 & 8.6975 \\
\hline
\end{tabular}

Table 6.4a Experimental results during trajectory tracking: gain set 1.

\begin{tabular}{|c|c|c|c|c|c|c|}
\hline \multicolumn{7}{|c|}{ Experiments:tracking predefined path } \\
\hline & \multicolumn{2}{|c|}{$\mathrm{x}$ body $[\mathrm{m}]$} & \multicolumn{2}{|c|}{ y_body $[\mathrm{m}]$} & \multicolumn{2}{|c|}{ heading [deg] } \\
\hline $\mathrm{Ud}[\mathrm{m} / \mathrm{s}]$ & mean IeI & Max IeI & mean IeI & $\max \mathrm{IeI}$ & mean IeI & $\max$ IeI \\
\hline \multirow{3}{*}{0.2} & \multicolumn{6}{|c|}{ Simulation under ideal conditions } \\
\hline & 0.1432 & 0.9617 & 0.2587 & 0.5785 & 1.5584 & 4.7555 \\
\hline & \multicolumn{6}{|c|}{ Simulation with nonlinear damping } \\
\hline \multirow[t]{2}{*}{0.2} & 0.0770 & 0.2189 & 0.0291 & 0.1930 & 0.7792 & 3.7299 \\
\hline & \multicolumn{6}{|c|}{ Experiments without waves } \\
\hline 0.1 & 0.0278 & 0.0421 & 0.0029 & 0.0128 & 0.4641 & 1.2490 \\
\hline 0.2 & 0.0548 & 0.0783 & 0.0123 & 0.0323 & 0.5214 & 2.4064 \\
\hline \multirow[t]{2}{*}{0.3} & 0.0790 & 0.1050 & 0.0367 & 0.0896 & 1.1860 & 3.7701 \\
\hline & \multicolumn{6}{|c|}{ With waves (Johnswap: $\mathrm{Hs}=0.01 \mathrm{~m}, \mathrm{Ts}=0.75 \mathrm{~s}$ ) } \\
\hline 0.1 & 0.0293 & 0.0503 & 0.0048 & 0.0169 & 0.4412 & 1.5126 \\
\hline 0.2 & 0.0555 & 0.0775 & 0.0146 & 0.0320 & 0.6818 & 2.2002 \\
\hline 0.3 & 0.0790 & 0.1047 & 0.0408 & 0.0969 & 1.0600 & 4.3774 \\
\hline
\end{tabular}

Table 6.4b Experimental results during trajectory tracking: gain set 3.

The general trend in the mean absolute error and the maximum absolute error is that both increase with the velocity, which is expected. If the ship tracks a distance with a higher velocity then it has relatively 
less time to compensate the errors. The advantage of a higher velocity is that the ship tracks the path smoother.

The difference between the mean and maximum absolute error in the forward direction is relatively small, which indicates that the error should be more or less constant. The positive sign of this error during the steady state behaviour (Figure 6.2, Figure6.4 and Figure 6.7) is remarkable, because a negative error will be expected. If a ship has to follow another ship, it should be natural if this ship moves behind the ship that creates the trajectory. This is remarkable but cannot be supported by using measurements that are taken one time step earlier. If the $K_{p}$ gain increases than the error becomes smaller. This is in contradiction with using position measurements that are taken one time step earlier. If measurements are used that are taken one time step earlier then the supply ship is seen behind the main ship and increasing the gain $\mathrm{K}_{\mathrm{p}}$ will increase the forward error. This supports that actual measurements are used, but of course there will be some time delay in the system.

A possible explanation for the unexpected sign of the error in the forward direction can perhaps be found in the logging of the signals. The different signals are logged in an output vector. After each position measurement the output vector is send from the onboard computer on Cybership II via the wireless network to the laptop. If the calculated signals are logged in a new output vector at once the output vector is send to the laptop, while the measured position signals of the supply ship are logged just before the output vector is send, then there is a time delay of about 0.3 seconds between the logging of the calculated signals and the measured signals. In Table $6.4 \mathrm{a}$ and Table $6.4 \mathrm{~b}$ it is seen that the mean of the absolute position error in the forward direction is approximately of the same order of the time delay multiplied with the desired forward velocity $u_{d}$. In addition this explanation can explain why the supply ship is seen in front of the main ship. However it is difficult to prove that this explanation is true, which means that the unexpected sign of the position error in the forward direction still remains an unresolved issue.
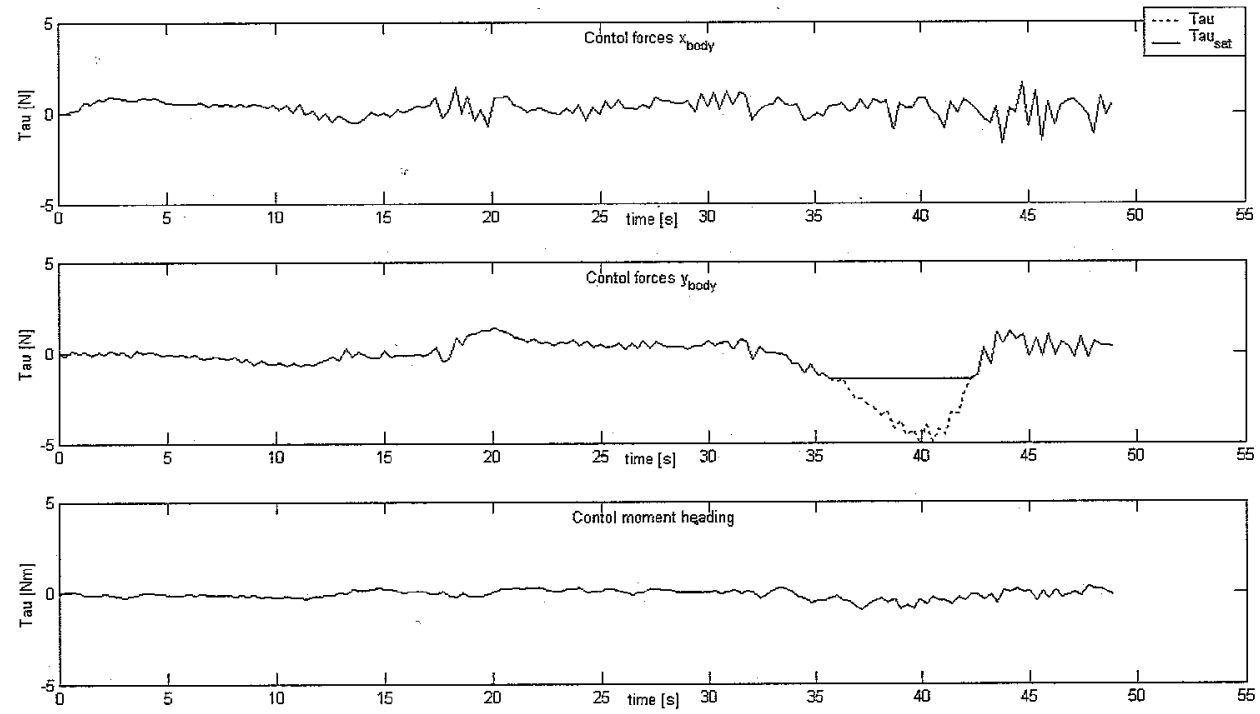

Figure 6.11 Control action during experiment trajectory tracking with gain set 3 and $u_{d}=0.3 \mathrm{~m} / \mathrm{s}$

Another remarkable error is the large error in the sideward movement and heading at $0.3 \mathrm{~m} / \mathrm{s}$. This error occurred after $x=8 \mathrm{~m}$, where the supply ship sways out the outer bend. Probably the desired trajectory for the supply vessel is out of the physical restrictions of the supply vessel. It seems that this error is not caused by bad control but because the desired trajectory is out of the physical limitations of the ship. Figure 6.11 supports this explanation, here it is seen that the control force in the body fixed y direction is saturated. This shows that the thrusters cannot generate the forces that are necessary to control the ship to the right position and it is not the observer-controller scheme, which is unable to control the ship here. 
Of course the observer-controller scheme calculates the forces, so it depends on how effective the observer-controller scheme uses the available forces. For example during trajectory tracking if gain set 1 and gain set 3 are compared, then gain set 3 uses the available forces much more effective than gain set 1.

If the errors with and without waves are compared, the robustness of the schemes can be seen. During tracking of a straight line more or less the same behaviour can be seen (appendix J). Of course the sideward movement and heading errors are less. 


\section{Conclusion and recommendations}

The theoretical results of the observer-controller scheme presented by Kyrkjebø and Pettersen (2003) during underway replenishment at sea are experimentally verified. In particular, an increased understanding of the proposed observer-controller scheme is obtained.

Using a back-to-back comparison with a simulation under ideal condition, the experimental results are found to comply well with the theoretical results of exponential convergence during position keeping and ultimately global boundedness of the synchronization system during trajectory tracking. The transient response, during dynamical positioning as well during trajectory tracking, has useful settling times and overshoot for practical purposes. During dynamical positioning the steady state response is lightly influenced by the measurement noise, which results in some oscillating behaviour of the errors around zero. If the performance under different conditions is assessed, there seems to be some robustness in the system. Robustness is necessary during rendezvous operations, because the ships will influence each other.

The influence of the different observer and controller gains on the performance of the observer controller scheme is seen during the tuning of the scheme. The influence on the performance is for each gain explained, which gives some guidelines for the tuning of the scheme in the future. However a more systematic gain tuning procedure, which satisfies the conditions given by Rodriguez-Angeles (2002) (Nijmeijer and Rodriguez-Angeles, 2003) and makes the error bound more predictable, should be adopted.

The influence of the measurement noise on the performance of the scheme is shown. Measurement noise in combination with high $K_{d}$ and high $L_{p 2}$ gain could cause large and highly fluctuating forces, which are saturated and cause cavitations on the screws. Saturation of the calculated control forces introduces errors in the observer. Besides that, it can generate instability in the closed loop system, which makes the behaviour of the system less predictable.

The observer-controller scheme seems to be robust for external disturbances, model errors and measurement noise. This is for practical purposes very important. Concluding that this observer-controller scheme is ready to use in full scale underway replenishment operations is still one bridge too far, because during the experiments there are no interactions, like Venturi effect and forces of the hoses and cables, between the main ship and supply ship, which can lead to much worse behaviour as presented here. Thereafter the question is how comparable the full-scale environment and experimental environment at the MCLab are.

Future work should concentrate on:

- Developing a systematic gain tuning procedure, which satisfies the conditions given by RodriguezAngeles (2002) (Nijmeijer and Rodriguez-Angeles, 2003) and which makes the error bound more predictable.

- The influence of the interaction effects between the main ship and the supply ship on the performance of the scheme deserve to be studied.

- Including higher order terms in the damping matrix of the observer controller scheme to further explore the properties of non-linear damping.

- Using other control strategies to aim at a more effective use of the available forces. 


\section{Bibliography}

Corneliussen, J (2003). Implementation of a Guidance System for Cybership II. MSc thesis. Norwegian University of Science and Technology. Trondheim, Norway.

Dorf, Richard C. and Robert H. Bishop (1998). Modern Control System. Addison-Wesley, 1998. Amsterdam, The Netherlands.

FAS (1999), Underway replenishment (UNREP) -navy ships. [online]http://fas.org/man/dod101/sys/ship\%unrep.htm. Rev: March 061999.

Fossen, T. I. (2002). Marine Control Systems. Guidance, Navigation and Control of Ships, Rigs and Underwater Vehicles. Marine Cybernetics ANS, 2002. Trondheim, Norway.

Kyrkjebø, E and K.Y. Pettersen (2003). Ship replenishment using synchronization control. Proc. $6^{\text {th }}$ IFAC Conference on Manoeuvring and Control of Marine Craft pp.286-291.

Lindegaard, Karl-Petter (2003), Acceleration Feedback in Dynamic Positioning, PhD thesis. Nowegian University of Science and Technology. Trondheim, Norway.

Manssouri, K. (2002), Tuning and performance of a CFT Master-Slave Robot System, internal report DCT.2002.70, Eindhoven University of Technology, Eindhoven, The Netherlands.

Nijmeijer H. and A. Rodriguez-Angeles (2003), Synchronization of mechanical systems, World Scientific Publishing Co. Pte. Ltd., 2003

NROTC (2003), Underway replenishment, [online] http//:www.nrotc.utah.edu/Nav\%20II/Underway\%20Replenishment.ppt

Rodriguez-Angeles, Alejandro (2002), Synchronization of Mechanical Systems, PhD thesis, Technische Universiteit Eindhoven. Eindhoven, The Netherlands.

Skjetne, R. T. I. Fossen and P. Kokotović (2003a). Robust output maneuvring for a class of nonlinear systems, Automatica, Submitted May 7th, 2002, accepted May 15th, 2003

Skjetne, R.,I.-A.F. Ihle and T.I. Fossen (2003b). Formation control by synchronizing multiple maneuvring systems. Proc. $6^{\text {th }}$ IFAC Conference on Manoeuvring and Control of Marine Craft pp.280-285

Sveen, D.A.(2003), Robust and Adaptive Tracking Control of Surface Vessel for Synchronization with an ROV: Practical Implementation on CyberShip II, MSc thesis. Nowegian University of Science and Technology. Trondheim, Norway. 


\section{Appendix A: Full scale replenishment, some background.}

The CONREP operations are subdivided into the FAS operations (Fuelling At Sea) and RAS operations (Replenishment At Sea). The term RAS is used for all replenishment operations except fuelling.

At the moment the (CONREP) replenishment operations are often done using the so-called Standard Tensioned REplenishment Alongside Method (STREAM). The STREAM rig (Figure1.1a and Figure 1.1b) is preferred above other connected replenishment methods, because it permits greater ship separation.

lands

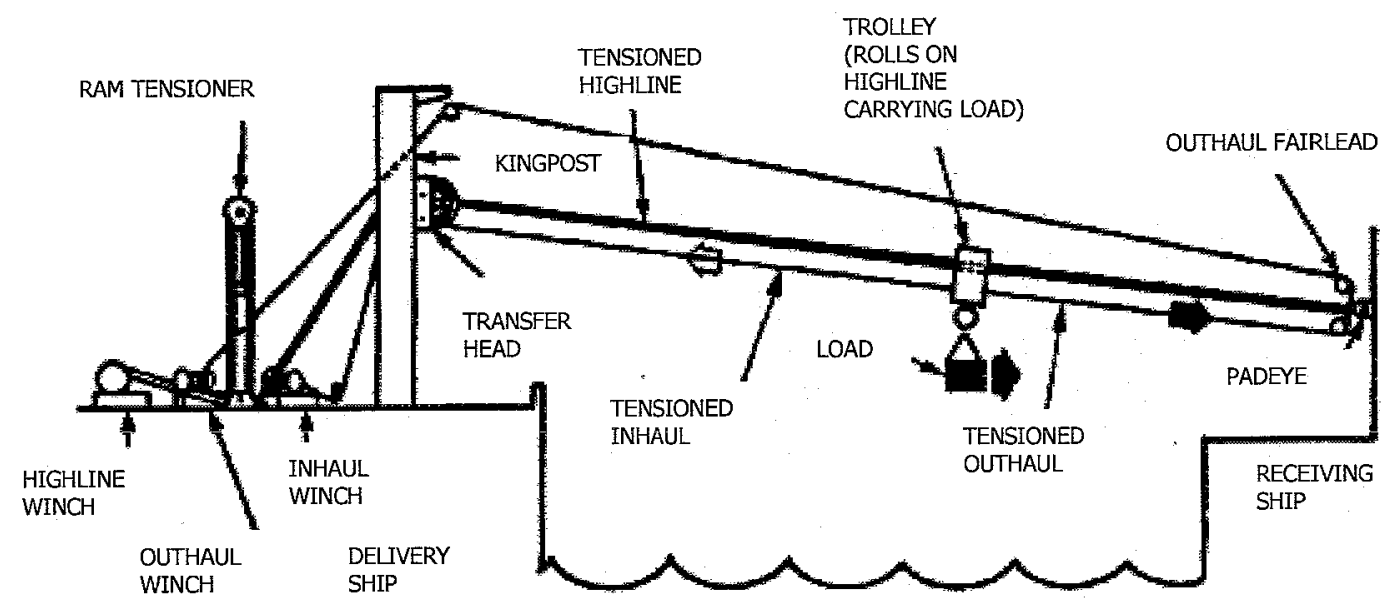

Figure 1.1a STREAM rig RAS operation.(Www.fas.org)

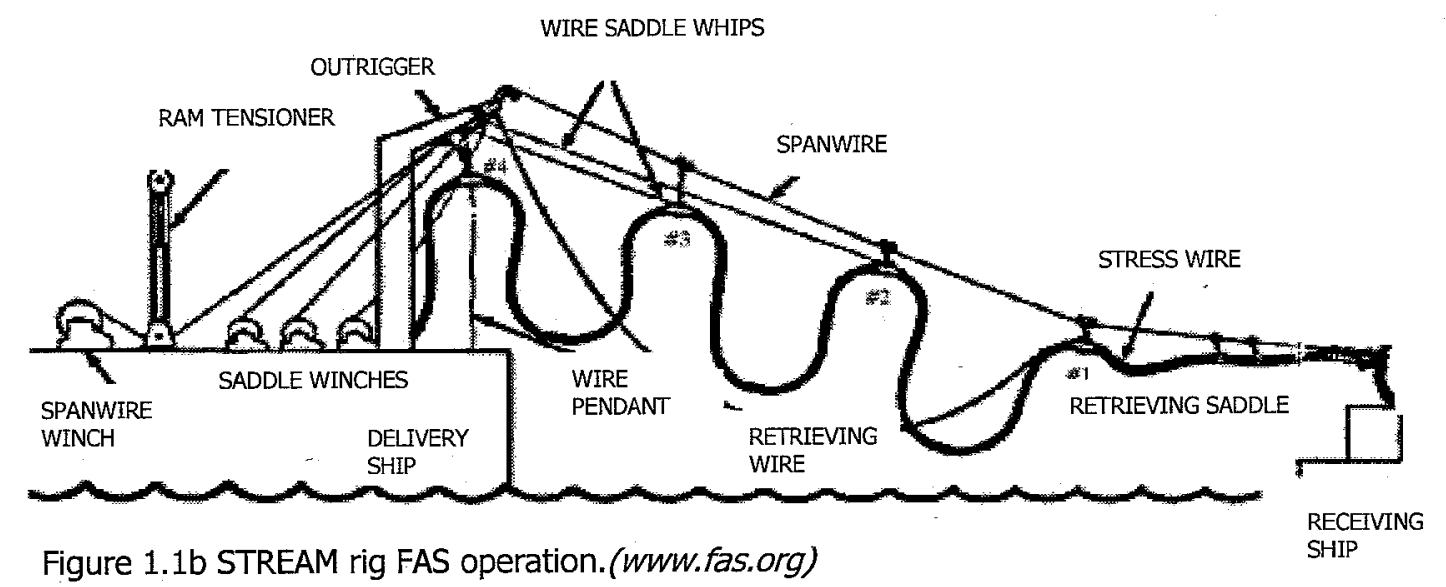

Normal speeds for ship replenishment operations are about 12-14 knots. Replenishment operations are routinely conducted at sea state code 4 , while with highly skilled personnel it can be done successfully at sea state code 5. A rule of thumb is that if the supply ship remains within 1 degree of his course, it is possible to do the replenishment operation. If the supply ship yaws within 1.5 degrees of his course it depends on experience, skill and necessity of the operation if it is a well-considered choice to start the replenishment operation. If the ship is yawing more than 2 degrees it is probably not possible to do a 
safely replenishment operation. The course of the operation depends on sea state, wind and current. Courses against the sea are preferred, because they will moderate the sea effects ( $w w w$.fas.org).

The main disturbances during replenishment operations are pressure effects. A ship underway creates an area of increased pressure at the bow and stern and an area with decreased pressure amidships. This is caused by the differences in the water flow around the hull and is known as the Venturi effect (Figure1.2). The pressure effects vary with distance between the ships, size and configuration of the ships, replenishment speed and the depth of the water. The lateral distance between the ships, depending on the sea state, expected pressure effects and the type of replenishing, is commonly between the $25 \mathrm{~m}$ and $60 \mathrm{~m}$, out to maximum $90 \mathrm{~m}$. A common approach time during replenishment operations is between the 5 and 11 minutes, while the rig and unrig time is between the 9 and 12 minutes.

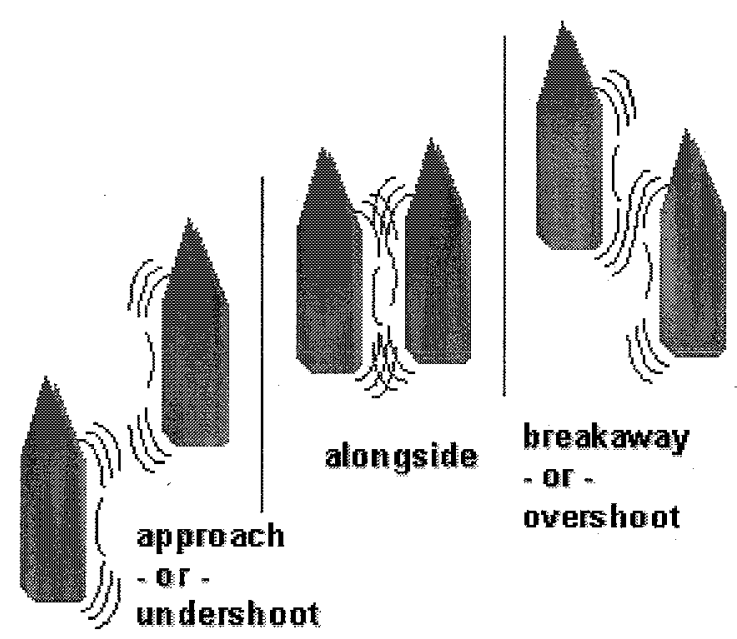

Figure 1.2 Venturi effect during underway replenishment operation.(www.nrotc.utah.edu) 


\section{Appendix B: Gain tuning procedure for observer-controller scheme.}

The following step-by-step gain tuning procedure gives a constructive way to guarantee stability and ultimate boundedness of the synchronization closed loop system, such that synchronization between the leader and follower is achieved (Rodriguez-Angeles, 2002) (Nijmeijer and Rodriguez-Angeles, 2003).

- Determine the bounds of the leader trajectories $\dot{q}_{d}, \ddot{q}_{d}$, the physical parameters $M_{s}\left(\eta_{s}\right)$, $C_{s}\left(v_{s}, \eta_{s}\right)$ and their partial derivatives with respect to $\eta_{s}$.

$$
\begin{aligned}
& \sup _{t}\left\|\dot{\eta}_{d}(t)\right\|=V_{m}<\infty \\
& \sup _{t}\left\|\ddot{\eta}_{d}(t)\right\|=A_{m}<\infty
\end{aligned}
$$

- Choose the weighting factors $\lambda_{0}>0, \varepsilon_{0}>0, \mu_{0}>0$ and $\gamma_{0}>0$ and a bound for the maximum eigenvalue of the gains $K_{p}, K_{d}, L_{p 1}$ and $L_{p 2}$, denoted by $K_{p, M}, K_{d, M}, L_{p 1, M}$ and $L_{p 2, M}$.

- The gain matrices $K_{p}, K_{d}, L_{p 1}$ and $L_{p 2}$ are chosen such that their minimum eigenvalues, denoted by $K_{p, m}, K_{d, m}, L_{p 1, m}$ and $L_{p 2, m}$, satisfy

$$
\begin{aligned}
& L_{p 2, m}>\max \left\{\mu_{0}^{2}, \gamma_{0}^{2}, L_{p 2 q 4}, L_{p 2 q 5}, L_{p 2 q 6}\right\} \\
& L_{p 1, m}>\max \left\{2 \mu_{0}, L_{p 1 q 3}, L_{p 1 q 5, a}, L_{p 1 q 5, b}\right\} \\
& K_{d, m}>\max \left\{K_{d q 1}\right\} \\
& K_{p, m}>\max \left\{K_{p q 2}, K_{p q 6}\right\}
\end{aligned}
$$

with $L_{p 2 q 4}, L_{p 2 q 5}, L_{p 2 q 6}, L_{p 1 q 3}, L_{p 1 q 5, a}, L_{p 1 q 5, b}, K_{d q 1}, K_{p q 2}$ and $K_{p q 6}$ given by

$$
-K_{d q 1}=\lambda_{0} M_{s, M}
$$

$-K_{p q 2}=\frac{\lambda_{0} V_{M}\left(M_{s, p M}-C_{s, M}\right)^{2}}{4\left(K_{d, m}-\lambda_{0} M_{s, M}\right)}$

- $L_{p 1 q 3}$ : denotes the solution of the equation $\Delta Q_{3}=a_{1} L_{p 1 q 3}+a_{2}=0$, with $a_{1}, a_{2}$ the resultant coefficients in the factorization of $L_{p 1, m}$ in $\Delta Q_{3}$, and $L_{p 1, m}$ substituted by $L_{p 1 q 3}$. 
- $L_{p 2 q 4}$ : denotes the solution of the equation $\Delta Q_{4}=b_{1} L_{p 2 q 4}+b_{2}=0$, with $b_{1}, b_{2}$ the resultant coefficients in the factorization of $L_{p 2, m}$ in $\Delta Q_{4}$, and $L_{p 2, m}$ substituted by $L_{p 2 q 4}$.

$-L_{p 1 q 5, \alpha}=2\left(\gamma_{0}-C_{s, M} V_{M} M_{s, M}{ }^{-1}\right)$

- $L_{p 1 q 5, b}$ : denotes the largest solution of the equation $\Delta Q_{5}=c_{0}+c_{1} L_{p 1 q 5}+c_{2} L_{p 1 q 5}{ }^{2}=0$, with $c_{0}, c_{1}, c_{2}$ the resultant coefficients in the factorization of $L_{p 1, m}$ in $\Delta Q_{5}$, and $L_{p 1, m}$ substituted by $L_{p 1 q 5}$.

- $L_{p 2 q 5}$ : denotes the solution of the equation $c_{2}=r_{1} L_{p 2 q 5}+r_{2}=0$, with $c_{2}$ as in $L_{p 1 q 5, b}, r_{1}, r_{2}$ the resultant coefficients in the factorization of $L_{p 2, m}$ in $c_{2}$, and $L_{p 2, m}$ substituted by $L_{p 2 q 5}$.

- $L_{p 2 q 6}$ : denotes the largest solution of the equation $\Delta Q_{6}=t_{0}+t_{1} L_{p 2 q 6}+t_{2} L_{p 2 q 6}{ }^{2}=0$, with $t_{0}, t_{1}, t_{2}$ the resultant coefficients in the factorization of $L_{p 2, m}$ in $\Delta Q_{6}$, and $L_{p 2, m}$ substituted by $L_{p 2 q 6}$.

- $K_{p q 6}$ : denotes the solution of the equation $t_{2}=s_{1} K_{p q 6}+s_{2}=0$, with $t_{2}$ as in $L_{p 2 q 6}, s_{1}, s_{2}$ the resultant coefficients in the factorization of $K_{p, m}$ in $t_{2}$, and $K_{p, m}$ substituted by $K_{p q 6}$. 


\section{Appendix C: Screw and rudder models.}

The screw and rudder models are developed by Karl Peter Lindegraad (Lindegraad, K.-P., 2003) by measuring forces due to actuator setpoints on Cybership II, while it is moored to the basin edge. The models are assumed to be low speed models.

$$
\begin{array}{|c|c|}
\hline \mathrm{i} & \text { screw } \\
\hline 1 & \text { left screw/rudder pair } \\
2 & \text { right screw/rudder pair } \\
3 & \text { tunnel thruster } \\
\hline
\end{array}
$$

Table D.1 Actuators onboard Cybership II

For fixed pitch screws the generated thrust force is more or less proportional to the square of the screw shaft speed $\omega_{i}$. The low speed screw/rudder model for CSII can conveniently be separated into two parts: The first one is the nominal thrust (rudder angles $\delta_{i}=0, i=1,2$ )

$$
T_{i}=\left\{\begin{array}{cl}
k_{i T p} \omega_{i}^{2} & \omega_{i} \geq 0 \\
k_{i T n}\left|\omega_{i}\right| \omega_{i} & \omega_{i} \leq 0
\end{array} \quad i \in[1,3]\right.
$$

and the second part gives additional rudder lift and drag forces, $i=1,2$,

$$
\begin{aligned}
& L_{i}=\left\{\begin{array}{cc}
T_{i}\left(1+k_{i L \omega} \omega_{i}\right)\left(k_{i L \delta 1}+k_{i L \delta 2}\left|\delta_{i}\right|\right) \delta i & \omega_{i} \geq 0 \\
0 & \omega_{i} \leq 0
\end{array}\right. \\
& D_{i}=\left\{\begin{array}{cc}
T_{i}\left(1+k_{i D \omega} \omega_{i}\right)\left(k_{i D \delta 1}\left|\delta_{i}\right|+k_{i D \delta 2} \delta_{i}^{2}\right) & \omega_{i} \geq 0 \\
0 & \omega_{i} \leq 0
\end{array}\right.
\end{aligned}
$$

For the main screws, $i=1,2$, the resulting surge and sway forces are

$$
u_{i}=\left[\begin{array}{c}
T_{i}-D_{i} \\
L_{i}
\end{array}\right]
$$

\begin{tabular}{|c|c|}
\hline Parameter & Value [Ns2] \\
\hline $\mathrm{k}_{1 \mathrm{Tp},} \mathrm{k}_{2 \mathrm{~T} p}$ & $3.7410^{-3}$ \\
$\mathrm{k}_{1 \mathrm{Tn},} \mathrm{k}_{2 \mathrm{Tn}}$ & $5.0510^{-3}$ \\
$\mathrm{k}_{3 \mathrm{~T}}$ & $1.8410^{-4}$ \\
$\mathrm{k}_{3 \mathrm{~T}}$ & $1.8810^{-4}$ \\
\hline
\end{tabular}

Table D.2 Nominal thrust parameters.

\begin{tabular}{|l|l|l|}
\hline Parameter & Value & Unit \\
\hline $\mathrm{k}_{1 \mathrm{Ln}}, \mathrm{K}_{2 \mathrm{Ln}}$ & $2.1010^{-2}$ & $\mathrm{~S}$ \\
$\mathrm{~K}_{1 \mathrm{~L}} \delta_{1}, \mathrm{~K}_{2 \mathrm{~L}} \delta_{1}$ & 0.927 & $\mathrm{rad}^{-1}$ \\
$\mathrm{~K}_{1 \mathrm{~L}} \delta_{2}, \mathrm{~K}_{2 \mathrm{~L}} \delta_{2}$ & -0.5576 & $\mathrm{rad}^{-2}$ \\
$\mathrm{~K}_{1 \mathrm{Dn}}, \mathrm{K}_{2 \mathrm{Dn}}$ & $9.6410^{-3}$ & $\mathrm{~s}$ \\
$\mathrm{~K}_{1 \mathrm{D}} \delta_{1,}, \mathrm{~K}_{2 \mathrm{D}} \delta_{2}$ & 0.079 & $\mathrm{rad}^{-1}$ \\
$\mathrm{k}_{1 \mathrm{D}} \delta_{2,} \mathrm{~K}_{2 \mathrm{D}} \delta_{2}$ & 0.615 & $\mathrm{rad}^{-2}$ \\
\hline
\end{tabular}

Table D.3 Rudder lift and drag parameters. 


\section{Appendix D: List of errors, problems and possible solutions during the simulations and experiments at the MCLab.}

During the simulations and experiments different error messages occurs.

Error:

Could not send command to Opal daemon.

Possible cause:

- node with IP address 129.241 .187 .62 is not alive

- the RT-LAB daemon is not running

Solution:

The problem is most definitely with the QNX computer, which has to be restarted. The loginname is "root". Check by using the ping command "ping 129.241.10.1" that you get contact with the network. To get access to the room a PhD key is needed.

Error:

Target node " kybpc149" (IP address 129.241.187.62) is not responding. verify that the node is up and is connected to the network.

Solution:

Use "Configuration" in the RT-LAB Main Control window and choose "Advanced". There you choose "Hardware configuration" and make sure that "kybpc149" is chosen as development node. If you have to change this the password on the Dell Altitude 800 laptop is "Kristin" and on the Dell Inspiron 8200 it is "CS2". In the RT-LAB Main Control window, choose "Assign Nodes" and make sure "kybpc149" is the assigned physical node. Select "kybpc149" and try to "ping" to make sure that there is connection.

Error:

Could not complete FTP connection to address 129.241 .187 .62 . Verify your FTP user ID and password on this target.

Solution:

Use "Configuration" in the RT-LAB Main Control window and choose "Advanced". There you choose "Hardware configuration" and make sure that FTPuser ID is set as "ntuser" and FTPuserPassword is set as "ntuser" as well.

Otherwise restart the laptop and the QNX computer as well.

Error:

Watchdog error is stopping simulation.

Solution:

The systems at Gløshaugen and Tyholt are not exactly the same. The wireless network at Tyholt can handle fewer signals than the network at Gløshaugen. The solution is to reduce the "logging signals" or decrease the basic step size and/or the maximum number of samples per signal.

Error:

Buffer overflow appears sometimes at the PPU monitor.

Solution:

Switch off the measurements in the NyPos program and restart the PPU. Initialize the measurements and restart them after the PPU is restarted. 
Error:

A lot of measurements are lost.

Solution:

This might be an indication that the batteries of the active responders are empty. So they have to be changed.

Error:

During the experiments there is no communication with Cybership II and Cybership II is out of control.

Solution:

This can be dangerous because it is not possible to use the emergency control. The first thing you must do is save Cybership II to prevent it for a fatal collision with the bridge. Make sure that the battery is not empty; because this can caused the communication lose. Then restart the QNX computer on board Cybership II. Often it is necessary to restart the laptop as well. 


\section{Appendix E: IP address and password.}

IP address and passwords:

$\begin{array}{llll}\text { Gløshaugen: } & \text { IP address } & \text { Username } & \text { Password } \\ \text { QNX computer } & 129.241 .187 .62 & & \text { root }\end{array}$

Tyholt:

Laptop

Cybership II

NyPos computer

Black computer in corner
192.168.0.1

192.168 .0 .2

192.168.0.3 mcpos

mclab mcl123 (third character is a $\mathrm{L}$ ) mol123 (third character is $a \mathrm{~L}$ ) 


\section{Appendix F: Influence of the different observer and controller gains.}

\begin{tabular}{|c|c|c|c|c|c|c|c|c|c|}
\hline \multicolumn{4}{|c|}{ Observer and controller gains } & \multicolumn{6}{|c|}{ Maximum absolute errors } \\
\hline $\mathrm{L}_{p 1}$ & $\mathrm{~L}_{\mathrm{D} 2}$ & $K_{p}$ & $K_{d}$ & mean $|e|$ & mean $|\dot{e}|$ & mean $|\widetilde{e}|$ & mean $|\tilde{e}|$ & mean $\left|\widetilde{\eta}_{d}\right|$ & mean $\left|\widetilde{\dot{\eta}}_{d}\right|$ \\
\hline 682 & 1001005 & 150355 & 2007010 & 0.0483 & $8.25 \mathrm{E}-05$ & 1.80E-03 & 0.011 & $1.05 \mathrm{E}-04$ & $6.29 \mathrm{E}-04$ \\
\hline 882 & 1001005 & 150355 & 2007010 & 0.0531 & 7.05E-05 & $1.80 \mathrm{E}-03$ & 0.0147 & $1.05 \mathrm{E}-04$ & 8.37E-04 \\
\hline 1082 & 1001005 & 150355 & 2007010 & 0.0579 & 7.70E-05 & $1.80 \mathrm{E}-03$ & 0.0183 & $1.05 \mathrm{E}-04$ & $1.00 \mathrm{E}-03$ \\
\hline 682 & 1001005 & 150355 & 2007010 & 0.0467 & & & 0.0113 & $2.01 \mathrm{E}-04$ & $2.20 \mathrm{E}-03$ \\
\hline 882 & 1001005 & 150355 & 2007010 & 0.0524 & $E-03$ & 1.70 & & $E-04$ & $10 \mathrm{E}-03$ \\
\hline 1022 & 1001005 & 150355 & 2007010 & 0.0576 & 2.10E-03 & $2.10 \mathrm{E}-03$ & 0.0179 & $9.67 E-05$ & $1.00 \mathrm{E}-03$ \\
\hline 882 & 501005 & 150355 & 2007010 & & & & & $3.95 \mathrm{E}-04$ & $3.20 \mathrm{E}-03$ \\
\hline 882 & 1001005 & 150355 & 2007010 & 0.0531 & $=05$ & $1.80 \mathrm{E}-03$ & 147 & $=-04$ & 8.37E-04 \\
\hline 882 & 1501005 & 150355 & 2007010 & 0.0463 & 0.0042 & 0.0013 & 0.0099 & $4.55 \mathrm{E}-04$ & 0.0057 \\
\hline 882 & 501005 & 150355 & 2007010 & .07 & & & & $3.92 \mathrm{E}-04$ & $3.10 \mathrm{E}-03$ \\
\hline 882 & 1001005 & 150355 & 2007010 & 0.0524 & -03 & 1.7 & 154 & -04 & $1.10 \mathrm{E}-03$ \\
\hline 882 & 1501005 & 150355 & 2007010 & 0.0449 & .0034 & 0.0013 & 0.0131 & $2.55 E-04$ & 0.0032 \\
\hline 882 & 1001005 & 75355 & 200 & & & & & & $23 E-04$ \\
\hline 882 & 1001005 & 150355 & 2007010 & 0.0531 & $=-05$ & 1.8 & & -04 & 8.37E-04 \\
\hline 882 & 1001005 & 200355 & 2007010 & 0.0395 & $5.35 \mathrm{E}-05$ & 0.0018 & 0.0144 & $1.38 \mathrm{E}-04$ & 0.0011 \\
\hline 882 & 1001005 & 75355 & 2007010 & & & & & $=-05$ & $.09 \mathrm{E}-04$ \\
\hline 882 & 1001005 & 150355 & 2007010 & 0.0524 & $\mathrm{E}-03$ & $1.70 \mathrm{E}-03$ & 0.0154 & $=-04$ & $1.10 \mathrm{E}-03$ \\
\hline 882 & 1001005 & 200355 & 2007010 & 0.0392 & 0.0038 & 0.0016 & 0.015 & $1.64 \mathrm{E}-04$ & 0.0016 \\
\hline 882 & 1001005 & 150355 & 100 & & & & & & E-04 \\
\hline 882 & 1001005 & 150355 & 2007010 & 0.0531 & & 1.8 & & -04 & 8.37E-04 \\
\hline 882 & 1001005 & 150355 & 2507010 & 0.058 & 7.71E-05 & 0.0018 & 0.0147 & $1.05 \mathrm{E}-04$ & $8.38 \mathrm{E}-04$ \\
\hline 882 & 1001005 & 150355 & 1007010 & & & & & & DE-03 \\
\hline 882 & 1001005 & 150355 & 2007010 & 0.0524 & $E-03$ & $1.70 \mathrm{E}-03$ & 0.0154 & 1.27 & $1.10 \mathrm{E}-03$ \\
\hline 882 & 1001005 & 150355 & 2507010 & 0.0573 & 0.0042 & 0.0017 & 0.0146 & $1.18 \mathrm{E}-04$ & 0.001 \\
\hline
\end{tabular}

Table G.1 Influence of the different observer and controller gains, simulation with measurement noise 


\section{Appendix G: Linearized observer-controller scheme.}

A rule of thumb in control engineering is that the observer is faster than the controller. Since the system is nonlinear it is not possible to verify this directly. Therefore the system is linearized around the origin: $\eta=\left[\begin{array}{lll}0 & 0 & 0\end{array}\right]^{T}, \dot{\eta}=\left[\begin{array}{lll}0 & 0 & 0\end{array}\right]^{T}$, which results in:

$M^{*}(\eta) \ddot{\eta}+D^{*}(\eta) \dot{\eta}=\tau_{\eta}$

with $M^{*}(\eta)$ and $D^{*}(\eta)$ defined as:

$M^{*}(\eta)=R(\varphi) M R(\varphi)^{T}$
$D^{*}(\eta)=R(\varphi) D R(\varphi)^{T}$

$$
M=\left[\begin{array}{ccc}
25,8 & 0 & 0 \\
0 & 33,8 & 1,0115 \\
0 & 1,0115 & 2,76
\end{array}\right] \quad D=\left[\begin{array}{ccc}
0,72 & 0 & 0 \\
0 & 0,86 & -0,11 \\
0 & -0,11 & 0,5
\end{array}\right]
$$

Assumed is that the observer works perfect, which means that the estimated values are without errors. In addition it is assumed that the linearized dynamic model of the ship is perfect, which means that there is no unmodelled dynamics. This results in the following closed loop equation:

$$
\begin{aligned}
& M^{*}\left(\eta_{s}\right) \ddot{\eta}_{s}+D^{*}\left(\eta_{s}\right) \dot{\eta}_{s}=M^{*}\left(\eta_{s}\right) \ddot{\eta}_{d}+D^{*}\left(\eta_{s}\right) \dot{\eta}_{d}-K_{d} \dot{e}-K_{p} e \\
& M^{*}\left(\eta_{s}\right) \ddot{e}+\left(D^{*}\left(\eta_{s}\right)+K_{d}\right) \dot{e}+K_{p} e=0
\end{aligned}
$$

which can be written in the state space notation:

$$
\left[\begin{array}{c}
\dot{e} \\
\ddot{e}
\end{array}\right]=\left[\begin{array}{cc}
0 & 1 \\
-M^{*}\left(\eta_{s}\right)^{-1} K_{p} & -M^{*}\left(\eta_{s}\right)^{-1}\left(D^{*}\left(\eta_{s}\right)+K_{d}\right)
\end{array}\right]\left[\begin{array}{l}
e \\
\dot{e}
\end{array}\right]+\left[\begin{array}{l}
0 \\
0
\end{array}\right] u
$$

The synchronization error observer can be written in the state space notation as follows:

$\frac{d}{d t}\left[\begin{array}{c}\hat{e} \\ \hat{e}\end{array}\right]=\left[\begin{array}{cc}-L_{p 1} & 1 \\ -M^{*}\left(\eta_{s}\right)^{-1} K_{p}-L_{p 2} & -M^{*}\left(\eta_{s}\right)^{-1}\left(D^{*}\left(\eta_{s}\right)+K_{d}\right)\end{array}\right]\left[\begin{array}{l}\hat{e} \\ \hat{\dot{e}}\end{array}\right]+\left[\begin{array}{c}L_{p 1} \\ L_{p 2}\end{array}\right] e$

Also the supply ship observer can be written in the state space notation:

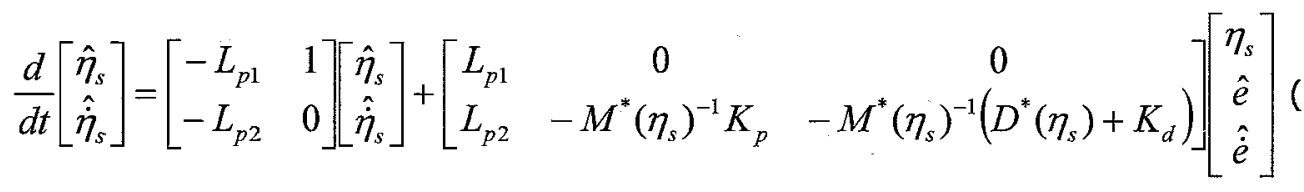

Using this linearized system, (H.6), (H.7) and (H.8), the controller and observer poles can be calculated in Matlab. 


\section{Appendix H: Figures position keeping gain set 1 and set 2.}

Experiment position keeping gain set 1:
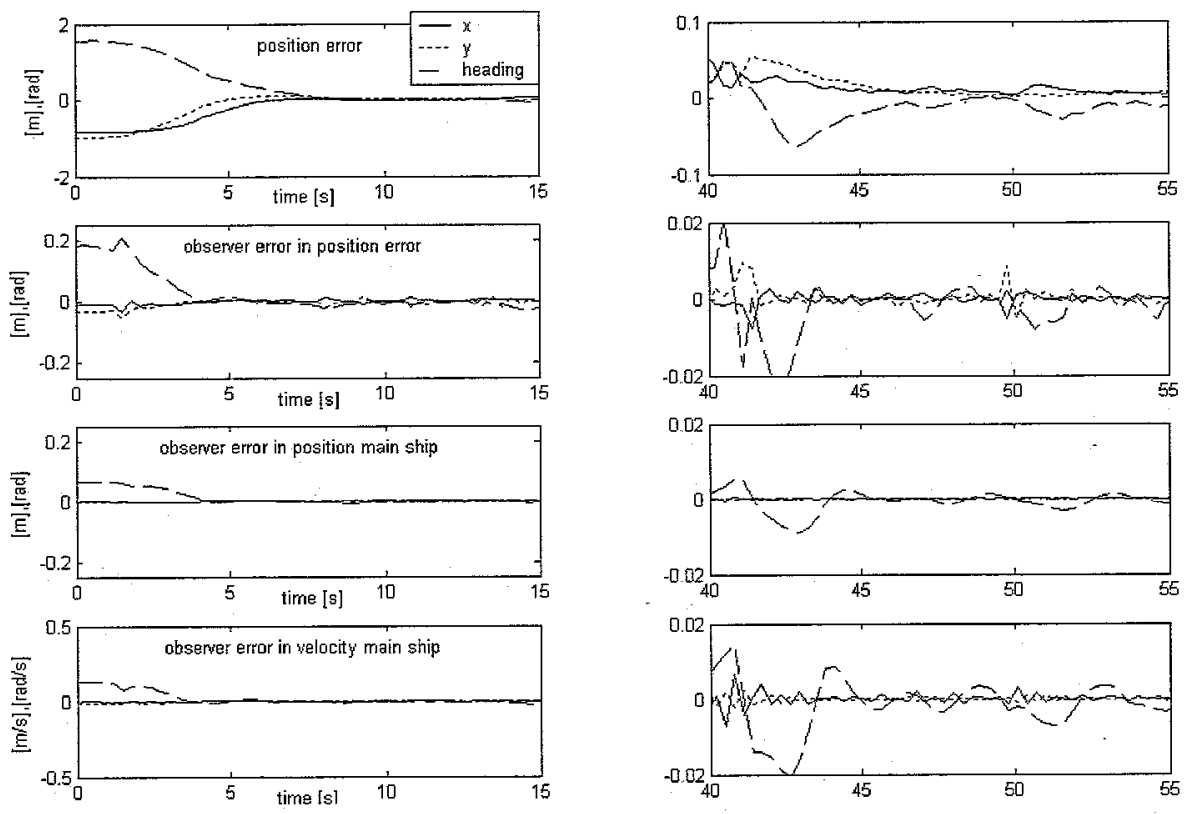

Figure I.1.1a Experimental result during position keeping.
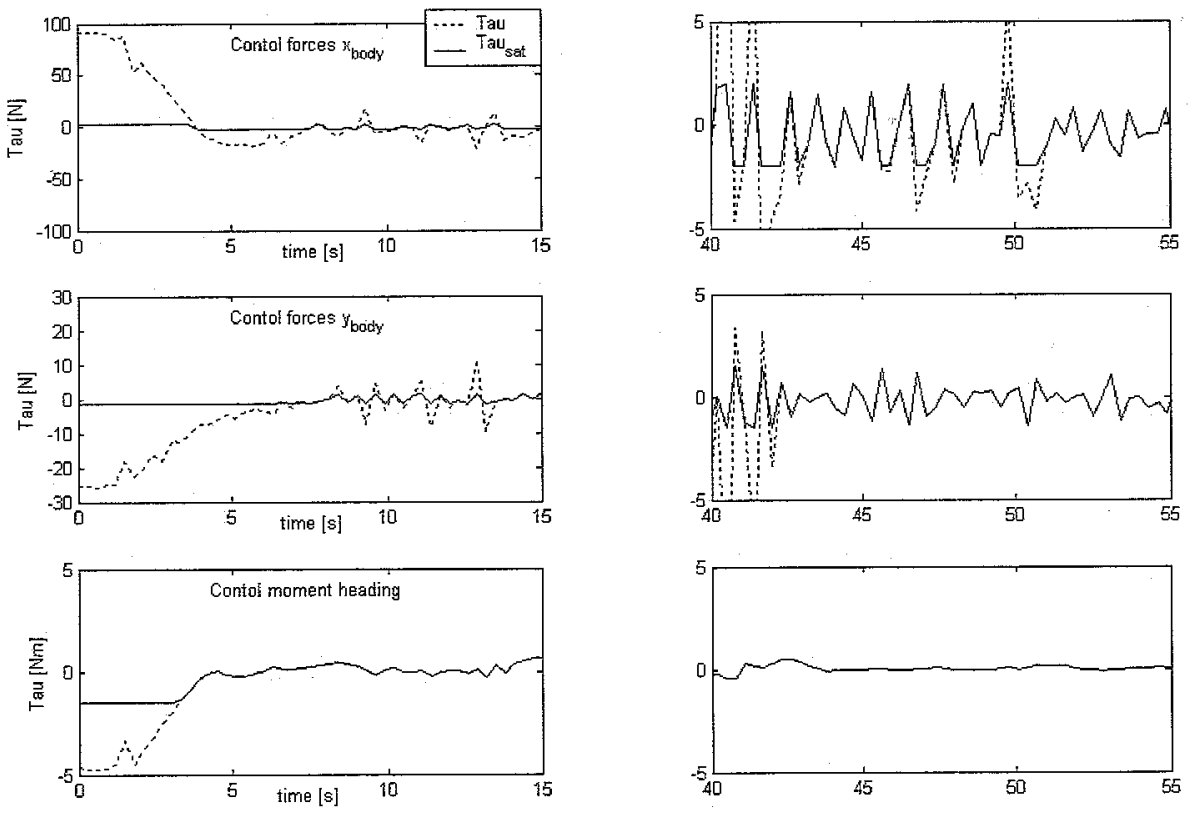

Figure I.1.1b Control forces calculated and saturated during position keeping. 
Simulation under ideal conditions position keeping gain set 1 :
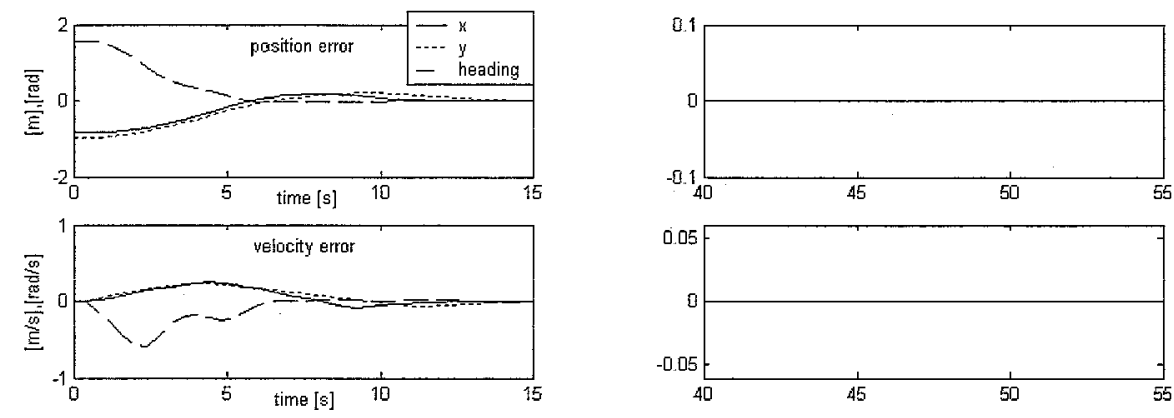

Figure I.1.2a Simulation results of the control errors during position keeping.
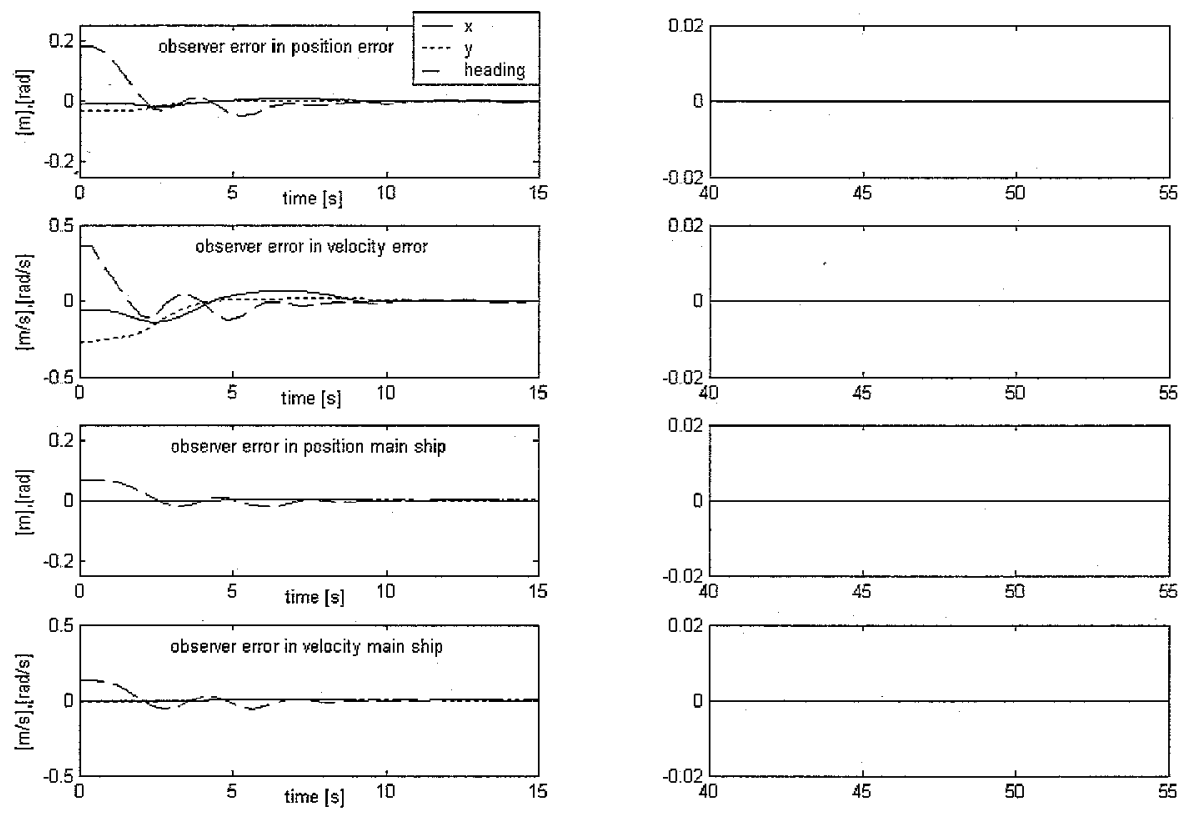

Figure I.1.2b Simulation results of the observer errors during position keeping.
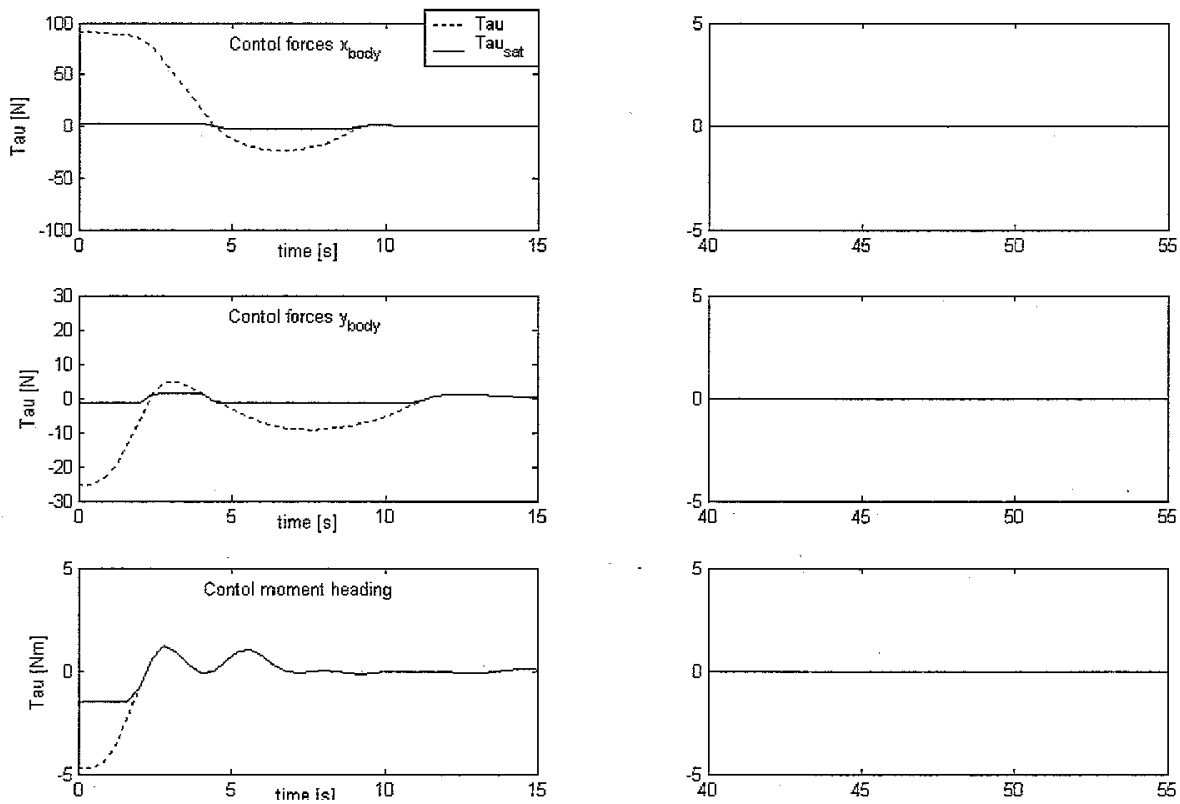

Figure I.1.2c Control forces calculated and saturated during position keeping. 
Simulation with non-linear damping position keeping gain set 1 :
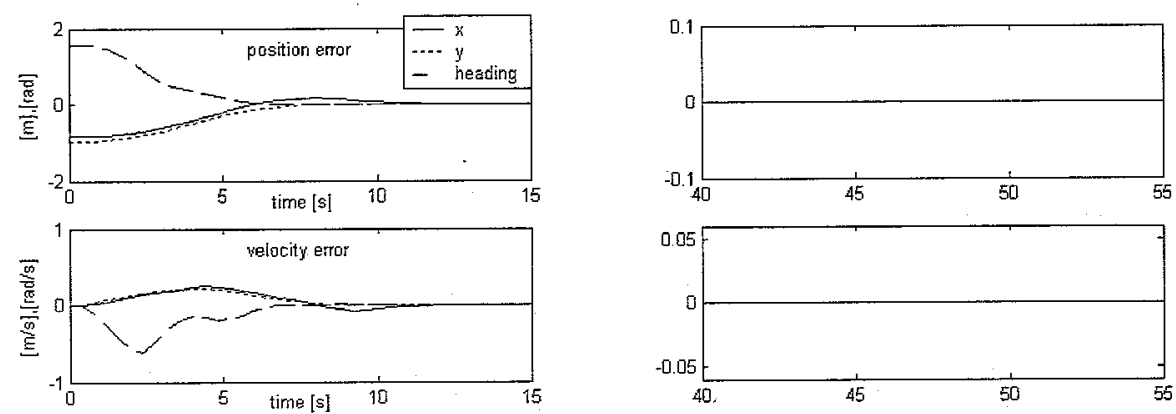

Figure I.1.3a Simulation results of the control errors during position keeping.
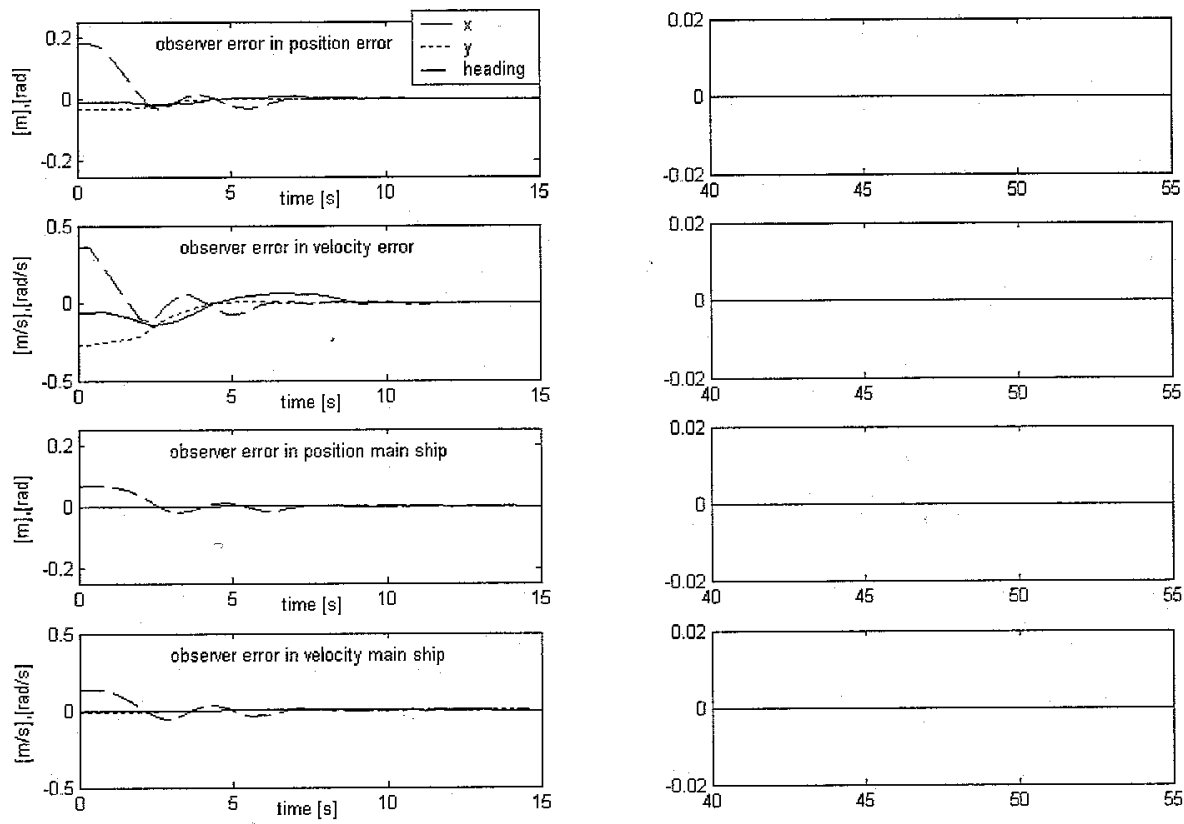

Fiqure I.1.3b Simulation results of the observer errors during position keeping.
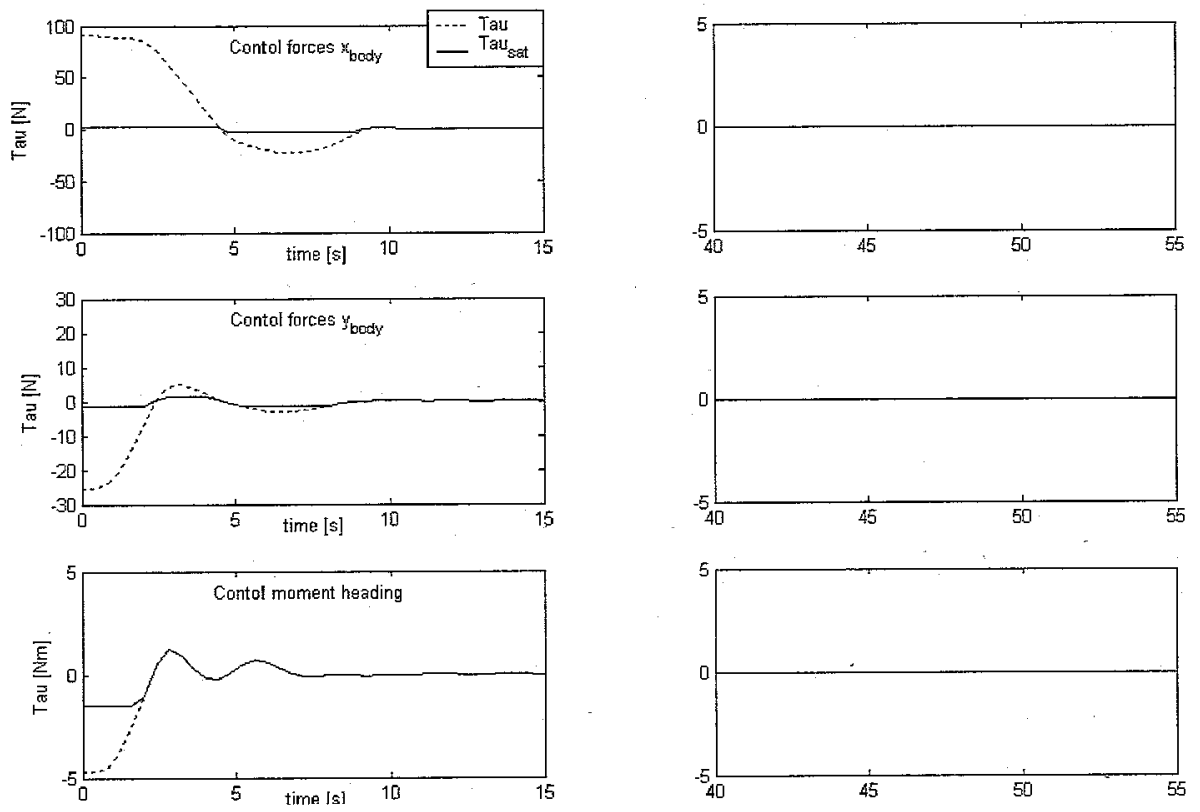

Figure I.1.3c Control forces calculated and saturated during position keeping. 
Simulation with non-linear damping and simulated measurement noise position keeping gain set 1 :
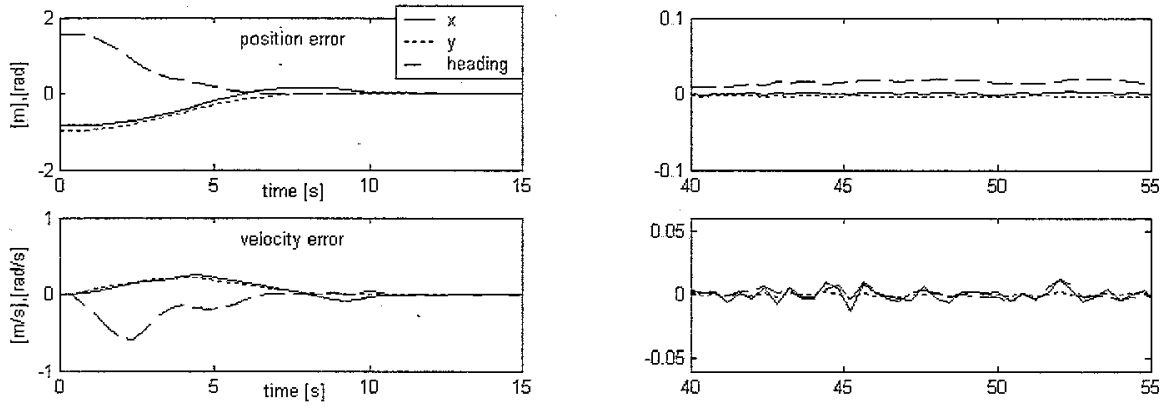

Figure I.1.4a Simulation results of the control errors during position keeping.
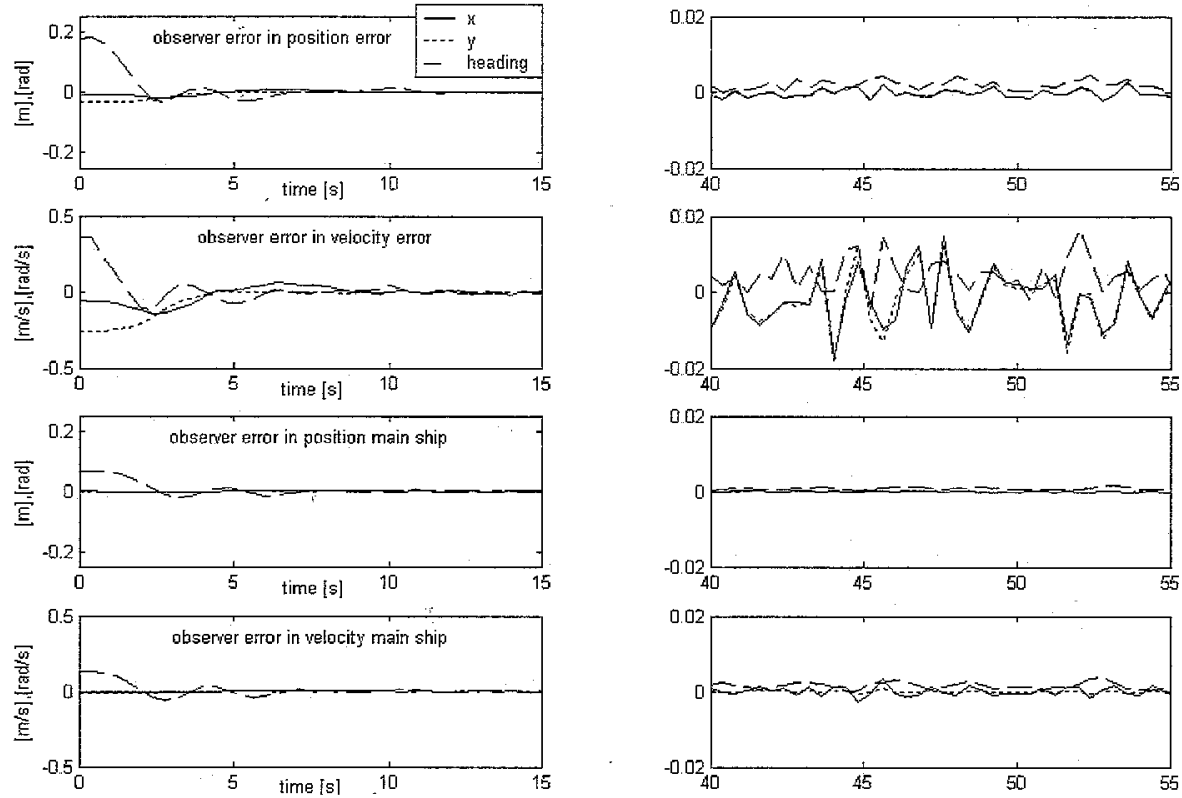

Figure I.1.4b Simulation results of the observer errors during position keeping.
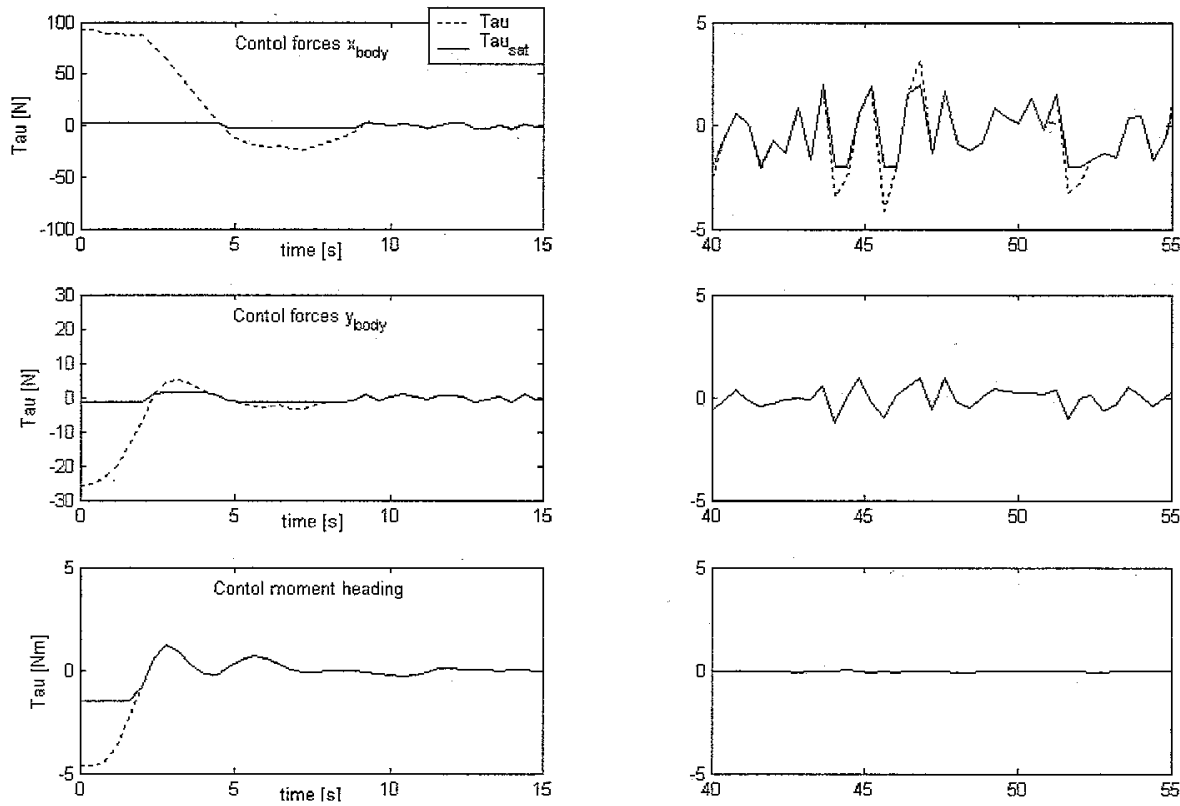

Figure I.1.4c Control forces calculated and saturated during position keeping. 
Experiment position keeping gain set 2:
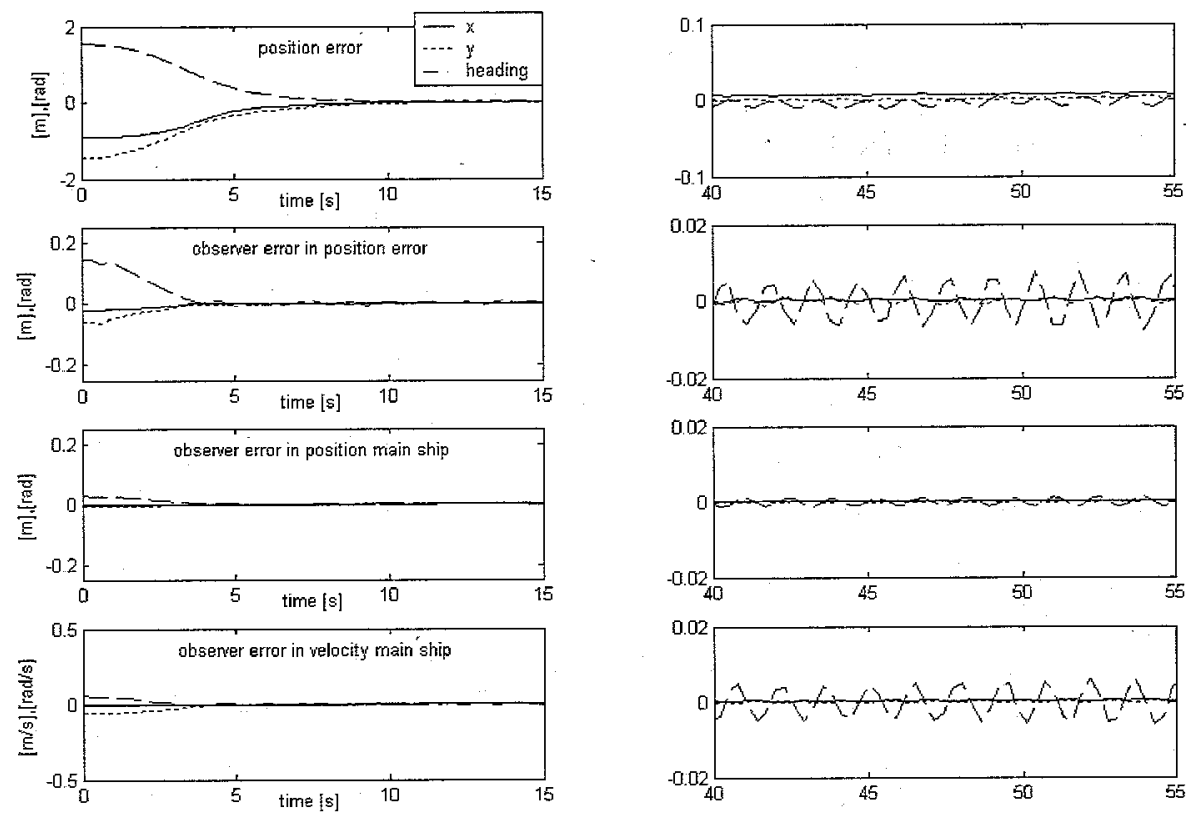

Figure 1.2.1a experimental result position keeping.
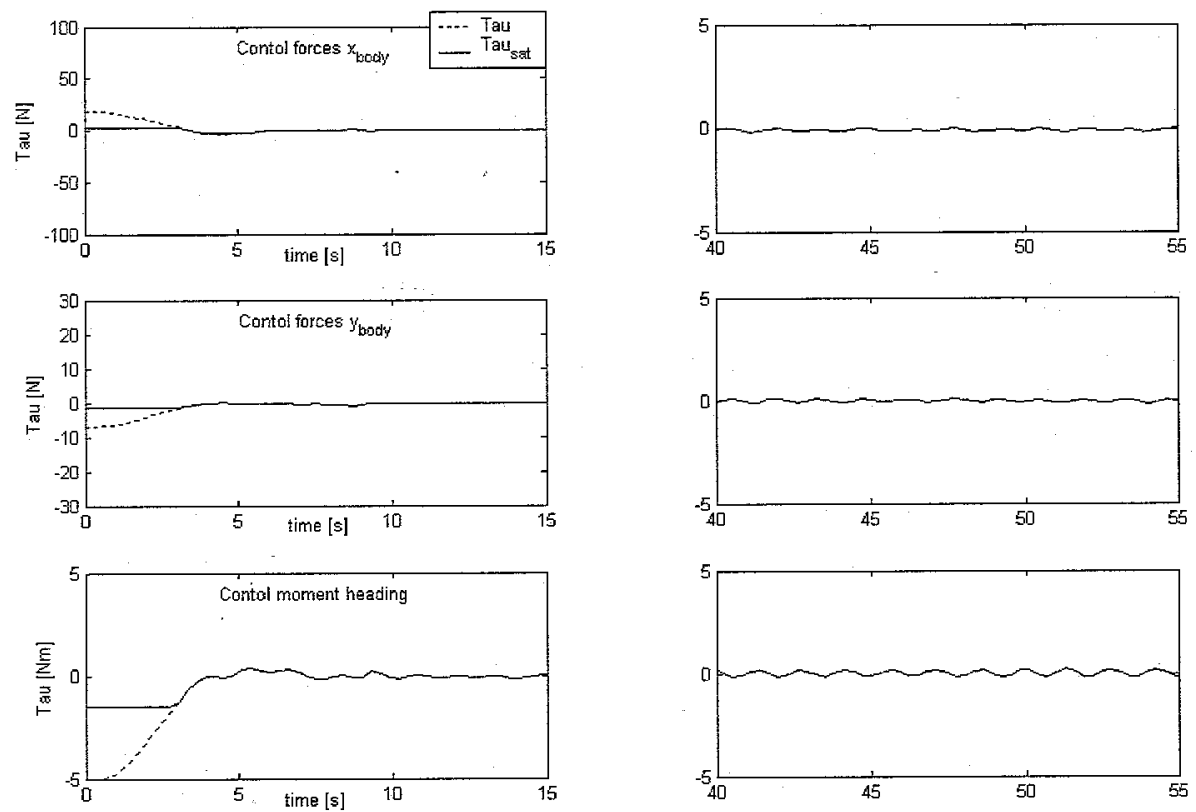

Figure I.2.1b Control forces calculated and saturated during position keeping. 
Simulation under ideal conditions position keeping gain set 2:
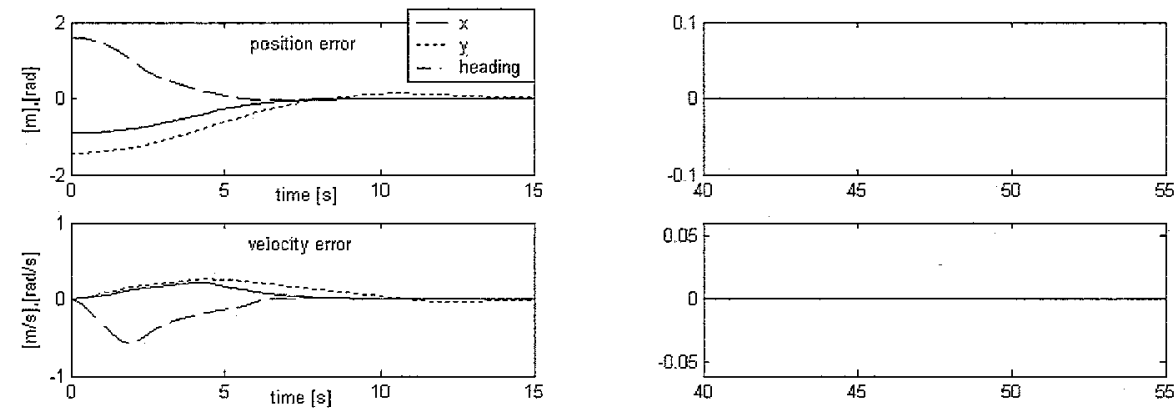

Figure 1.2.2a Simulation results of the control errors during position keeping.
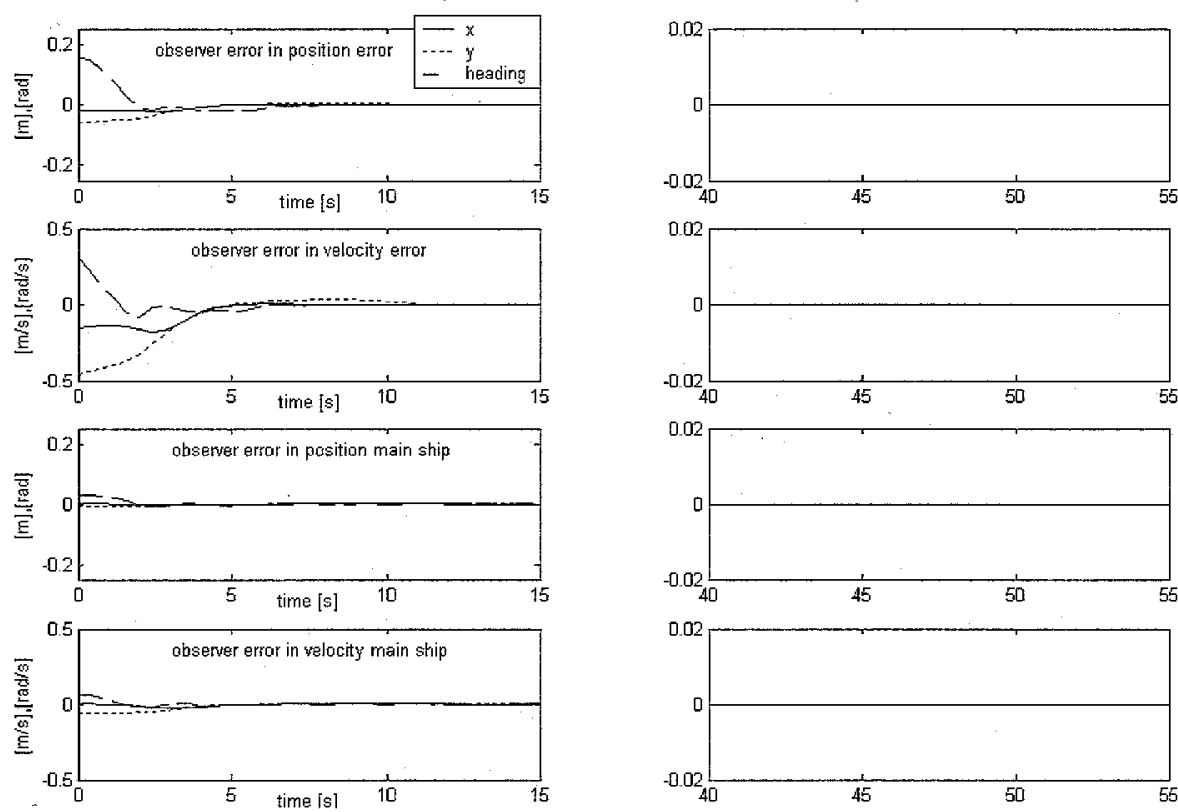

Figure I.2.2b Simulation results of the observer errors during position keeping.
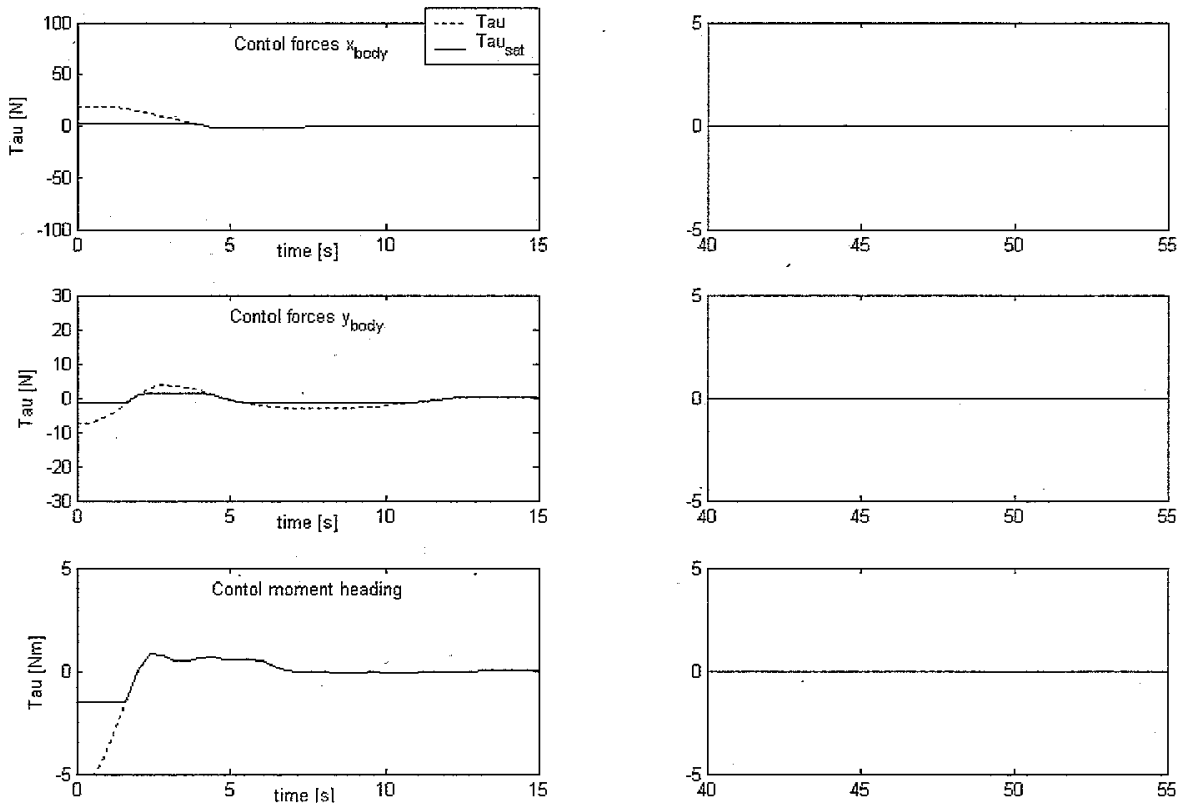

Figure I.2.2c Control forces calculated and saturated during position keeping. 
Simulation with non-linear damping position keeping gain set 2:
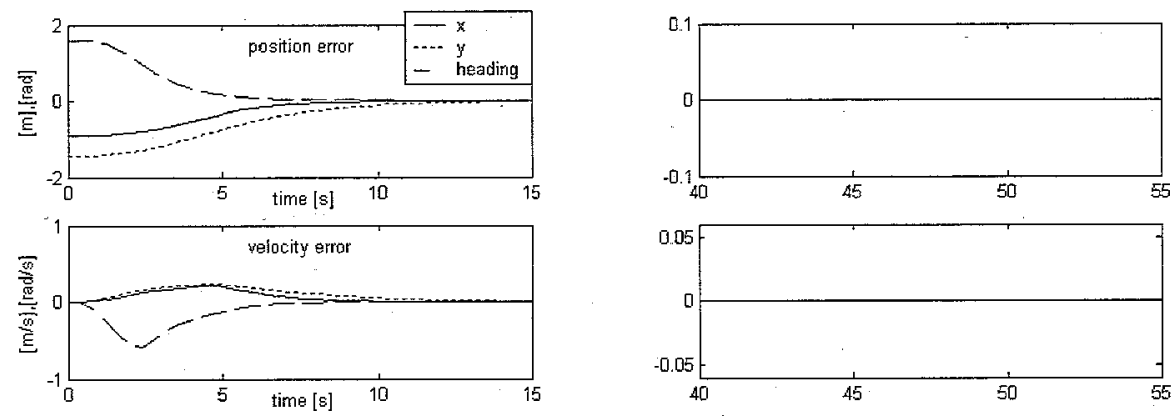

Figure 1.2.3a Simulation results of the control errors during position keeping.
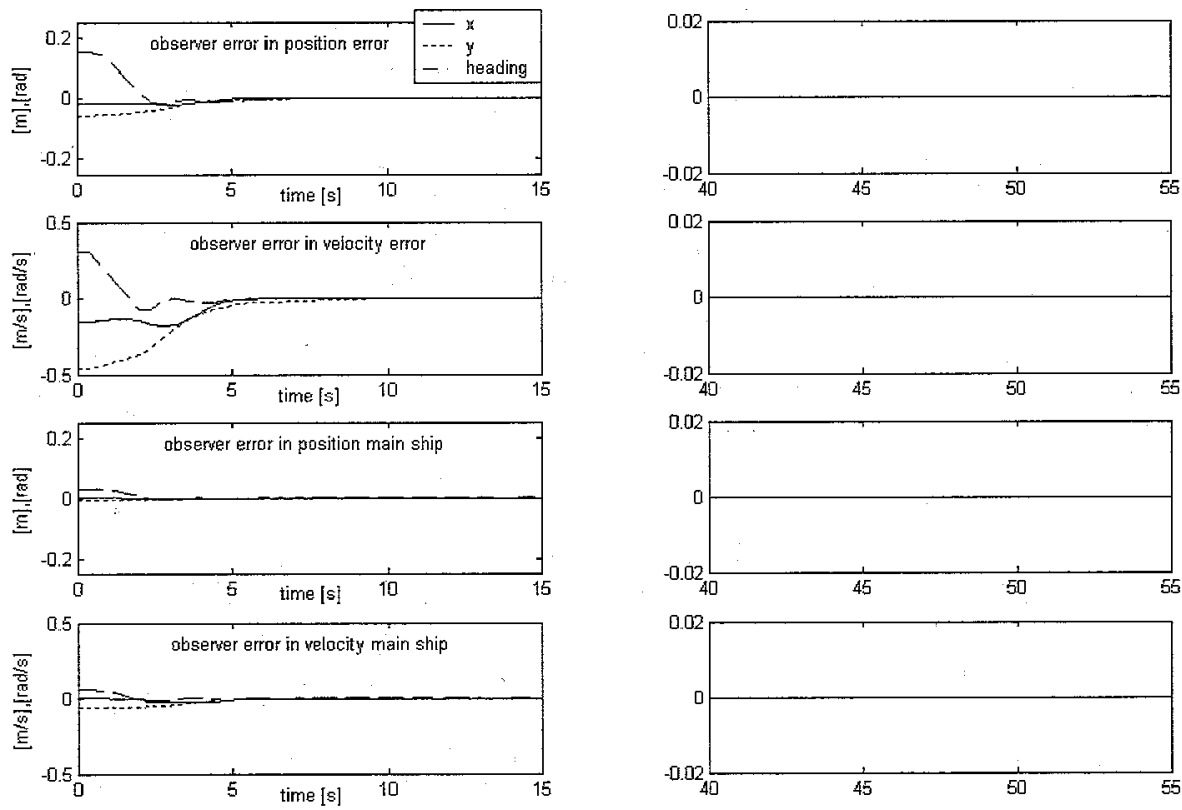

Figure I.2.3b Simulation results of the observer errors during position keeping.
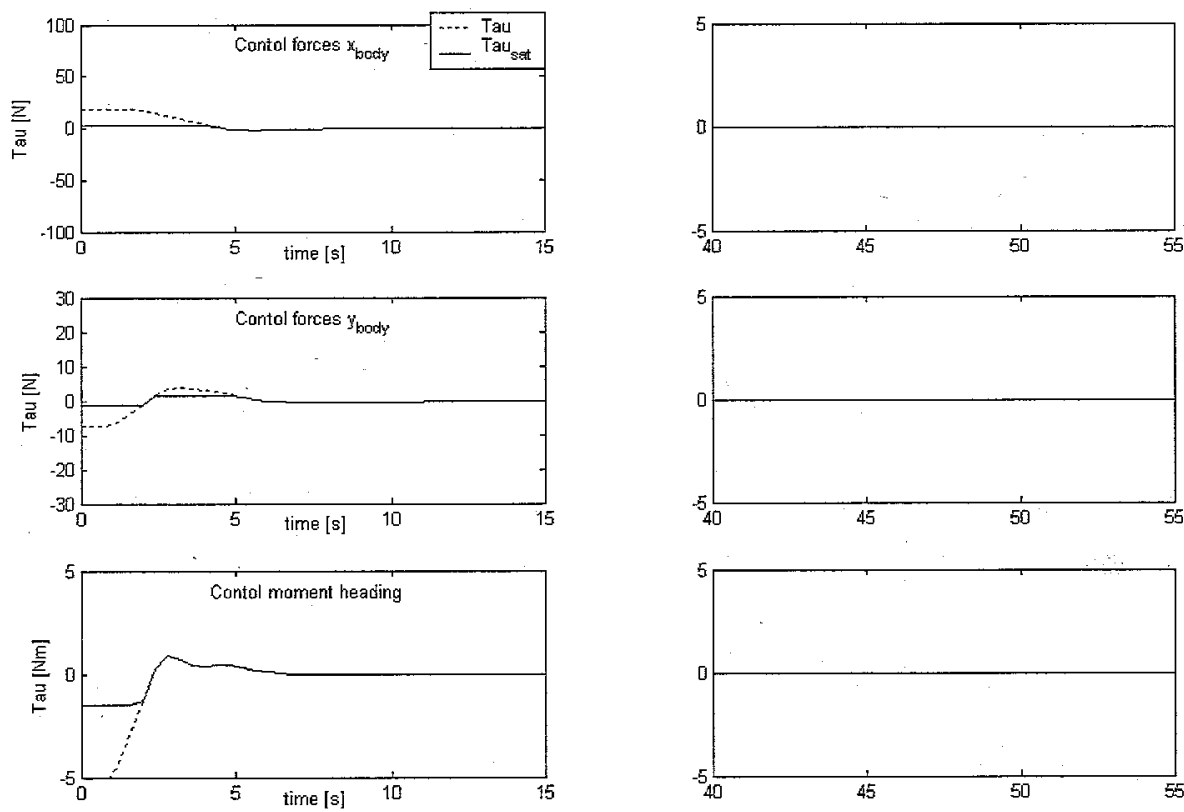

Figure I.2.3c Control forces calculated and saturated during position keeping. 
Simulation with non-linear damping and simulated measurement noise position keeping gain set 2:
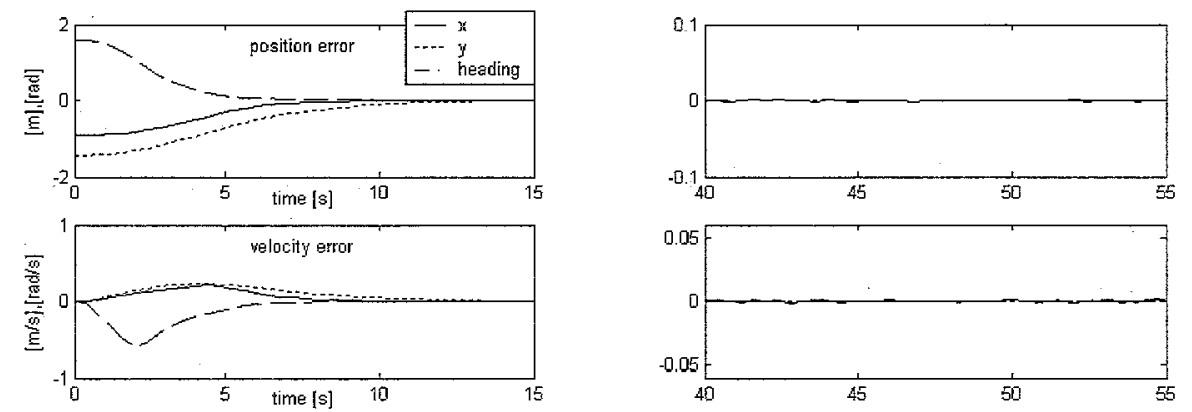

Figure I.2.4a Simulation results of the control errors during position keeping.
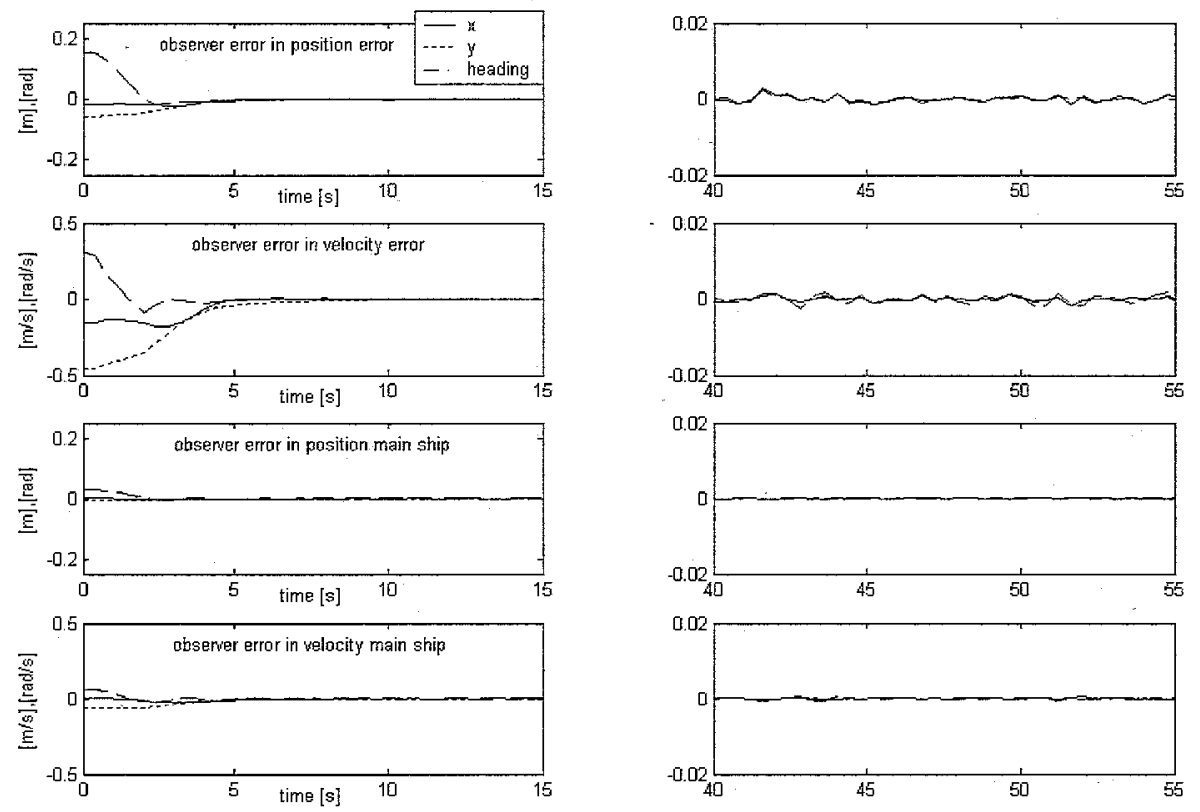

Figure I.2.4b Simulation results of the observer errors during position keeping.
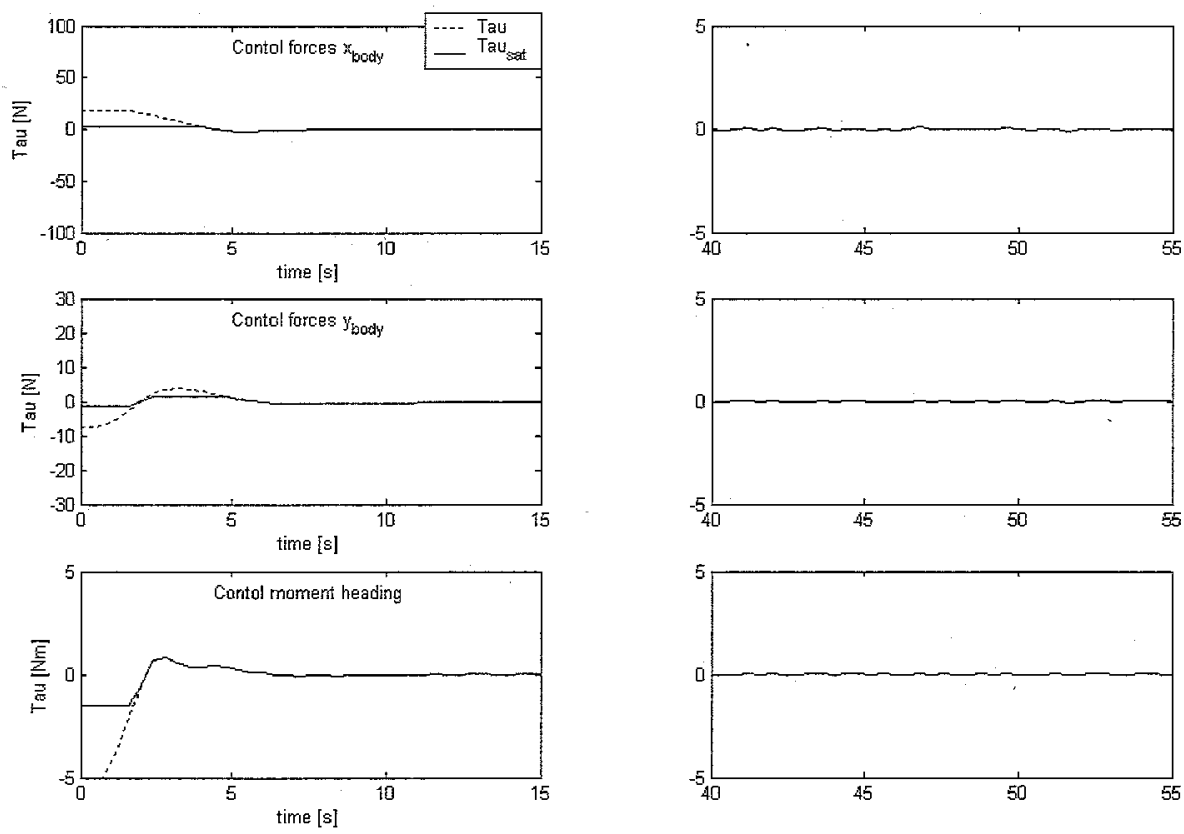

Figure I.2.4c Control forces calculated and saturated during position keeping. 


\section{Appendix I: Figures trajectory tracking gain set 1 and set 3.}

Experiment trajectory tracking gain set 1 :
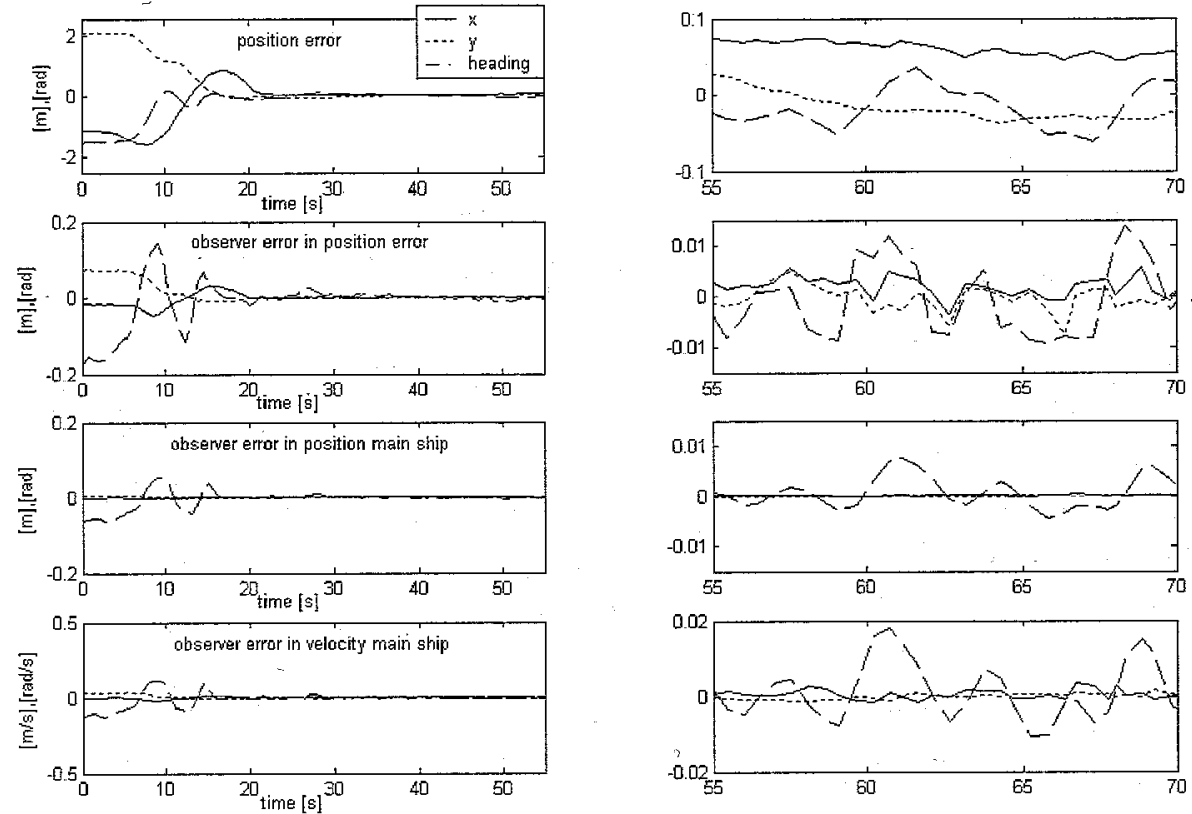

Figure J.1.1a experimental result during trajectory tracking.
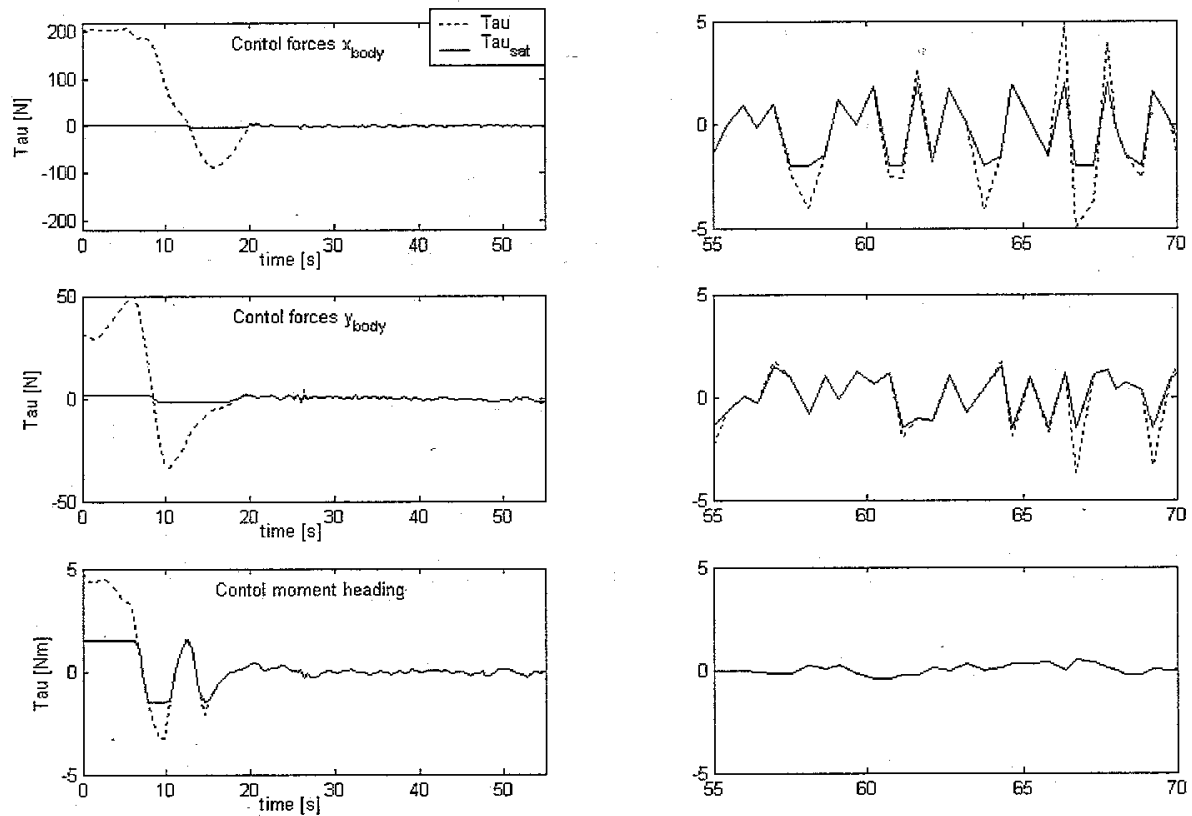

Figure J.1.1b Control forces calculated and saturated during trajectory tracking. 
Simulation under ideal conditions trajectory tracking gain set 1 :
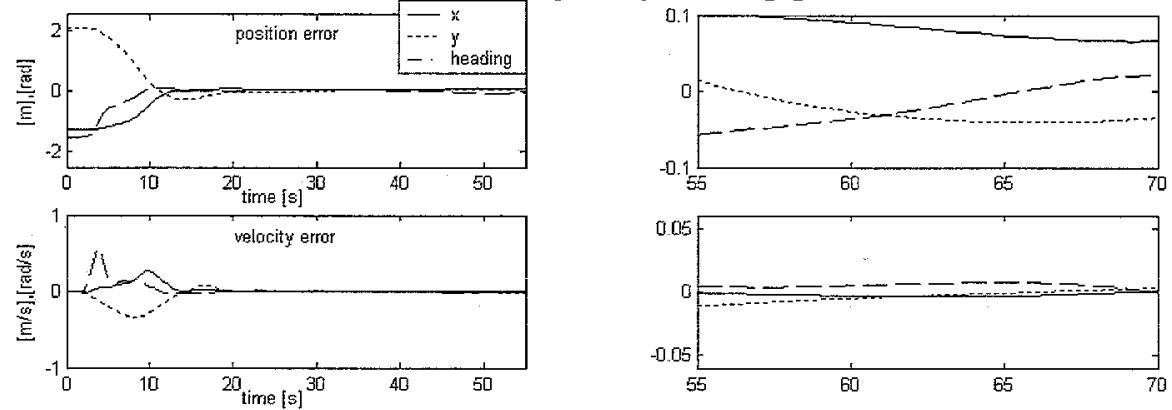

Figure J.1.2a Simulation results of the control errors during trajectory tracking.
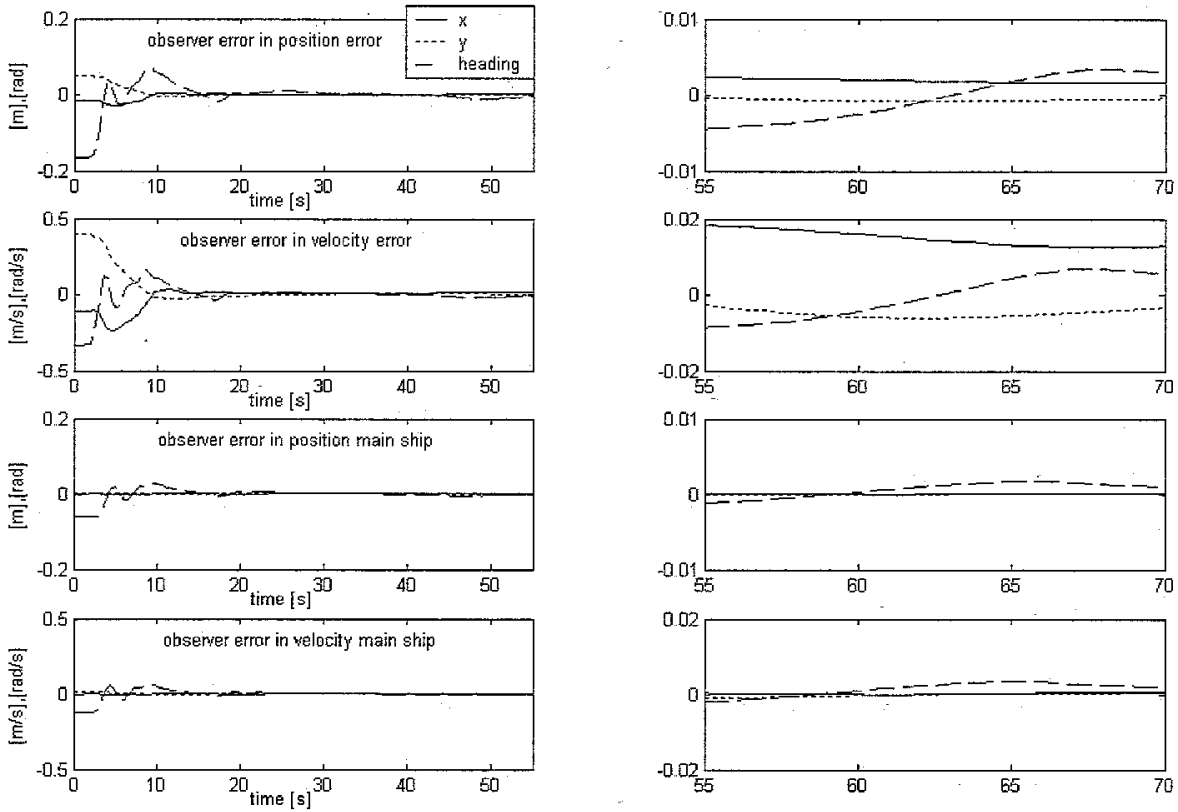

Figure J.1.2b Simulation results of the observer errors during trajectory tracking.
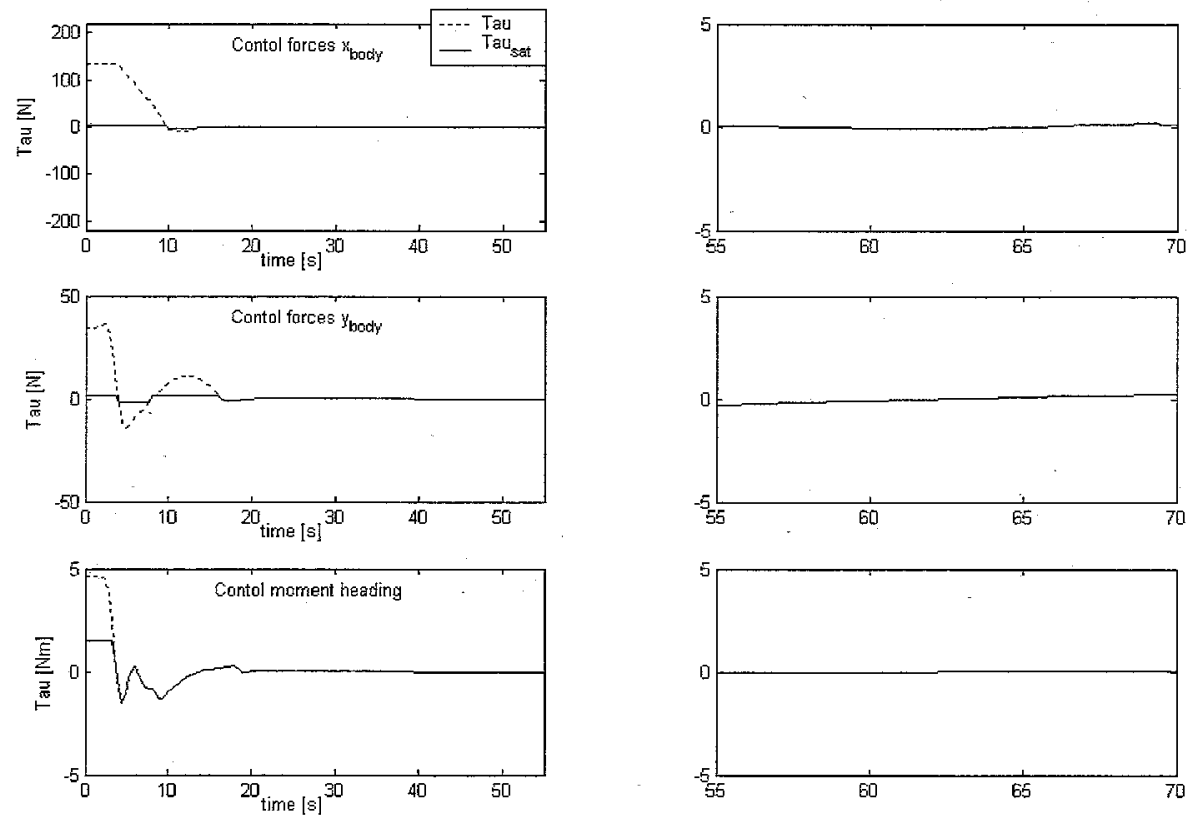

Figure J.1.2c Control forces calculated and saturated during trajectory tracking. 
Simulation with non-linear damping trajectory tracking gain set 1 :
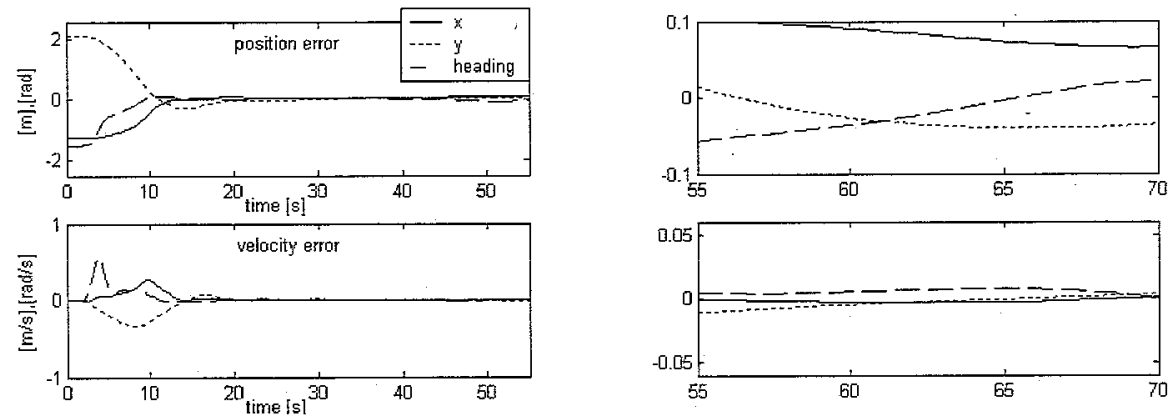

Figure J.1.3a Simulation results of the control errors during trajectory tracking.
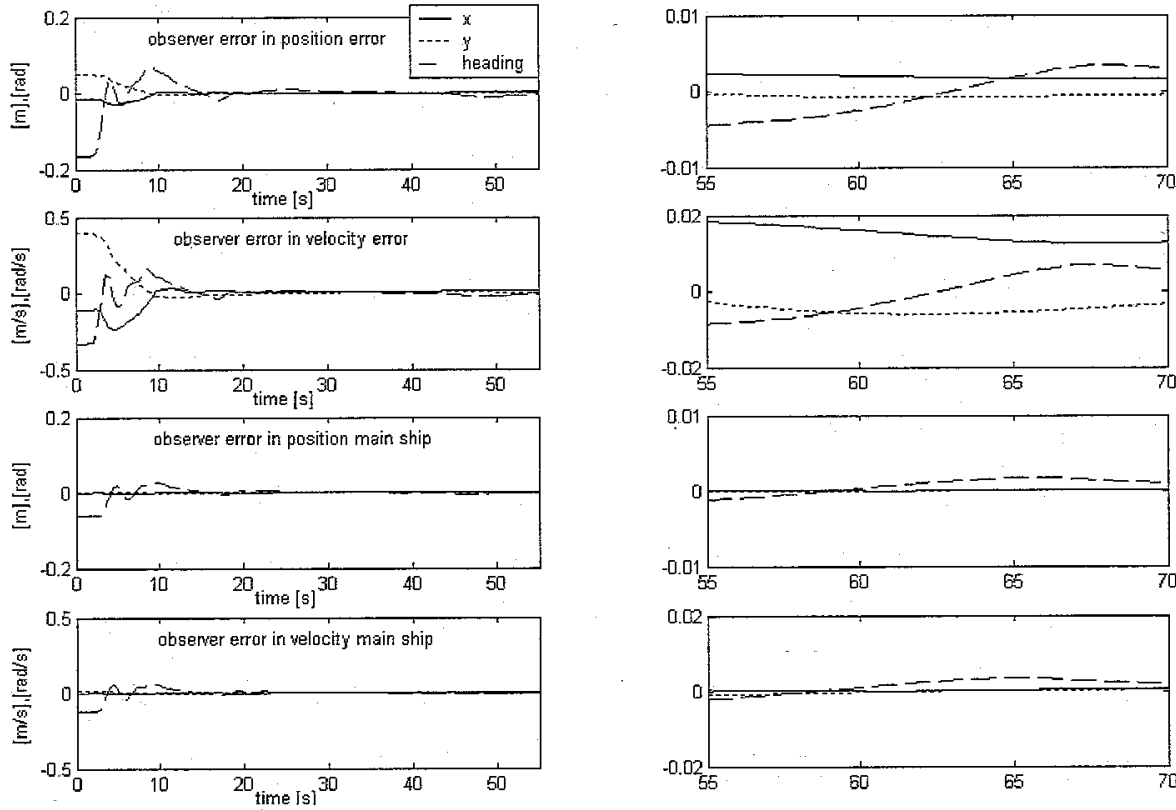

Figure J.1.3b Simulation results of the observer errors during trajectory tracking.
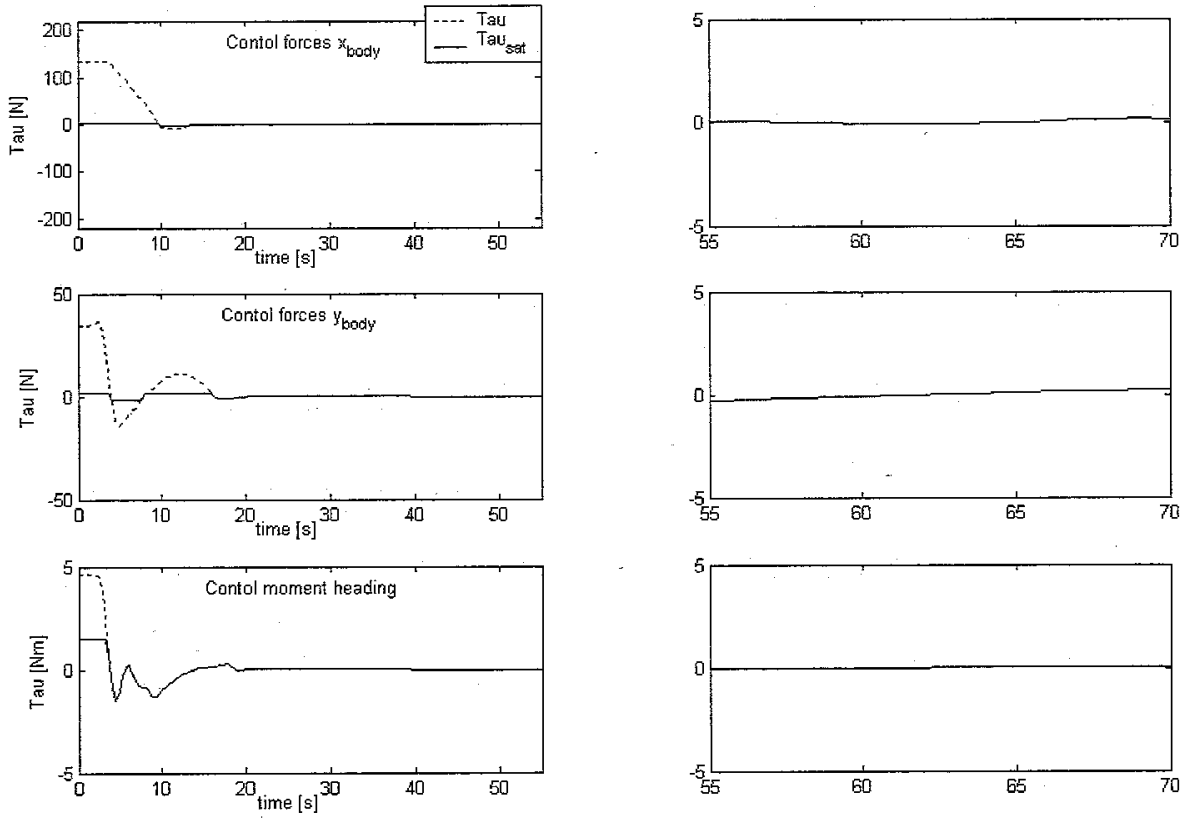

Figure J.1.3c Control forces calculated and saturated during trajectory tracking. 
Simulation with non-linear damping and simulated measurement noise trajectory tracking gain set 1 :
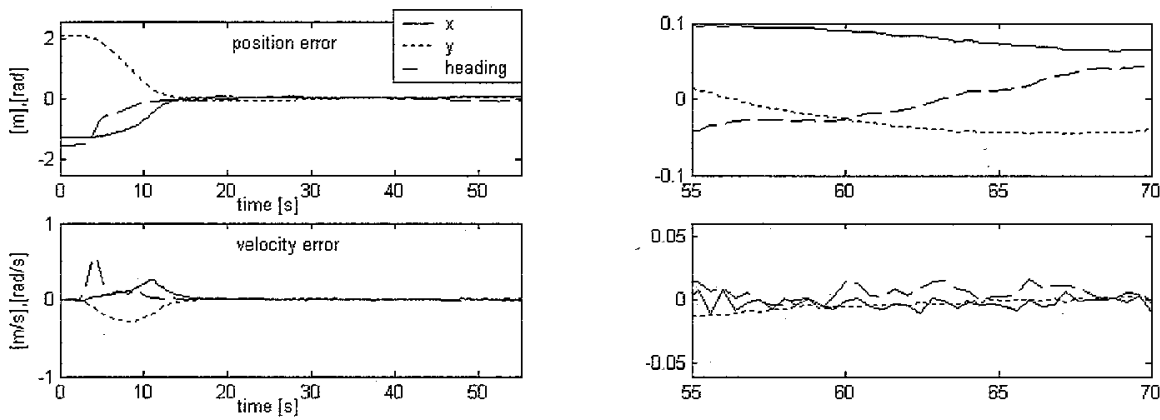

Figure J.1.4a Simulation results of the control errors during trajectory tracking.
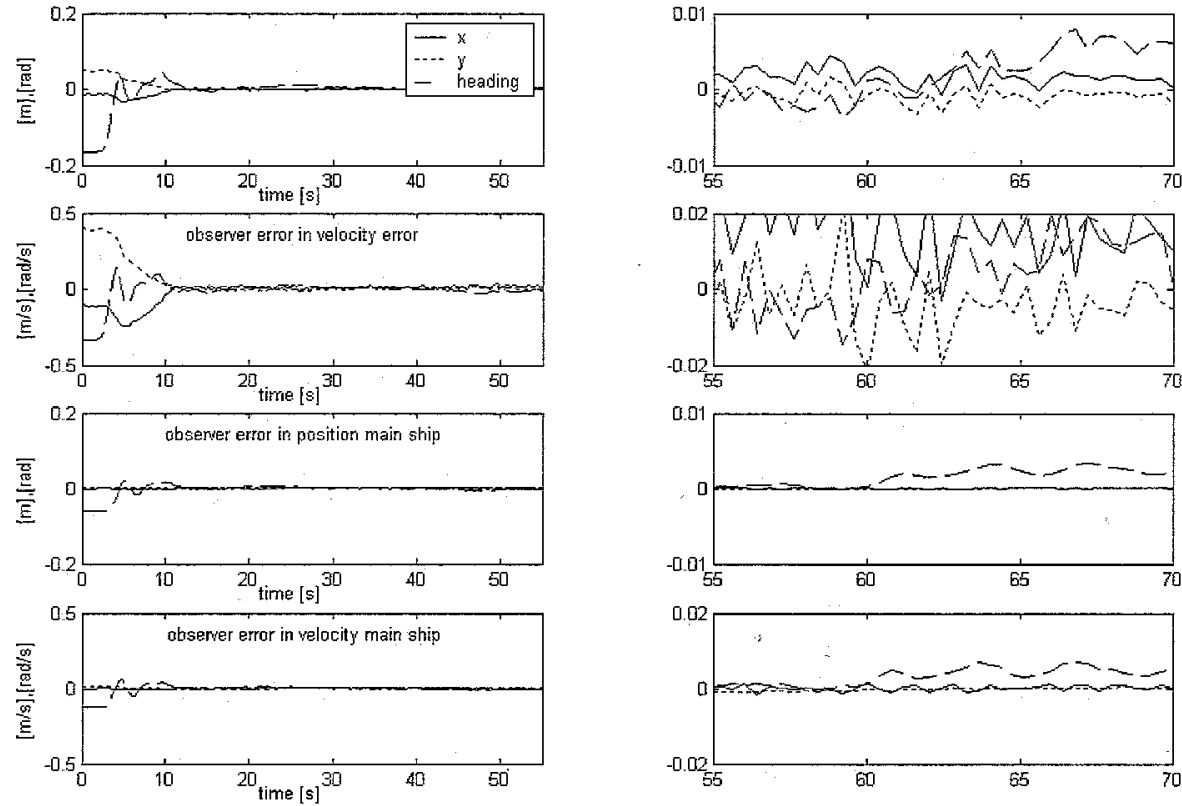

Figure J.1.4b Simulation results of the observer errors during trajectory tracking.
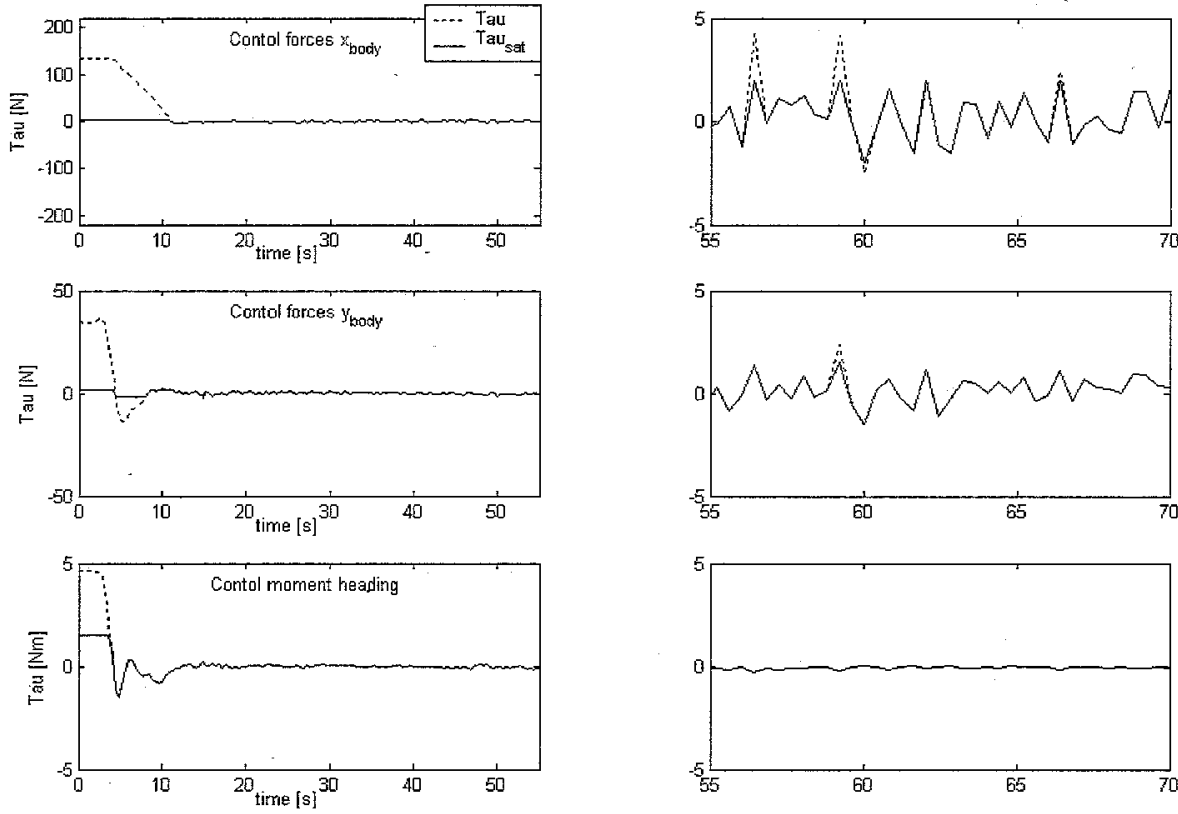

Figure J.1.4c Control forces calculated and saturated during trajectory tracking. 
Experiment trajectory tracking gain set 3:
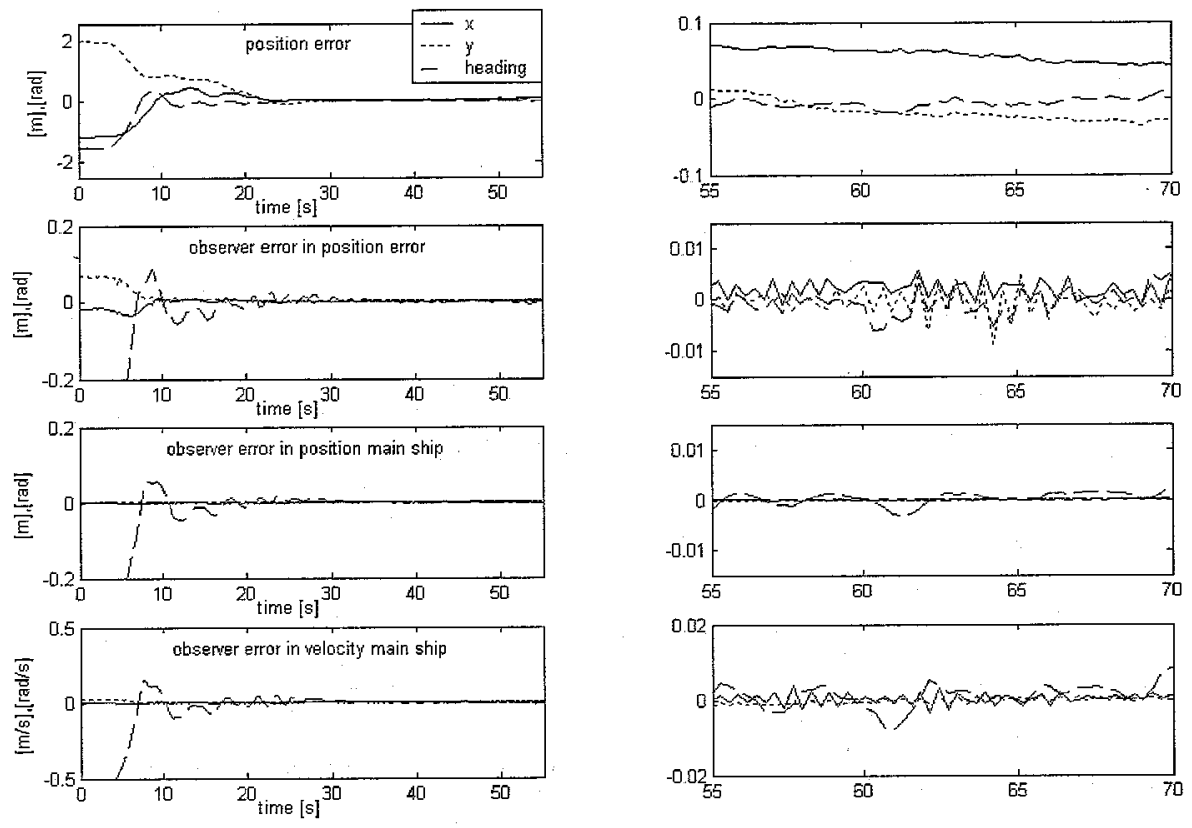

Figure 3.2.1a experimental result during trajectory tracking.
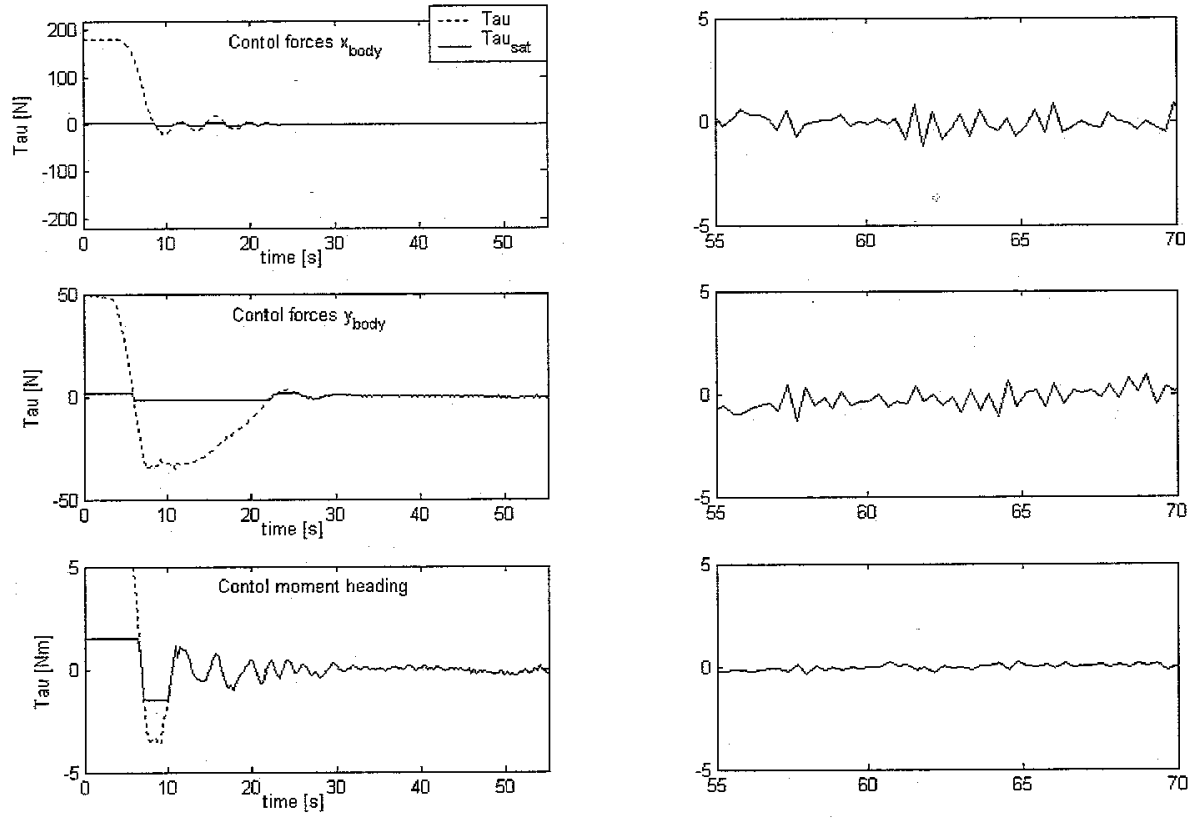

Figure J.2.1b Control forces calculated and saturated during trajectory tracking. 
Simulation under ideal conditions trajectory tracking gain set 3 :
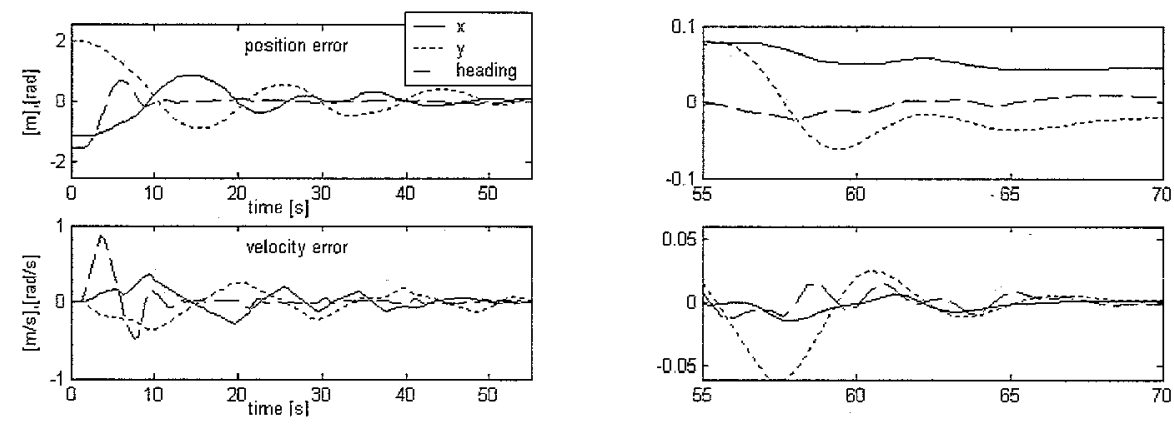

Figure 3.2.2a Simulation results of the control errors during trajectory tracking.
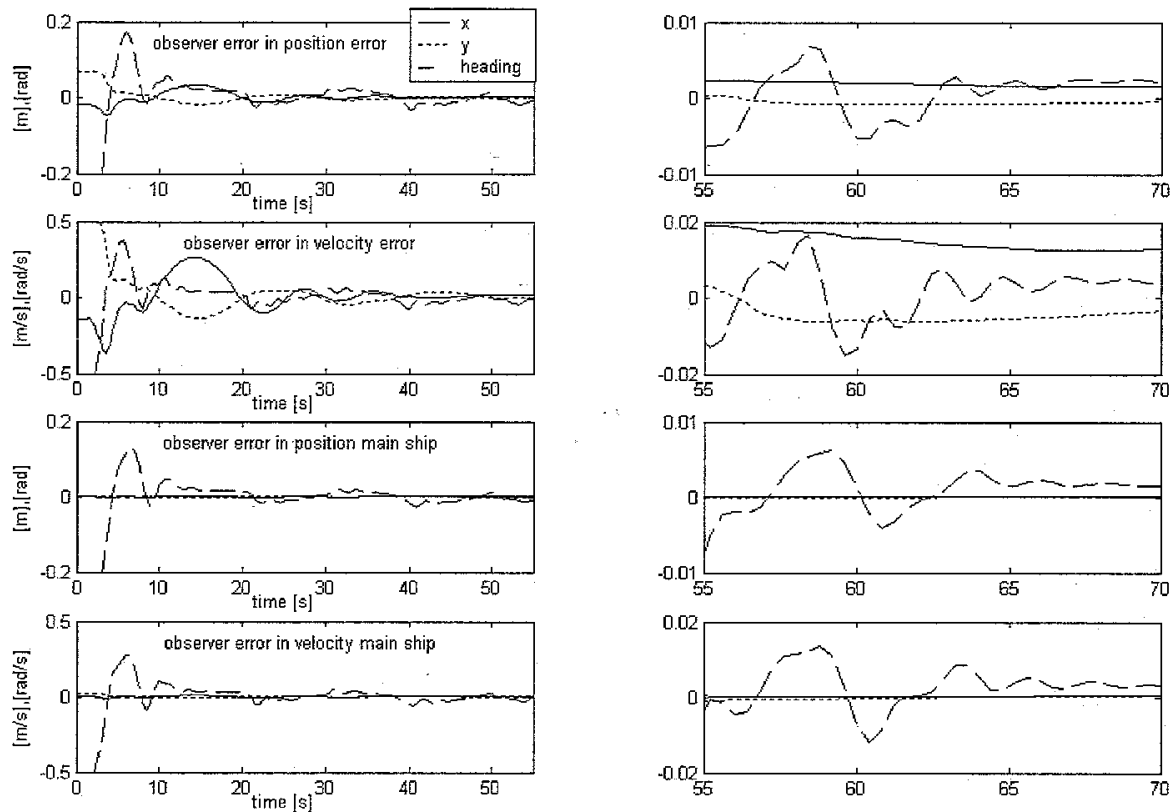

Figure J.2.2b Simulation results of the observer errors during trajectory tracking.
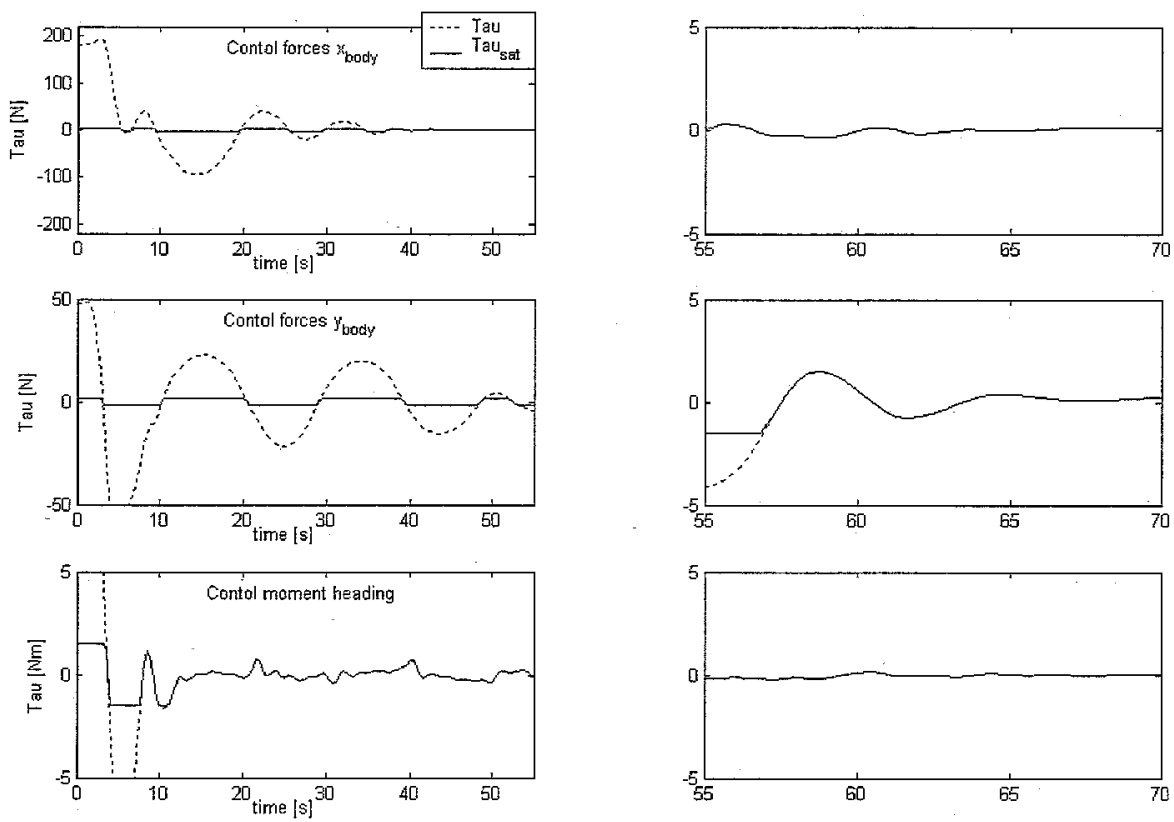

Figure J.2.2c Control forces calculated and saturated during trajectory tracking. 
Simulation with non-linear damping trajectory tracking gain set 3 :
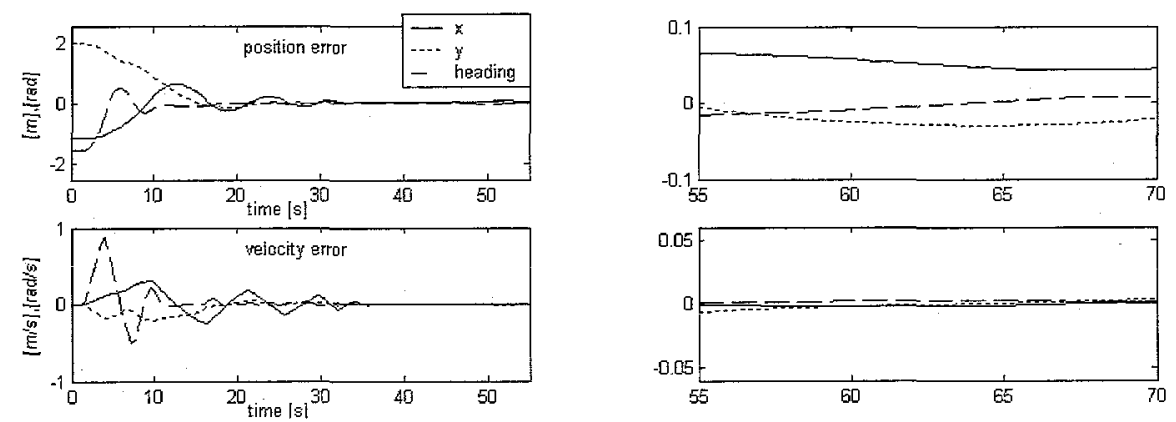

Figure 3.2.3a Simulation results of the control errors during trajectory tracking.
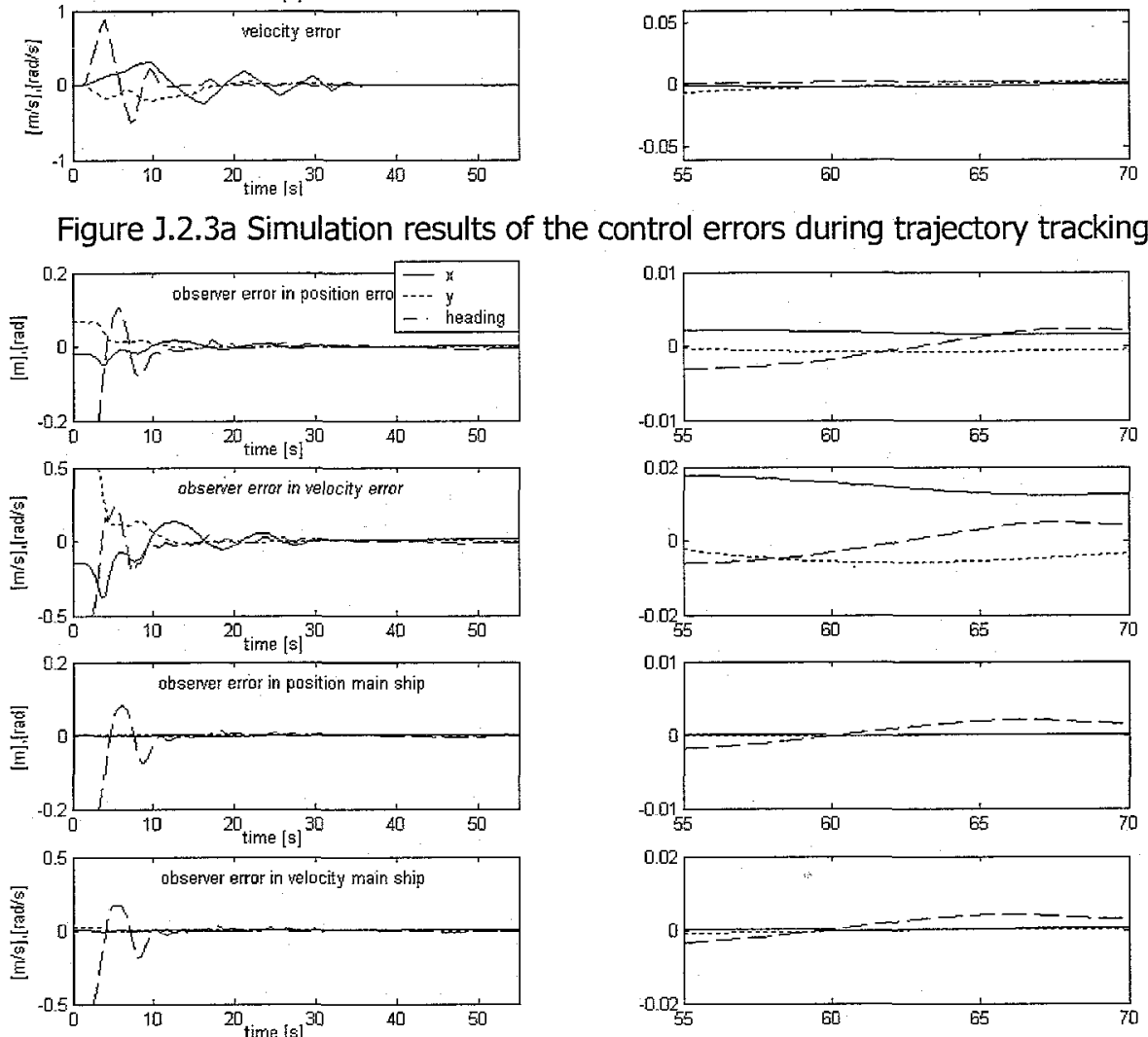

Figure J.2.3b Simulation results of the observer errors during trajectory tracking.
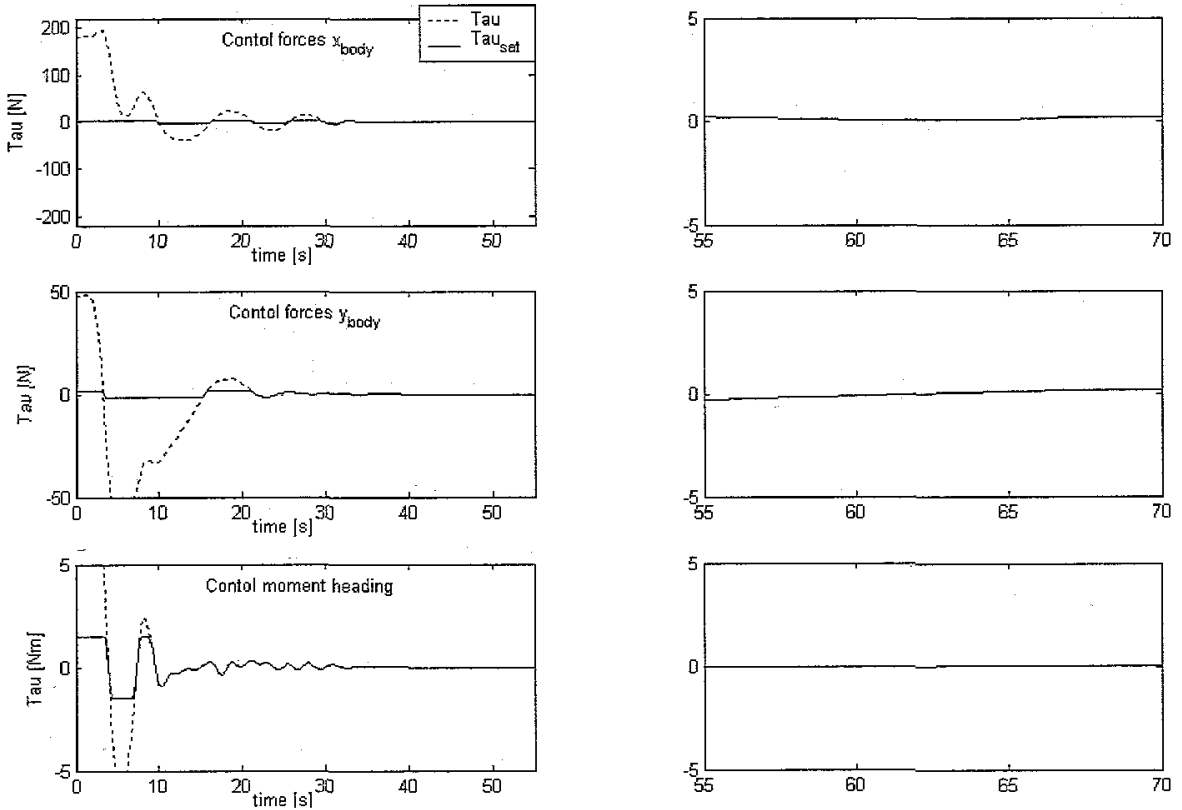

Figure J.2.3c Control forces calculated and saturated during trajectory tracking. 
simulation with non-linear damping and simulated measurement noise trajectory tracking gain set 3 :
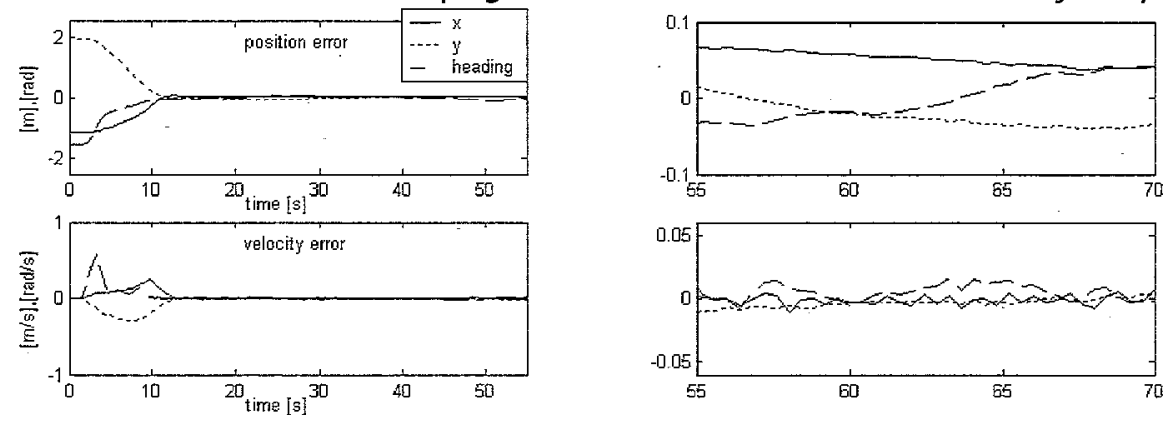

Figure 3.2.4a Simulation results of the control errors during trajectory tracking.
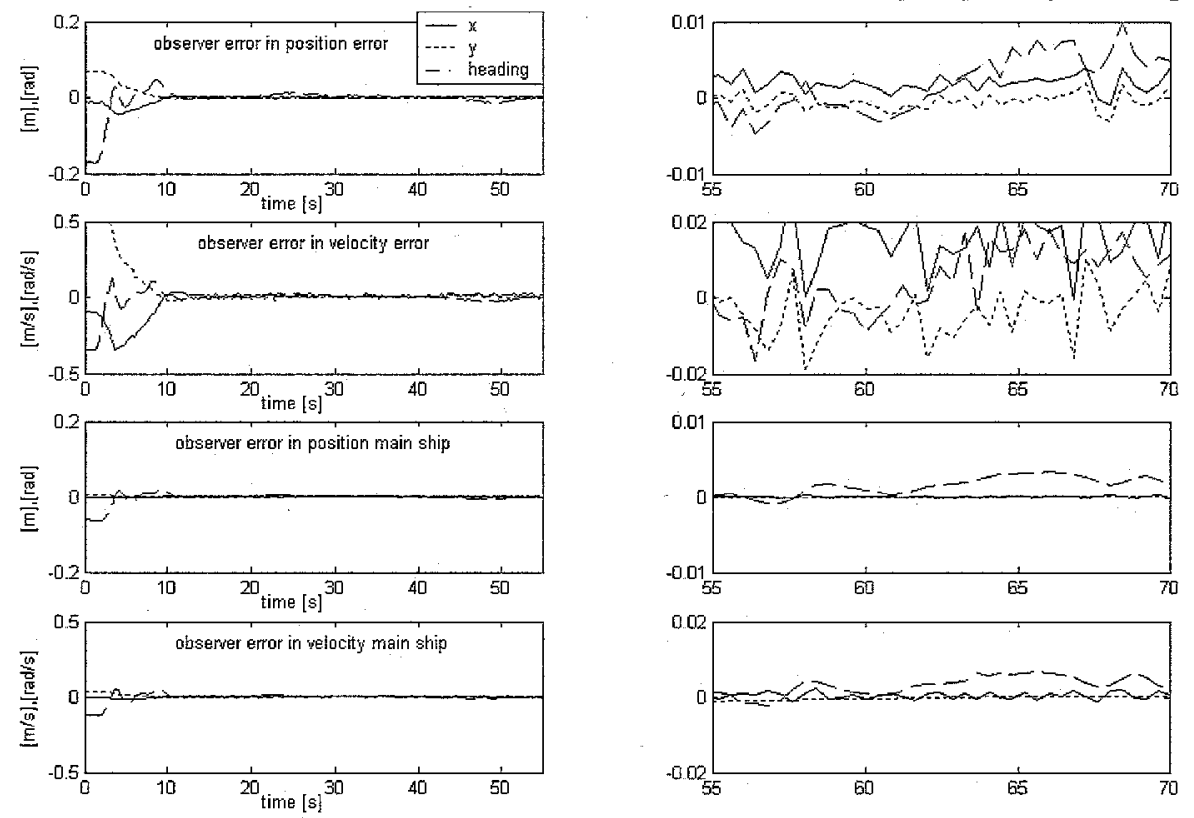

Figure J.2.4b Simulation results of the observer errors during trajectory tracking.
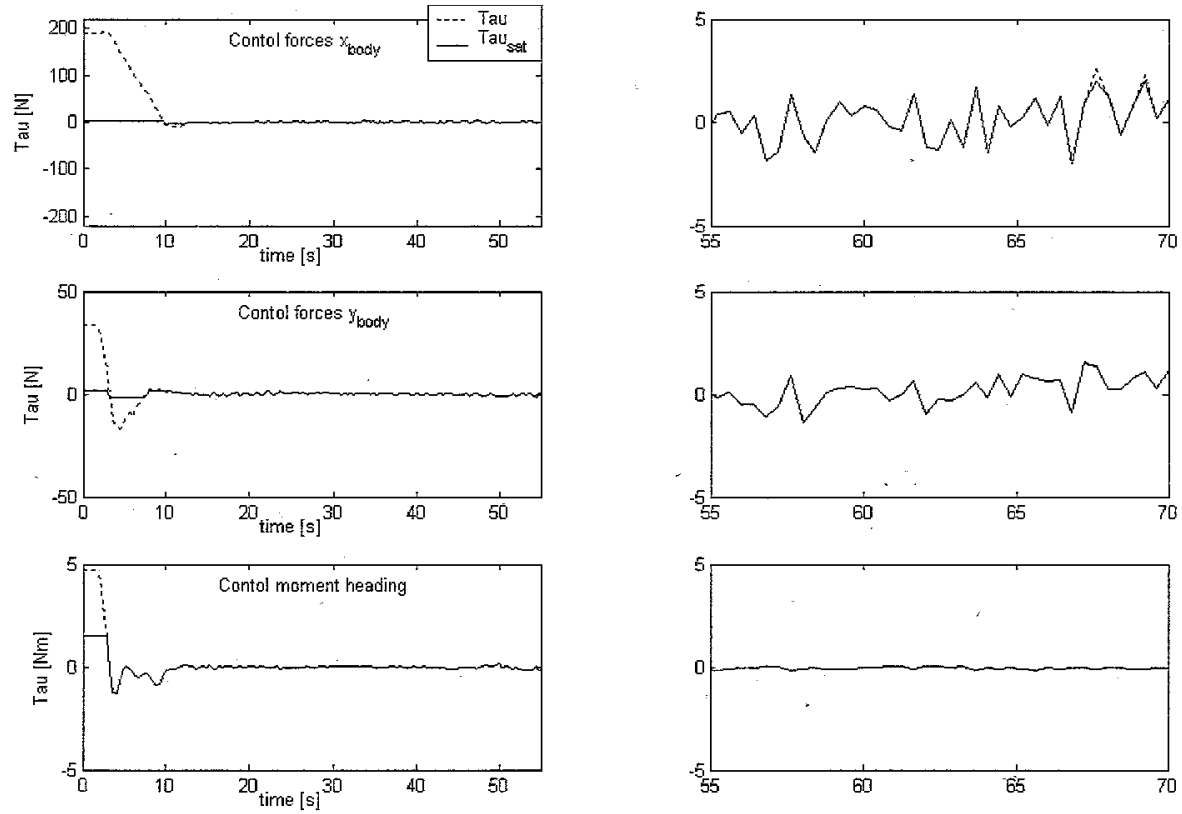

Figure J.2.4c Control forces calculated and saturated during trajectory tracking. 


\section{Appendix J: Table performance tracking straight line}

\begin{tabular}{|c|c|c|c|c|c|c|}
\hline \multicolumn{7}{|c|}{ Experiments: line } \\
\hline \multirow[b]{3}{*}{ ud $[\mathrm{m} / \mathrm{s}]$} & \multicolumn{6}{|c|}{ Without waves } \\
\hline & \multicolumn{2}{|c|}{ x_body $[\mathrm{m}]$} & \multicolumn{2}{|c|}{ y_body $[\mathrm{m}]$} & \multicolumn{2}{|c|}{ heading [deg] } \\
\hline & mean IeI & $\max$ IeI & mean IeI & $\max$ IeI & mean IeI & $\max$ IeI \\
\hline 0.1 & 0.0317 & 0.0417 & 0.0040 & 0.0129 & 0.6589 & 2.7731 \\
\hline 0.2 & 0.0584 & 0.0709 & 0.0021 & 0.0080 & 0.4469 & 2.0626 \\
\hline 0.3 & 0.0847 & 0.0931 & 0.0021 & 0.0072 & 1.1975 & 2.4752 \\
\hline \multirow[t]{2}{*}{0.4} & 0.1077 & 0.1189 & 0.0021 & 0.0062 & 2.9565 & 3.8617 \\
\hline & \multicolumn{6}{|c|}{ With waves ( Johnswap: $\mathrm{Hs}=0.01 \mathrm{~m}, \mathrm{Ts}=0.75 \mathrm{~s}$ ) } \\
\hline 0.1 & 0.0379 & 0.0652 & 0.0044 & 0.0116 & 1.1459 & 4.1024 \\
\hline 0.2 & 0.0620 & 0.0814 & 0.0034 & 0.0092 & 0.9339 & 4.0737 \\
\hline 0.3 & 0.0868 & 0.1090 & 0.0042 & 0.0107 & 0.9969 & 2.8190 \\
\hline 0.4 & 0.1086 & 0.1162 & 0.0060 & 0.0131 & 2.1142 & 3.3805 \\
\hline
\end{tabular}

Table K.1a Experimental results during tracking straight line: gain set 1 .

\begin{tabular}{|c|cc|cc|cc|}
\hline \multicolumn{7}{|c|}{ Experiments: line } \\
\hline & \multicolumn{5}{|c|}{ Without waves } \\
\cline { 2 - 7 } & \multicolumn{2}{|c|}{ x_body [m] } & \multicolumn{2}{c|}{ y_body [m] } & \multicolumn{2}{c|}{ heading [deg] } \\
Ud [m/s] & mean IeI & max IeI & mean IeI & max IeI & mean IeI & max IeI \\
\hline 0.1 & 0.0283 & 0.0382 & 0.0016 & 0.0062 & 0.1604 & 0.6188 \\
0.2 & 0.0562 & 0.0613 & 0.0013 & 0.0050 & 0.2235 & 0.6474 \\
0.3 & 0.0831 & 0.0901 & 0.0032 & 0.0061 & 0.3552 & 0.7391 \\
0.4 & 0.1080 & 0.1128 & 0.0052 & 0.0091 & 0.6818 & 1.1574 \\
0.1 & 0.0287 & 0.0384 & 0.0030 & 0.0101 & 0.3782 & 1.4668 \\
0.2 & 0.0561 & 0.0654 & 0.0013 & 0.0036 & 0.2578 & 0.8136 \\
0.3 & 0.0841 & 0.0913 & 0.0039 & 0.0102 & 0.3724 & 1.1803 \\
0.4 & 0.1070 & 0.1198 & 0.0062 & 0.0115 & 0.5672 & 1.3293 \\
\hline
\end{tabular}

Table K.1b Experimental results during tracking straight line: gain set 2 . 


\section{Appendix K: Overview of the simulations and experiments}

\begin{tabular}{|c|ccccc|}
\hline \multicolumn{6}{|c|}{ Simulation table G.1 } \\
\multicolumn{5}{|c|}{ Simulation with meassurement noise and non linear damping: } & Table G.1 \\
\hline tun 1 & 882 & 1001005 & 150355 & 2007010 & \\
tun 2 & 682 & 1001005 & 150355 & 2007010 & \\
$\operatorname{tun} 3$ & 882 & 501005 & 150355 & 2007010 & \\
$\operatorname{tun} 4$ & 882 & 1001005 & 75355 & 2007010 & \\
$\operatorname{tun} 5$ & 882 & 1001005 & 150355 & 1007010 & \\
$\operatorname{tun} 6$ & 882 & 1001005 & 150355 & 2007010 & + \\
$\operatorname{tun} 7$ & 682 & 1001005 & 150355 & 2007010 & + \\
$\operatorname{tun} 8$ & 882 & 501005 & 150355 & 2007010 & + \\
$\operatorname{tun} 9$ & 882 & 1001005 & 75355 & 2007010 & + \\
$\operatorname{tun} 10$ & 882 & 1001005 & 150355 & 1007010 & + \\
$\operatorname{tun} 11$ & 882 & 501005 & 150355 & 2007010 & + \\
$\operatorname{tun} 12$ & 682 & 501005 & 150355 & 2007010 & + \\
$\operatorname{tun} 14$ & 882 & 501005 & 75355 & 2007010 & + \\
$\operatorname{tun} 15$ & 882 & 501005 & 150355 & 1007010 & + \\
$\operatorname{tun} 16$ & 1082 & 1001005 & 150355 & 2007010 & \\
$\operatorname{tun} 17$ & 1082 & 1001005 & 150355 & 2007010 & + \\
$\operatorname{tun} 18$ & 1082 & 501005 & 150355 & 2007010 & + \\
$\operatorname{tun} 20$ & 882 & 1501005 & 150355 & 2007010 & \\
$\operatorname{tun} 21$ & 882 & 1001005 & 200355 & 2007010 & \\
$\operatorname{tun} 22$ & 882 & 1001005 & 150355 & 2507010 & \\
$\operatorname{tun} 23$ & 882 & 1501005 & 150355 & 2007010 & + \\
$\operatorname{tun} 24$ & 882 & 1001005 & 200355 & 2007010 & + \\
$\operatorname{tun} 25$ & 882 & 1001005 & 150355 & 2507010 & + \\
\hline
\end{tabular}

Table L.1 Files used in table G.1. 


\begin{tabular}{|c|c|c|c|c|c|}
\hline \multicolumn{6}{|c|}{ Simulation with meassurement noise and non linear damping: tuning traject } \\
\hline & Lp1 & Lp2 & $\mathrm{Kp}$ & $\mathrm{Kd}$ & noise \\
\hline test 10 & 882 & 1001005 & 150355 & 2007010 & + \\
\hline test 11 & 882 & 1001005 & 150355 & 1007010 & + \\
\hline test 12 & 882 & 80805 & 150355 & 1007010 & + \\
\hline test 13 & 882 & 80805 & 1503510 & 1007010 & + \\
\hline test 14 & 882 & 80805 & 2003510 & 1007010 & + \\
\hline test 15 & 882 & 1001005 & 2007010 & 1007010 & + \\
\hline test 16 & 882 & 1001005 & 2008020 & 1007010 & + \\
\hline test 17 & 882 & 1001005 & 1004010 & 50205 & + \\
\hline test 18 & 882 & 1001005 & 1004010 & 30105 & + \\
\hline test 19 & 882 & 1001005 & 1004020 & 20205 & + \\
\hline test 20 & 662 & 1001005 & 1004020 & 20205 & + \\
\hline test 21 & 882 & 1001005 & 502020 & 10102 & + \\
\hline test 22 & 882 & 1001005 & 37172 & 320.5 & + \\
\hline test 23 & 882 & 1001005 & 1004020 & 30205 & + \\
\hline test 24 & 882 & 1001005 & 1004020 & 30205 & + \\
\hline test 25 & 882 & 1001005 & 1204020 & 30205 & + \\
\hline test 26 & 882 & 1001005 & 1504020 & 30205 & + \\
\hline test 27 & 882 & 1001005 & 1756020 & 30205 & + \\
\hline test 28 & 882 & 1001005 & 1754020 & 30205 & + \\
\hline test 29 & 882 & 1001005 & 2008020 & 30205 & + \\
\hline test 30 & 882 & 1001005 & 2006020 & 30205 & + \\
\hline test 31 & 882 & 1001005 & 2506020 & 30205 & + \\
\hline test 32 & 882 & 1001005 & 25010020 & 30205 & + \\
\hline test 33 & 882 & 1001005 & 30015040 & 30205 & + \\
\hline test 34 & 882 & 1001005 & 30015020 & 30205 & + \\
\hline test 35 & 882 & 1001005 & 30010020 & 30205 & + \\
\hline test 36 & 882 & 1001005 & 35010020 & 30205 & + \\
\hline test 37 & 882 & 1001005 & 40010020 & 30205 & + \\
\hline test 38 & 882 & 1001005 & 40020020 & 30205 & + \\
\hline test 39 & 882 & 1001005 & 40015020 & 30205 & + \\
\hline test 40 & 882 & 1001005 & 40010030 & 30205 & + \\
\hline \multicolumn{6}{|c|}{ Simulation with meassurement noise and non linear damping: tuning traject } \\
\hline pos 1 & 882 & 1001005 & 150355 & 2007010 & + \\
\hline pos 2 & 882 & 101010 & 35155 & 704010 & + \\
\hline
\end{tabular}

Table L.2 Files used for tuning system. 


\begin{tabular}{|c|c|c|c|c|c|c|c|c|}
\hline \multicolumn{9}{|c|}{ Experiments: gain set 1} \\
\hline & trajectory & velocity & Waves & $\exp 130$ & line & 0.1 & & \\
\hline $\exp 100$ & path & 0.2 & & $\exp 131$ & line & 0.2 & & \\
\hline $\exp 101$ & path & 0.1 & & $\exp 132$ & line & 0.3 & & \\
\hline $\exp 102$ & path & 0.3 & & $\exp 133$ & line & 0.4 & & \\
\hline $\exp 103$ & path & 0.4 & 0.01 & $\exp 134$ & line & 0.1 & 0.01 & \\
\hline $\exp 104$ & path & 0.1 & 0.01 & $\exp 135$ & line & 0.2 & 0.01 & \\
\hline $\exp 105$ & path & 0.3 & 0.01 & $\exp 136$ & line & 0.3 & 0.01 & \\
\hline $\exp 106$ & path & 0.4 & 0.01 & $\exp 137$ & line & 0.4 & 0.01 & \\
\hline $\exp 158$ & position & & & & & & & \\
\hline $\exp 159$ & position & & 0.01 & $\exp 138$ & line & 0.2 & & init. error \\
\hline \multirow[t]{7}{*}{$\exp 160$} & position & & 0.02 & $\exp 139$ & line & 0.2 & 0.01 & init. error \\
\hline & & & & $\exp 140$ & line & 0.2 & & init. error \\
\hline & & & & $\exp 141$ & line & 0.2 & 0.01 & init. error \\
\hline & & & & $\exp 148$ & path & 0.2 & & init. error \\
\hline & & & & $\exp 149$ & path & 0.2 & 0.01 & init. error \\
\hline & & & & $\exp 150$ & path & 0.2 & & init. error \\
\hline & & & & $\exp 151$ & path & 0.2 & 0.01 & init. error \\
\hline \multicolumn{9}{|c|}{ Experiments: gain set 3} \\
\hline $\exp 108$ & path & 0.1 & & exp122 & line & 0.1 & & \\
\hline $\exp 109$ & path & 0.2 & & $\exp 123$ & line & 0.2 & & \\
\hline $\exp 110$ & path & 0.3 & & $\exp 124$ & line & 0.3 & & \\
\hline $\exp 111$ & path & 0.4 & & $\exp 125$ & line & 0.4 & & \\
\hline $\exp 112$ & path & 0.1 & 0.01 & $\exp 126$ & line & 0.1 & 0.01 & \\
\hline $\exp 113$ & path & 0.2 & 0.01 & $\exp 127$ & line & 0.2 & 0.01 & \\
\hline $\exp 114$ & path & 0.3 & 0.01 & exp128 & line & 0.3 & 0.01 & \\
\hline $\exp 115$ & path & 0.4 & 0.01 & exp129 & line & 0.4 & 0.01 & \\
\hline $\exp 116$ & position & & & & & & & \\
\hline $\exp 117$ & position & & 0.01 & $\exp 153$ & path & 0.2 & & init. error \\
\hline \multirow[t]{4}{*}{$\exp 118$} & position & & 0.02 & $\exp 154$ & path & 0.2 & 0.01 & init. error \\
\hline & & & & $\exp 155$ & path & 0.2 & & init. error \\
\hline & & & & $\exp 156$ & line & 0.2 & & init. error \\
\hline & & & & $\exp 157$ & line & 0.2 & 0.01 & init. error \\
\hline \multicolumn{9}{|c|}{ Experiments: gain set 2} \\
\hline $\exp 119$ & position & & & & & & & \\
\hline $\exp 120$ & position & & 0.02 & & & & & \\
\hline $\exp 121$ & position & & 0.01 & & & & & \\
\hline
\end{tabular}

Table L.3 Files of the experiments used in the different tables and figures. 


$\begin{array}{cl}\text { Column } & \text { Explanation: } \\ 1 & \text { measured x position supply ship } \\ 2 & \text { measured y position supply ship } \\ 3 & \text { measured z position supply ship } \\ 4 & \text { measured } \phi \text { position supply ship } \\ 5 & \text { measured } \vartheta \text { position supply ship } \\ 6 & \text { measured } \varphi \text { position supply ship } \\ 7 & x \text { position main ship } \\ 8 & y \text { position main ship } \\ 9 & \varphi \text { position main ship } \\ 10 & \dot{x} \text { velocity main ship } \\ 11 & \dot{y} \text { velocity main ship } \\ 12 & \dot{\varphi} \text { velocity main ship } \\ 13 & \ddot{x} \text { acceleration main ship } \\ 14 & \ddot{y} \text { acceleration main ship } \\ 15 & \ddot{\varphi} \text { acceleration main ship } \\ 16 & x \text { observed position desired trajectory } \\ 17 & y \text { observed position desired trajectory } \\ 18 & \varphi \text { observed position desired trajectory } \\ 19 & \dot{x} \text { observed velocity desired trajectory } \\ 20 & \dot{y} \text { observed velocity desired trajectory } \\ 21 & \dot{\varphi} \text { observed velocity desired trajectory } \\ 22 & \ddot{x} \text { observed acceleration desired trajectory } \\ 23 & \ddot{y} \text { observed acceleration desired trajectory } \\ 24 & \ddot{\varphi} \text { observed acceleration desired trajectory } \\ 25 & x \text { observed position supply ship } \\ 26 & y \text { observed position supply ship } \\ 27 & \varphi \text { observed position supply ship } \\ 28 & \dot{x} \text { observed velocity supply ship } \\ 29 & \dot{y} \text { observed velocity supply ship } \\ 30 & \dot{\varphi} \text { observed velocity supply ship } \\ 31 & \ddot{x} \text { observed acceleration supply ship } \\ 32 & \ddot{y} \text { observed acceleration supply ship } \\ 33 & \ddot{\varphi} \text { observed acceleration supply ship } \\ 34 & x \text { observed position synchronization error } \\ 35 & y \text { observed position synchronization error } \\ 36 & \varphi \text { observed position synchronization error } \\ 37 & \dot{x} \text { observed velocity synchronization error } \\ 38 & \dot{y} \text { observed velocity synchronization error } \\ 39 & \dot{\varphi} \text { observed velocity synchronization error } \\ 40 & \ddot{x} \text { observed acceleration synchronization error } \\ 41 & \ddot{y} \text { observed acceleration synchronization error } \\ 42 & \ddot{\varphi} \text { observed acceleration synchronization error } \\ & \end{array}$


$x$ body fixed saturated control input $y$ body fixed saturated control input $\varphi$ body fixed saturated control input $x$ body fixed calculated control input $y$ body fixed calculated control input $\varphi$ body fixed calculated control input left thruster control input right thruster control input

51 tunnel thruster control input

52 left rudder control input

53 right rudder control input

54 time 


\section{Appendix L: Conference article}

This appendix includes the draft version of the article submitted to the IFAC conference on Contol Applications in Marine Systems 2004., CAMS 2004. 


\title{
EXPERIMENTAL RESULTS ON SYNCHRONIZATION CONTROL OF SHIP RENDEZVOUS OPERATIONS
}

\author{
Erik Kyrkjebø ${ }^{*}$ Michiel Wondergem ${ }^{* *}$ \\ Kristin Y. Pettersen ${ }^{*}$ Henk Nijmeijer ${ }^{* *}$ \\ * Department of Engineering Cybernetics, Norwegian University \\ of Science and Technology, Norway, E-mail: \\ Erik.Kyrkjebo@itk.ntnu.no,Kristin.Y.Pettersen@itk.ntnu.no \\ ${ }^{*}$ Department of Mechanical Engineering, Eindhoven \\ University of Technology, The Netherlands, \\ E-mail:M.Wondergem@student.tue.nl, H.Nijmeijer@tue.nl
}

\begin{abstract}
The paper presents experimental results on rendezvous control of ships using synchronization techniques. In particular, recent results on external synchronization for underway replenishment of ships are verified through a back-to-back comparison between experimental results and ideal simulations. The experiments illustrate that the synchronization controller yields exponential convergence of the closed-loop errors for position keeping, and uniform ultimate boundedness of the closed-loop errors during trajectory tracking. The gain tuning process is motivated, and the effects of measurement noise and environmental disturbances on the control scheme performance are discussed.
\end{abstract}

Keywords: Synchronization, Nonlinear control, Ship control, Observers, Tracking

\section{INTRODUCTION}

The first underway replenishment operation (UNREP) took place over a hundred years ago during an American blockade of Spanish warships outside of Cuba in 1899 (Miller and Combs, 1999), and since then much attention has been given to the problem of underway replenishment at sea for military ships to avoid or shorten port time. The control approaches of these rendezvous use flags and signals to communicate control commands between ships (FAS, 1999; NROTC, 2003), or some sort of tracking control of both ships in order to maintain trajectories that provide joint motion suitable for replenishment (Skjetne et al., 2003). Kyrkjeb $\varnothing$ and Pettersen (2003) proposed an external synchronization scheme to dynamically control the supply ship on the basis of the observed error in position between the two ships, as opposed to using trajectory tracking control where the two ships have separate tracking controllers with predefined trajecto- ries. In this paper, experimental results for this control scheme are presented.

Synchronization is found both as a natural phenomenon in nature as in the flashing of fireflies, choruses of crickets and musical dancing, as well as the controlled synchronization of a pacemaker or a transmitter-receiver system. Systems synchronize to each other in order to coordinate their operation, and the synchronization phenomenon was early reported by Huygens (1673) who observed that a pair of pendulum clocks hanging from a lightweight beam oscillated with the same frequency. Synchronization has in the last decade attracted an increasing interest from researchers within physics, dynamical systems, circuit theory, and more lately control theory. The theory of synchronization has been applied in control problems by e.g. Blekhman (1988), Nijmeijer (1997), Pogromsky and Nijmeijer (1998), Nijmeijer (2001), Rodriguez-Angeles and Nijmeijer (2001) and Nijmeijer and Rodriguez-Angeles (2003). In this paper we 
present experimental results on the external synchronization control for underway replenishment of ships as presented in Kyrkjebø and Pettersen (2003). This approach only requires that the dynamic model of the supply ship is known. It does not require that the dynamic model of the main ship is known. Furthermore, only position measurements of the two ships are needed for control. In particular, nonlinear observers are designed to estimate the velocities and accelerations of the two ships based on the position measurements and the supply ship model. The observercontroller scheme provides semi-globally exponentially stable error dynamics for position keeping, and semi-global uniform uitimate boundedness of the error dynamics during trajectory tracking.

In this paper, we investigate the advantages, shortcomings and the practical usefulness of the synchronization control scheme presented in Kyrkjeb $\emptyset$ and Pettersen (2003) using experimental data from tests with a model ship. Section 2 gives a short review of the synchronization observer-controller scheme, and in Section 3 the exponential convergence for position keeping, and ultimate boundedness for trajectory tracking are illustrated using both simulated results and experimental data from a model basin. The influence of wave disturbances, model errors, gain tuning and measurement noise on the control scheme are discussed in Section 4, while conclusions together with future work are presented in Section 5.

\section{SYNCHRONIZATION CONTROL SCHEME}

In this section we present the synchronization control scheme, which includes an error-observer and an observer for the supply ship states. While the results presented in Kyrkjeb $\emptyset$ and Pettersen (2003) are applicable to synchronizing any two marine vehicles (ship-ship, ship-AUV, AUV-AUV), we here focus our attention on the synchronization of two ships during ship rendezvous operations. The observer-controller scheme does not require information about the dynamic model of the main ship, only the dynamic model of the supply ship, and furthermore only requires position and attitude measurements for both ships. The supply ship is synchronized with the main ship through the control law, and is in fact a physical observer of the main ship states. The observer-controller scheme provides semi-global exponentially stable error dynamics for position keeping, and semi-global uniform ultimate boundedness of the error dynamics during trajectory tracking.

The general dynamic ship model in vectorial form (Fossen, 1994) can be written

$$
\begin{aligned}
\mathbf{M}_{i}\left(\eta_{i}\right) \ddot{\eta}_{i}+\mathbf{C}_{i}\left(\eta_{i}, \dot{\eta}_{i}\right) \dot{\eta}_{i}+ & \mathbf{D}_{i}\left(\eta_{i}, \dot{\eta}_{i}\right) \dot{\eta}_{i} \\
& +\mathbf{g}\left(\eta_{i}\right)=\tau_{i}
\end{aligned}
$$

where $i \in s, m$ denote the supply ship and the main ship respectively. The main ship is the replenished ship receiving cargo, while the supply ship is the replenishment vessel. The matrix $\mathbf{M}$ is the matrix of inertia and added mass, and $\mathbf{D}$ the damping matrix. The damping is here assumed to be linear. $\mathbf{C}$ is the Coriolis and Centripetal matrix also including added mass effects, and represented in terms of Christoffel symbols. The vector $\mathrm{g}$ represents gravitational/buoyancy forces and moments, while $\tau$ is the vector of control torques applied to the ship. The vector $\eta$ represents the Earthfixed position and orientation of the ship. For the experiments, $\eta$ is limited to the 3 DOF manoevring model form (Fossen, 1994) using $\eta=[x, y, \psi] \in \mathbf{R}^{2} \times$ $S O(1)$.

\subsection{Synchronization objective}

The objective is to synchronize a supply ship to the actual position of the main ship, in order to transfer fuel and supplies from one ship to another while the vessels are underway. This means that the supply ship is said to be synchronized to the main ship if its position/attitude and velocity coincide for all $t \geq 0$, or asymptotically for $t \rightarrow \infty$. Note that the position vector $\eta_{s}$ is synchronized to some offset constant reference $\eta_{r}$ to maintain a position alongside the main ship, and the problem is considered as synchronizing $\eta_{s}$ to $\eta_{m}$ by redefining $\eta_{m}=\eta_{m}^{\text {true }}-\eta_{r}$. For a trajectory with non-zero curvature, the forward velocity of the two ships must be different, and the redefinition implies that the velocity of the supply ship is synchronized to a computed main ship velocity in order to maintain the desired position in the inner or outer curve. The objective is thus to find a control law that stabilizes the error in position and velocity to zero; $(\mathbf{e}, \dot{\mathbf{e}})=(0,0)$.

\subsection{Synchronization rendezvous control}

2.2.1. The synchronization controller The synchronization controller $\tau_{s}$ will depend on estimated values for velocities and accelerations, and on measurements of position and attitude. The feedback control law is written as

$$
\begin{aligned}
\tau_{s} & =\mathbf{M}_{s}\left(\eta_{s}\right) \widehat{\ddot{\eta}}_{m}+\mathbf{C}_{s}\left(\eta_{s}, \widehat{\dot{\eta}}_{s}\right) \widehat{\dot{\eta}}_{m} \\
& +\mathbf{D}_{s}\left(\eta_{s}, \widehat{\dot{\eta}}_{s}\right) \hat{\dot{\eta}}_{m}+\mathbf{g}\left(\eta_{s}\right)-\mathbf{K}_{d} \widehat{\dot{\mathbf{e}}}-\mathbf{K}_{p} \mathbf{e} .
\end{aligned}
$$

where the synchronization errors are defined as

$$
\mathbf{e}=\eta_{s}-\eta_{m}, \quad \dot{\mathbf{e}}=\dot{\eta}_{s}-\dot{\eta}_{m}
$$

and $\mathbf{K}_{d}, \mathbf{K}_{p} \in \mathbf{R}^{n \times n}$ are positive definite symmetric gain matrices.

The control law utilizes the dynamic model of the supply ship, depending on the known supply position $\eta_{s}$ and observed supply velocity $\widehat{\eta}_{s}$, and the observed acceleration $\widehat{\ddot{\eta}}_{m}$ and velocity $\widehat{\dot{\eta}}_{m}$ for the main ship. Additional stabilizing proportional-derivative terms based upon the observed error in velocity $\hat{\dot{e}}$, and the known 
error in position $\mathbf{e}$, provides convergence and boundedness during replenishment. The supply ship uses the observed main ship states as reference states in the controller, and thus physically synchronizes itself to the main ship states. In effect, the supply ship becomes a physical observer of the main ship states.

Instead of using pre-calculated reference trajectories for both ships where tracking performance is vulnerable to waves, wind and currents, the reference for the supply ship is the main ship states. Hence, the performance of the operation is only dependent on the supply ship control accuracy, as opposed to the dependence of both control system when using precalculated trajectories.

2.2.2. The synchronization error observer The estimated values for the errors $e$ and $\dot{e}$ can be obtained through a full state nonlinear Luenberger observer

$$
\begin{aligned}
\frac{d}{d t} \widehat{\mathbf{e}} & =\widehat{\dot{\mathbf{e}}}+\mathbf{L}_{e 1} \widetilde{\mathbf{e}} \\
\frac{d}{d t} \widehat{\mathbf{e}} & =-\mathbf{M}_{s}\left(\eta_{s}\right)^{-1}\left[\mathbf{C}_{s}\left(\eta_{s}, \hat{\dot{\eta}}_{s}\right) \widehat{\dot{\mathbf{e}}}\right. \\
& \left.+\mathbf{D}_{s}\left(\eta_{s}, \hat{\dot{\eta}}_{s}\right) \widehat{\mathbf{e}}+\mathbf{K}_{d} \widehat{\dot{\mathbf{e}}}+\mathbf{K}_{p} \widehat{\mathbf{e}}\right]+\mathbf{L}_{e 2} \widetilde{\mathbf{e}}
\end{aligned}
$$

where $\mathbf{L}_{e 1}, \mathbf{L}_{e 2}$ are positive definite gain matrices, and the estimated position/attitude and velocity synchronization errors are defined as

$$
\widetilde{\mathbf{e}}=\mathbf{e}-\widehat{\mathbf{e}}, \quad \widetilde{\mathbf{e}}=\dot{\mathbf{e}}-\widehat{\dot{\mathbf{e}}}
$$

Note that the observers of Eqs. (4) and (5) introduces an extra correcting term in $\ddot{\mathbf{e}}=\widetilde{\dot{\mathbf{e}}}-\mathbf{L}_{e} \widetilde{\mathbf{e}}$ that yields faster performance during transients, but has some negative effects on noise sensitivity.

2.2.3. The supply ship state observer The estimated supply ship position and velocity values $\widehat{\eta}_{s}$ and $\widehat{\dot{\eta}}_{s}$ is found using the full state nonlinear observer

$$
\begin{aligned}
\frac{d}{d t} \widehat{\eta}_{s} & =\widehat{\dot{\eta}}_{s}+\mathbf{L}_{p 1} \widetilde{\eta}_{s} \\
\frac{d}{d t} \widehat{\dot{\eta}}_{s} & =-\mathbf{M}_{s}\left(\eta_{s}\right)^{-1}\left[\mathbf{C}_{s}\left(\eta_{s}, \widehat{\dot{\eta}}_{s}\right) \widehat{\mathbf{e}}\right. \\
& \left.+\mathbf{D}_{s}\left(\eta_{s}, \widehat{\hat{\dot{\eta}}}_{s}\right) \hat{\dot{\mathbf{e}}}+\mathbf{K}_{d} \widehat{\dot{\mathbf{e}}}+\mathbf{K}_{p} \mathbf{e}\right]+\mathbf{L}_{p 2} \widetilde{\eta}_{s}
\end{aligned}
$$

where $\mathbf{L}_{p 1}, \mathbf{L}_{p 2}$ are positive definite gain matrices, and the estimated supply ship position/attitude and velocity errors are defined as

$$
\widetilde{\eta}_{s}=\eta_{s}-\widehat{\eta}_{s}, \quad \widetilde{\dot{\eta}}_{s}=\dot{\eta}_{s}-\widehat{\dot{\eta}}_{s}
$$

2.2.4. The main ship state observer The estimated main ship velocity and acceleration values $\widehat{\dot{\eta}}_{m}$ and $\widehat{\ddot{\eta}}_{m}$ are not available through direct measurement, and must be reconstructed from the position/attitude and error estimates. To compensate for the lack of a dynamic model, the velocity and acceleration values for the main ship are reconstructed based on information of the supply ship and the synchronization closed-loop system. Estimates for $\eta_{m}, \dot{\eta}_{m}$ and $\ddot{\eta}_{m}$ are given as

$$
\begin{aligned}
\widehat{\eta}_{m} & =\widehat{\eta}_{s}-\widehat{\mathbf{e}} \\
\widehat{\dot{\eta}}_{m} & =\hat{\dot{\eta}}_{s}-\widehat{\dot{\mathbf{e}}} \\
\widehat{\ddot{\eta}}_{m} & =\frac{d}{d t}\left(\widehat{\dot{\eta}}_{s}-\widehat{\dot{\mathbf{e}}}\right) r \\
& =-\left(\mathbf{M}_{s}\left(\eta_{s}\right)^{-1}+\mathbf{L}_{e 2}\right) \tilde{\mathbf{e}}+\mathbf{L}_{p 2} \widetilde{\eta}_{s}
\end{aligned}
$$

where the last relation stems from (4) and (6).

The matrices $\mathbf{L}_{e 1}, \mathbf{L}_{e 2}, \mathbf{L}_{p 1}, \mathbf{L}_{p 2}$ are assumed positive definite, and chosen as symmetric matrices where $\mathbf{L}_{e 1}=\mathbf{L}_{p 1}$ and $\mathbf{L}_{e 2}=\mathbf{L}_{p 2}$ to simplify the stability analysis and tuning procedure.

\subsection{Stability}

The stability properties of the closed-loop error dynamics were investigated in Kyrkjeb $\varnothing$ and Pettersen (2003), and concluded with closed-loop errors being semi-global exponential convergent for position keeping, and semi-global uniformly ultimately bounded for trajectory tracking. The results are summarized in the following theorem:

Theorem 1. Consider the ship model (1), the controller (2) and the observers (4), (6) and (8). Under the assumption that the signals $\dot{\eta}_{m}(t)$ and $\ddot{\eta}_{m}(t)$ are bounded, i.e. that there exists bounds $\mathbf{V}_{M}$ and $\mathbf{A}_{M}$ such that

$$
\begin{aligned}
& \sup _{t}\left\|\dot{\eta}_{m}(t)\right\|=\mathbf{V}_{M}<\infty \\
& \sup _{t}\left\|\ddot{\eta}_{m}(t)\right\|=\mathbf{A}_{M}<\infty
\end{aligned}
$$

and that the minimum eigenvalues of the gain matrices $\mathbf{K}_{p}, \mathbf{K}_{d}, \mathbf{L}_{p 1}, \mathbf{L}_{p 2}$ are chosen to satisfy a set of lower bounds, then the synchronization closed-loop error

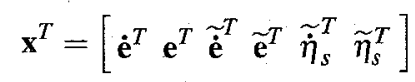

is semi-globally uniformly ultimately bounded when $\left(\dot{\eta}_{m}, \ddot{\eta}_{m}\right) \neq(\mathbf{0}, \mathbf{0})$. Furthermore, if the main ship achieve steady state $\left(\dot{\eta}_{m}, \ddot{\eta}_{m}\right)=(\mathbf{0 , 0})$ after $t_{s} \geq t_{0}$, we have semi-global exponential convergence of the synchronization closed-loop error $\mathbf{x}$ for $t \geq t_{s}$.

The bounds on the velocity and acceleration of the main ship can be established based on knowledge of the desired trajectory for the main ship during replenishment, or by the limitations imposed by the maximum acceleration and velocity given by the propulsion system. The boundedness assumption of the acceleration and velocity thus has a clear physical interpretation in marine control systems.

Furthermore, note that if position keeping during replenishment is considered, and the dynamic positioning system of the main ship is able to achieve steady state in finite time, then the control law of (2) and the observers (4), (6) and (8) yield semi-global exponential convergence of the synchronization closed-loop errors. 


\section{EXPERIMENTS}

Experiments were carried out to verify the theoretical results of the observer-controller scheme presented in Kyrkjeb $\emptyset$ and Pettersen (2003). In particular, the experiments aimed at obtaining an increased understanding of the proposed observer-controller scheme; its advantages and shortcomings. In order to investigate the differences between the theoretical results and practice, we present a back-to-back comparison of the experimental results with simulations under ideal conditions.

\subsection{Experimental Setup}

The experiments were carried out in the MCLab located at NTNU, Trondheim. The laboratory includes Cybership II, which is a Froude scaled (1:70) model supply vessel. The length of the ship is $1.3 \mathrm{~m}$ and the weight is about $24 \mathrm{~kg}$. Five actuators actuate the ship. At the stern there are two rpm-controlled screws with two rudders attached, while a two blade rpmcontrolled tunnel thruster is located at the bow. The maximum actuated forces are $2 \mathrm{~N}$ in surge, $1.5 \mathrm{~N}$ in sway and $1.5 \mathrm{Nm}$ in yaw.

A $300 \mathrm{MHz}$ on-board computer running QNX 6.2 on Cybership II controls the rudder stepmotors and thruster servomotors through an $\mathrm{H}$-bridge circuit. The Labview version 6.2 graphical user interface run under Windows XP 2002 on a Dell Latitude D800 with a $1.60 \mathrm{GHz}$ Intel Pentium $\mathrm{M}$ processor and $512 \mathrm{MB}$ RAM, and provides manual inputs, joystick and automatic control of the model ship. The control scheme is implemented in Matlab ver. 6.5.0 R13 with Simulink ver. 5.0, and generate make-files using OPAL-RT ver. 6.2. The position of the ship is measured with a 4 camera Proreflex motion capture system, 4 on-board active/passive responders and the NyPOS position measurement program running at $15 \mathrm{~Hz}$. The position measurement area was limited to $5 \mathrm{~m} \mathrm{x} 12 \mathrm{~m}$. Communication with the model ship is done through a wireless Breezecom network with a bandwidth of $2 \mathrm{Mbits} / \mathrm{s}$. The basin is equipped with a DHI wavemaker system. In the experiments, waves were generated using the JONSWAP distribution with a significant wave height of $0.01 \mathrm{~m}$ and a mean period of $\mathrm{Ts}=$ $0.75 \mathrm{~s}$.

The KPL thrust-allocation algorithm (Lindegaard, 2003) was used during the simulations and experiments. This thrust allocation can be divided into two parts: Force allocation, and inverse mapping. The force allocation distributes the computed control forces to the available actuators, while the inverse mapping finds the actuator set points that will actuate the desired force. The KPL thrust allocation is designed for a thruster controlled ship with rudders moving at low speed, and seeks to minimize the energy consumption.
Only one model ship is available at the lab, which necessitates the use of a virtual ship on a computer playing the role of the main ship. This virtual ship is based on a theoretical ship model, and is controlled using a back-stepping controller. The limitation of only one ship implies that there is no ship interaction during the experiments, and thus no observations nor influence from the Venturi-effect between the two ships. During the experiments with waves, only the supply ship experiences the waves as a disturbance, while the main ship sails in a virtual calm sea. In practical replenishment operations, both ships would pursue a heading into the waves, and the effect of environmental disturbances from currents, waves and wind would be similar on the two ships. The experiments with waves serves thus only as a measure of how robust the scheme is in regards to external disturbances, and not as an experiment on ship replenishment performance in waves. Furthermore, since the virtual main ship is a theoretical model running on a computer, modeling errors in the main ship model may affect the performance of the supply ship trying to physically synchronize to the main ship.

In the experiments, only the position of the supply vessel is measured, and thus it is difficult to verify the velocity synchronization directly. The only states available from the experimental data are therefore e, $\tilde{\mathbf{e}}$, $\tilde{\eta}_{m}$ and $\tilde{\eta}_{m}$. If the given experimental states compare with the corresponding states of the simulation results, it is plausible to think that the total state of the experimental results will compare to the simulated results as well.

\subsection{Simulation Setup}

The simulations serve as the ideal comparison case without modeling errors, and where no disturbances are present (no currents, wind or waves). The controller is based on a model without higher-order damping, and therefore only linear damping is included in the simulation model. The ship model is represented in the body frame as

$$
\begin{aligned}
& \mathbf{M}=\left[\begin{array}{ccc}
25.8 & 0 & 0 \\
0 & 33.8 & 1.0115 \\
0 & 1.0115 & 2.76
\end{array}\right] \\
& \mathbf{C}=\left[\begin{array}{ccc}
0 & 0 & -33.8 v-1.0115 r \\
0 & 0 & 25.8 u \\
33.8 v+1.0115 r-25.8 u & 0
\end{array}\right] \\
& \mathbf{D}=\left[\begin{array}{ccc}
0.72 & 0 & 0 \\
0 & 0.86 & -0.11 \\
0 & -0.11 & -0.5
\end{array}\right]
\end{aligned}
$$

\subsection{Tuning}

There is a duality in the gain tuning scheme on how to choose the $\mathbf{L}_{p 2}$ gain in the observers of Equations 
(4) and (6); a high gain yields good velocity estimates, but also introduces measurement noise to the observed velocity, which leads to highly fluctuating control actions. A low gain results in less accurate velocity estimates, but smoother control actions.

The $\mathbf{L}_{p 1}$ (Eq. (4) and (6)) should be kept low to minimize the influence of measurement noise. We do not have to choose between good estimates and low influence of measurement noise here (as we did when choosing $\mathbf{L}_{p 2}$ ), since we can lower the bounds on $\widetilde{\eta}_{\mathrm{s}}$ by increasing the $\mathbf{K}_{p}$ gain (Eq. 2). The $\mathbf{K}_{d}$ gain is then chosen to ensure that the region of attraction is large enough, and such that there is sufficient damping in the system to prevent oscillations during tracking. Thus, the semi-global validity of the scheme can be expanded by choosing the $\mathbf{K}_{d}$ gain larger. Note that the tuning of the controller is done in the body frame, where the gain matrices $\mathbf{K}_{p}$ and $\mathbf{K}_{d}$ are more intuitive, but are dependent on $\psi$.

\subsection{Exponential convergence}

To verify the theoretic results of global exponential convergence during dynamic positioning, the main ship was held at a constant position and heading in $\eta_{m}=[0,-1,0]^{T}$, while the supply ship was synchronized to a position alongside the main ship given by $\eta_{d}=[0,0,0]^{T}$. The initial state for the supply ship was chosen as $\eta_{s}=\left[-1,-1.5,-\frac{\pi}{2}\right]^{T}$ to illustrate the convergence in all states. The same gains were used in the experiments and simulations for the observer and controller to facilitate a back-to-back comparison, and were found empirically as

$$
\begin{aligned}
& \mathbf{K}_{p}=\operatorname{diag}\left[\begin{array}{lll}
35 & 15 & 5
\end{array}\right] \\
& \mathbf{K}_{d}=\operatorname{diag}\left[\begin{array}{lll}
70 & 40 & 10
\end{array}\right] \\
& \mathbf{L}_{p 1}=\operatorname{diag}\left[\begin{array}{lll}
8 & 8 & 2
\end{array}\right] \\
& \mathbf{L}_{p 2}=\operatorname{diag}\left[\begin{array}{lll}
10 & 10 & 10
\end{array}\right] .
\end{aligned}
$$

All errors are calculated and plotted in an earth-fixed North-East-Down frame. In Figure 1, the experimental results are presented with plots of the transient behavior and the steady-state. In Figure 2 the control errors are plotted, while Figure 3 shows the observer errors $\tilde{\mathbf{e}}, \tilde{\mathbf{e}}, \tilde{\eta}_{m}$ and $\tilde{\eta}_{m}$ during the simulation under ideal conditions.

The experimental results comply with the theoretical results of exponential convergence, and compare well with the simulated results. There is not much overshoot during positioning, which could otherwise have lead to dangerous situations when synchronizing to another marine structure, and this is furthermore an indication of stability margins with this set of gains for the observer-controller scheme. The settling time is sufficient for practical applications. The steady-state errors of the experiments show small persistent oscillations. These oscillations are not found in the simulations, and this suggests that the oscillatory behavior is
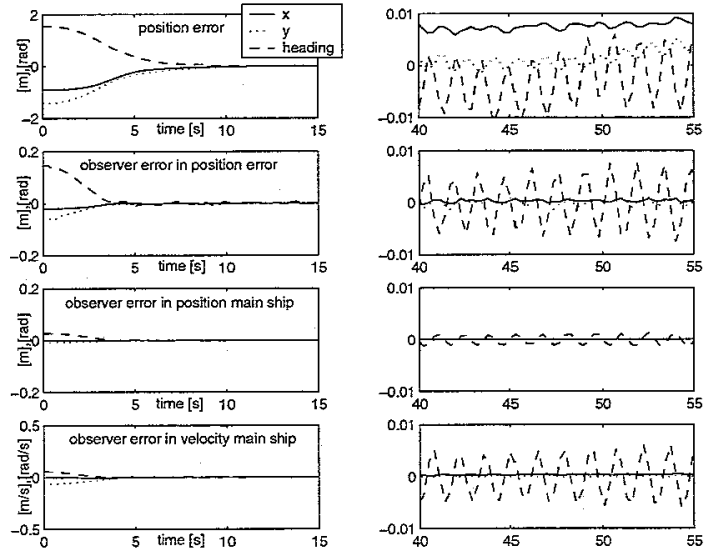

Fig. 1. Errors e, ẽ,$\tilde{\eta}_{m}$ and $\tilde{\dot{\eta}}_{m}$ during experiment
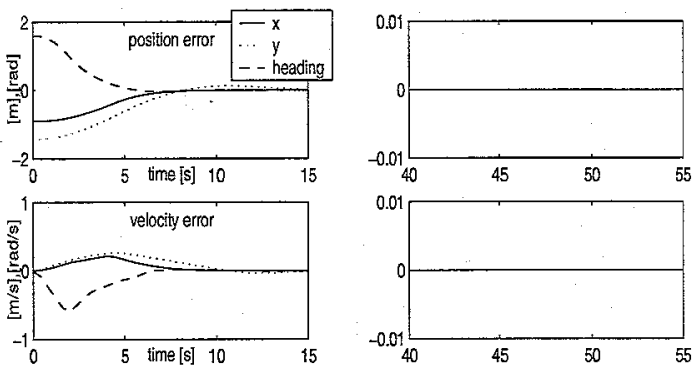

Fig. 2. Control errors e and è during simulation
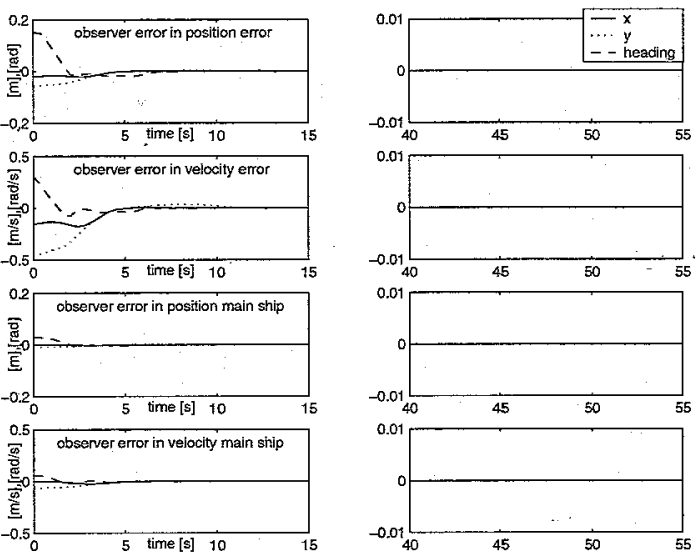

Fig. 3. Observer errors $\tilde{\mathbf{e}}, \tilde{\mathbf{e}}, \tilde{\eta}_{m}$ and $\tilde{\dot{\eta}}_{m}$ during simulation

caused by the measurement noise in the system, which influences the observer performance. The experiment was also carried out under wave disturbance, and the results of these experiments compare with the results presented here, with a slight increase in the errors as expected. In all, the experimental results for position keeping compare well with the simulations, and thus support the theoretical result of exponential convergence. 


\subsection{Ultimately boundedness}

The results of ultimate boundedness of the closedloop error during trajectory tracking were investigated using a trajectory with non-zero curvature. However, in practical applications the two ships would maintain a constant course and heading into the waves, and seek to keep the curvature of the trajectory at a minimum. A trajectory with non-zero curvature is illustrative of a situation where the replenishment ships are given greater manoevring freedom than in a straight line experiment, and would allow a replenishment operation to be performed in close waters. The results from the non-zero curvature experiments and simulations compare well with straight-line results for the observer-controller scheme. The non-zero curvature experiments shows the ability of the scheme to cope with changes in the main ship heading, which could otherwise lead to an aborted replenishment operation (FAS, 1999).

When the main ship trajectory has a non-zero curvature, the supply ship forward velocity and acceleration will differ from that of the main ship due to the curvature. The supply ship's relative forward velocity to the main ship will therefore depend on the distance between the two ships in a curve. When the supply ship sails the inner curve, the forward velocity of the supply ship is less than that of the main ship, and vice versa. An extreme case arises when the radius of the main ship curve is less than the distance between the two ships, where the supply ship in the inner curve would have to perform a backward movement.

The experiment shows the system behavior during trajectory tracking. The main ship tracks a predefined curved path with a desired velocity of $0.2 \mathrm{~m} / \mathrm{s}$, corresponding to a velocity of $3.5 \mathrm{knots}$ for the full scale ship. Initial states for the main were chosen as in Section 3.4, while the supply ship started in $\eta_{s}=\left[-1,1.5, \frac{\pi}{2}\right]^{T}$. The same gains were used in the experiments and simulations for the observer and controller to allow for a back-to-back comparison, and were found empirically as

$$
\begin{aligned}
& \mathbf{K}_{p}=\operatorname{diag}\left[\begin{array}{lll}
100 & 40 & 10
\end{array}\right] \\
& \mathbf{K}_{d}=\operatorname{diag}\left[\begin{array}{lll}
30 & 20 & 5
\end{array}\right] \\
& \mathbf{L}_{p 1}=\operatorname{diag}\left[\begin{array}{lll}
8 & 8 & 2
\end{array}\right] \\
& \mathbf{L}_{p 2}=\operatorname{diag}\left[\begin{array}{lll}
100 & 100 & 5
\end{array}\right] .
\end{aligned}
$$

In Figure 4 the $x y$-plot during this experiment is given. The errors during the experiment are plotted for transient behavior and steady-state in Figure 5. In Figure 6 , the control errors are shown in the body frame, while Figure 7 shows the observer errors $\tilde{e}, \tilde{e}, \tilde{\eta}_{m}$ and $\tilde{\eta}_{m}$ during the simulation under ideal conditions.

The experimental results comply with the theoretical results of ultimately boundedness of the closed-loop errors, and compare with the simulated results. Note that in the XY plot of Figure 4 and simulated results in Figs. 6 and 7, the experiments show better per-

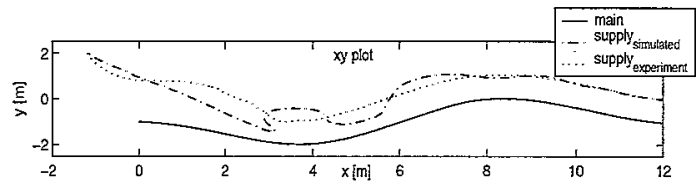

Fig. 4. $x y$-plot path
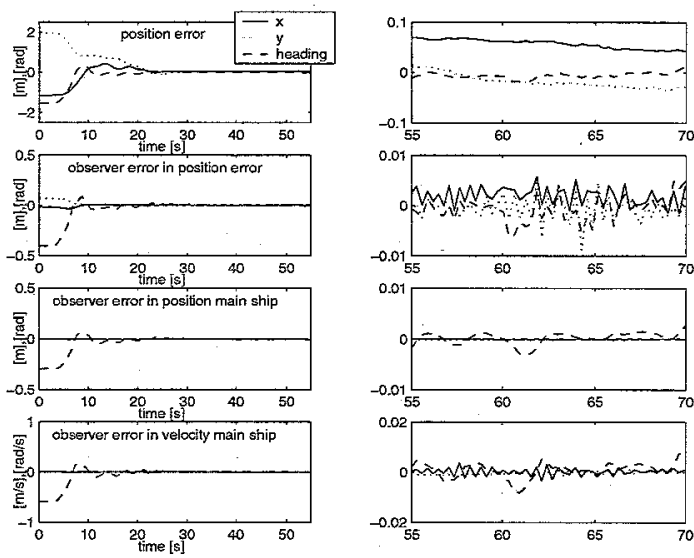

Fig. 5. Errors e, ẽ, $\tilde{\eta}_{m}$ and $\tilde{\eta}_{m}$ during experiment
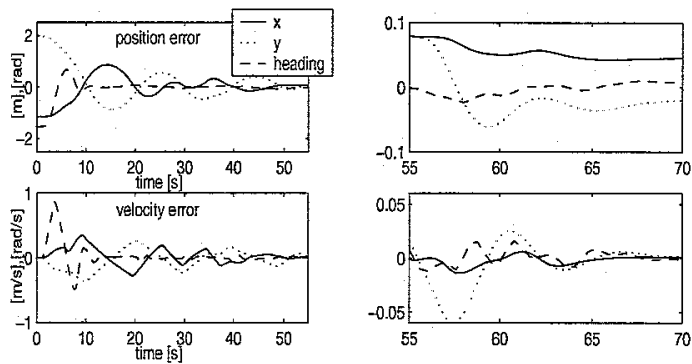

Fig. 6. Control errors e and $\dot{e}$ during simulation
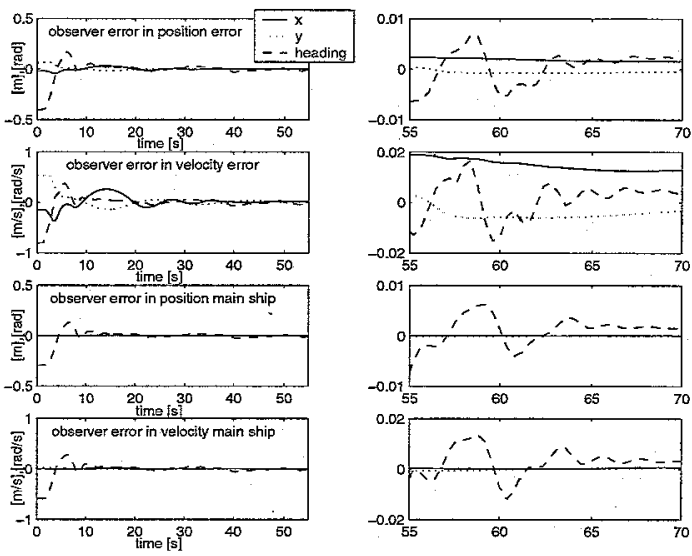

Fig. 7. Observer errors $\tilde{\mathbf{e}}, \tilde{\hat{\mathbf{e}}}, \tilde{\eta}_{m}$ and $\tilde{\eta}_{m}$ during simulation

formance than in the simulations. This is due to the fact that the ideal simulation model of Section 3.2 is restricted to linear damping. The non-linear damping inherent in the model ship is a stabilizing effect, and thus much less damping is needed in the controller gain $\mathbf{K}_{d}$. The back-to-back comparison is here shown 
using gains optimized for a model ship with nonlinear damping, and thus the performance in the linear simulations with these gains is not optimal. Similar performance as in the experiments can be shown in the simulations by increasing the $\mathbf{K}_{d}$ gain to compensate for the lack of non-linear damping.

There is a small bounded positive error in the $x$ position of the ship in the experiments (Fig. 5). Increasing $\mathbf{K}_{p}$ reduces the error, and contradicts the expected behavior if caused by a time delay in the system. The unexpected sign of the position error still remains an unresolved issue.

The observer accuracy diminishes slightly at the end of the path in Figure 5, which can be contributed to the reducing accuracy of the measurement system at the end of the basin. In all, the experimental results for trajectory tracking compare well with the simulations, and thus support the theoretical result of ultimately boundedness of the closed-loop errors. Note that empirical tuning of the observer-controller scheme is a particularly arduous task for trajectory tracking, and methodical gain tuning procedure should be adopted.

\subsection{Performance}

To assess the performance of the system, the timemean of the absolute error $\overline{\mathrm{E}}=\frac{1}{T} \int_{0}^{t}|\mathbf{e}| d t$ and the maximum of the absolute error $\mathrm{E}_{\max }$ are calculated under different conditions. The maximum error is presented to validate that there is no potential danger for collision during practical operations, and the mean error is presented to allow for easier comparison between the different results. Table 1 shows the different values during trajectory tracking in the experiments.

\begin{tabular}{|c|c|c|c|c|c|c|}
\hline \multicolumn{7}{|c|}{ Experiments:tracking predefined path } \\
\hline \multirow[b]{3}{*}{$\mathrm{ud}[\mathrm{m} / \mathrm{s}]$} & \multicolumn{6}{|c|}{ Without waves } \\
\hline & \multicolumn{2}{|c|}{ x_body $[\mathrm{m}]$} & \multicolumn{2}{|c|}{$y_{-}$body $[\mathrm{m}]$} & \multicolumn{2}{|c|}{ heading [deg] } \\
\hline & mean lel & $\max |e|$ & mean lel & $\max |e|$ & mean lel & $\max l e$ \\
\hline 0,1 & 0,0278 & 0,0421 & 0,0029 & 0,0128 & 0,4641 & 1,2490 \\
\hline 0,2 & 0,0548 & 0,0783 & 0,0123 & 0,0323 & 0,5214 & 2,4064 \\
\hline \multirow[t]{2}{*}{0,3} & 0,0790 & 0,1050 & 0,0367 & 0,0896 & 1,1860 & 3,7701 \\
\hline & \multicolumn{6}{|c|}{ With waves ( Johnswap: Hs $=0.01 \mathrm{~m}, \mathrm{Ts}=0.75 \mathrm{~s}$ ) } \\
\hline 0,1 & 0,0293 & 0,0503 & 0,0048 & 0,0169 & 0,4412 & 1,5126 \\
\hline 0,2 & 0,0555 & 0,0775 & 0,0146 & 0,0320 & 0,6818 & 2,2002 \\
\hline 0,3 & 0,0790 & 0,1047 & 0,0408 & 0,0969 & 1,0600 & 4,3774 \\
\hline
\end{tabular}

Table 1. Mean absolute error and maximum absolute error during tracking of the predefined path under different conditions.

The general trend in the mean absolute error and the maximum absolute error is that they increase with the speed. This is as expected, since if the ship tracks a distance with a higher velocity, it has relatively less time to compensate for the errors. The advantage of a higher speed is that the ship tracks the path smoother. The difference between the mean and maximum absolute error in the forward direction is relatively small, which indicates that the error is more or less constant. The results with and without waves show similar magnitude for the errors, which would indicate some robustness in the system. Note here, as presented in Section 3.1, that only the supply ship is subjected to the wave disturbance.

\section{DISCUSSION}

The experimental results for both position keeping and trajectory tracking complies with the theoretical results for the observer-controller scheme as presented in Kyrkjeb $\varnothing$ and Pettersen (2003). In addition, valuable lessons are learned from the experiments in regard to the gain tuning process, the influence of measurement noise and force saturation, model errors and robustness of the scheme.

Measurement noise influence the velocity observations in the observers (with large $\mathbf{L}_{p 2}=\mathbf{L}_{e 2}$ in Eqs. 4 and 6), and can lead to high commanded control actions. The observer performance is affected when the commanded forces are larger than the thruster limitations, since the commanded control forces and moments are used to progress the dynamical ship model in the observer. Here, the duality of the $\mathbf{L}_{p 2}$ gain is seen; a large gain may cause saturation in the forces, while a small gain may cause larger closed-loop errors. Furthermore, if there is a loss of measurements, the observer is forced to use previous states as inputs for a ballistic run, and thus the performance of the observers depend on both the quality of the ship model as well as the observer gains.

In the comparison of simulated and experimental results in Section 3.5, we can see a useful property inherent in the model ship; non-linear damping. Since the simulations are based on a linear damping model, the $\mathbf{K}_{d}$ gain must be increased in the simulations to compensate for this lack of inherent damping. If the tuning of the observer-controller scheme is based on simulations only, one should take care to include higherorder damping terms in the simulations to better model a real ship. The observer and controller gains $\mathbf{K}_{p}, \mathbf{K}_{d}$, $\mathbf{L}_{p 1}$ and $\mathbf{L}_{p 2}$ are optimized for either position keeping in Eq. 11 or trajectory tracking in Eq. 12 for better illustrations, but intermediate gains that perform well for both tasks can also be found. Empirical gain tuning of the observer-controller scheme is an arduous task due to the influence of observer performance on the controller performance and vice versa, and a methodical gain tuning procedure based on Nijmeijer and Rodriguez-Angeles (2003) or Section 3.3 should be adopted.

The robustness of the scheme is explored by introducing waves to the supply ship in the experiments. This does not affect the main ship, since it is a virtual ship running on a computer, and thus the results can be seen as the ability of the control scheme to suppress disturbances. The comparison between the experiments with and without disturbances is shown in Table 1, and the results show only small changes in performance 
when the supply ship is under the influence of waves. The robustness property is particularly useful during ship replenishment operations, where ships operating in close proximity of each other will influence each other (e.g. through Venturi-effects). Note that although the scheme is robust, it can not exceed the physical limitations of the ships. It can be seen that when the supply ship sails the outer curve with a velocity of $0.3 \mathrm{~m} / \mathrm{s}$ in Table 1 , the thrusters in the $y$-direction are saturated, and the errors increase.

\section{CONCLUSIONS AND FUTURE WORK}

Experimental results from tests with a model ship were presented, and aimed at exploring the advantages, shortcomings and practical usefulness of the synchronization based observer-controller scheme presented in Kyrkjeb $\emptyset$ and Pettersen (2003). A backto-back study was performed, where the experimental results were compared with ideal simulations of the theoretical results. The experimental results were found to comply well with the theoretical results of exponential convergence for position keeping and ultimate boundedness for trajectory tracking. The choice of controller and observer gains was motivated, and a review of the practical implications of measurement noise, model errors and disturbances was given. The experiments show that the synchronization observercontroller scheme is suited for practical replenishment operations. The scheme is robust with respect to environmental disturbances and force saturations, and suppresses the effects of model errors and measurement noise.

Future work aims at investigating the interaction effects on performance using two model ships, and to include higher order terms in the damping matrix of the observer-controller scheme to further explore the properties of non-linear damping.

\section{REFERENCES}

Blekhman, I.I. (1988). Synchronization in Science and Technology. ASME Press Translations. New York.

FAS (1999). Underway replenishment (UNREP) navy ships. [online] http://www.fas.org/man/dod101/sys/ship/unrep.htm. Rev: March 061999.

Fossen, T.I. (1994). Guidance and Control of Ocean Vehicles. John Wiley Ltd.

Huygens, C (1673). Horoloquium Oscilatorium. Paris, France.

Kyrkjebø, E. and K.Y. Pettersen (2003). Ship replenishment using synchronization control. Proc. 6th IFAC Conference on Manoeuvring and Control of Marine Craft pp. 286-291.

Lindegaard, Karl-Petter (2003). Acceleration Feedback in Dynamic Positioning. PhD thesis. Norwegian University of Science and Technology. Trondheim, Norway.
Miller, M.O. and J.A. Combs (1999). The next underway replenishment system. Naval Engineers Journal 111(2), 45-55.

Nijmeijer, H. (1997). On synchronization of chaotic systems. Proc. 36th Conf. Decision and Control pp. 384-388.

Nijmeijer, H. (2001). A dynamical control view on synchronization. Physica D 154 pp. 219-228.

Nijmeijer, H. and A. Rodriguez-Angeles (2003). Synchronization of Mechanical Systems. Vol. 46. World Scientific Series on Nonlinear Science, Series $\mathrm{A}$.

NROTC (2003). Underway replenishment. [online] http://www.unc.edu/depts/nrotc/classes/classinfo /NAVS52/12 Underway Replenishment.ppt. Rev: March 13, 2003.

Pogromsky, Yu.A. and H. Nijmeijer (1998). Observer based robust synchronization of dynamical systems. Int. J. Bifurc. Chaos 8 pp. 2243-2254.

Rodriguez-Angeles, A. and H. Nijmeijer (2001). Coordination of two robot manipulators based on position measurements only. International Journal of Control 74, 1311-1323.

Skjetne, R., I.-A. F. Ihle and T. I. Fossen (2003). Formation control by synchronizing multiple maneuvering systems. In: Proc. IFAC Conf. Manoeuvering and Control of Marine Crafts. IFAC. Girona, Spain. pp. 280-285. 